\title{
Probing small ribosomal subunit RNA helix 45 acetylation across eukaryotic evolution
}

Bortolin-Cavaillé Marie-Line ${ }^{(1)}$, Quillien Aurélie ${ }^{(1)(\#)}$, Thalalla Gamage Supuni ${ }^{(2)(\#)}$, Thomas. Justin M. ${ }^{(2)}$, Sas-Chen Aldema ${ }^{(4)(5)}$, Sharma Sunny ${ }^{(3)(6)}$, Plisson-Chastang Célia ${ }^{(1)}$, Vandel Laurence $^{(1)(7)}$, Blader Patrick ${ }^{(1)}$, Lafontaine Denis L.J. ${ }^{(3)}$, Schwartz Schraga ${ }^{(4)}$, Meier Jordan L. ${ }^{(2)}$ and Cavaillé Jérôme ${ }^{\left.(1){ }^{*}\right)}$

(1) Molecular, Cellular and Developmental Biology unit (MCD), Centre de Biologie Integrative (CBI), University of Toulouse; UPS; CNRS; 118 route de Narbonne, 31062 Toulouse, France

(2) Chemical Biology Laboratory, National Cancer Institute, Frederick, Maryland 21702, United States.

(3) RNA Molecular Biology, Fonds National de la Recherche Scientifique (FRS/FNRS), Université Libre de Bruxelles (ULB), B-6041 Gosselies, Belgium.

(4) Department of Molecular Genetics, Weizmann Institute of Science, Rehovot, Israel.

(5) Present address: The Shmunis School of Biomedicine and Cancer Research, The George S. Wise Faculty of Life Sciences, Tel Aviv University.

(6) Present address: Department of Cell Biology and Neurosciences, Rutgers, The State University of New Jersey, Piscataway, NJ-08904.

(7) Present address: Université Clermont Auvergne, CNRS, INSERM, iGReD, Clermont-Ferrand, France

$\left.{ }^{*}\right) \quad$ Corresponding author: Jerome.cavaille@univ-tlse3.fr; $\quad$ tel $\quad+33(0) 561335927$; fax: +33(0)561335886

(\#) equally contribution 


\section{Abstract}

NAT10 is an essential enzyme that catalyzes the formation of $N^{4}$-acetylcytidine $\left(\operatorname{ac}^{4} C\right)$ in eukaryotic transfer RNA (tRNA) and 18S ribosomal RNA (rRNA). Recent studies in human cells suggested that rRNA acetylation is dependent on SNORD13, a non-canonical box C/D small nucleolar RNA (SNORD) predicted to base-pair with 18S rRNA via two antisense elements. However, the selectivity of SNORD13-dependent cytidine acetylation and its relationship to NAT10's essential function in pre-rRNA processing remain to be defined. Here, we used CRISPR-Cas9 genome editing to formally demonstrate that SNORD13 is required for acetylation of a single cytidine residue of human and zebrafish $18 \mathrm{~S}$ rRNA. In-depth characterization revealed that SNORD13-dependent $\operatorname{ac}^{4} \mathrm{C}$ is dispensable for yeast or human cell growth, ribosome biogenesis, translation, and the development of multicellular metazoan model organisms. This loss of function analysis inspired a cross-evolutionary survey of the eukaryotic rRNA acetylation 'machinery' that led to the characterization of many novel SNORD13 genes in phylogenetically-distant metazoans and more deeply rooted photosynthetic organisms. This includes an atypical SNORD13-like RNA in D. melanogaster which appears to guide $\mathrm{ac}^{4} \mathrm{C}$ to $18 \mathrm{~S}$ rRNA helix 45 despite lacking one of the two rRNA antisense elements. Finally, we discover that $C$. elegans $18 \mathrm{~S}$ rRNA is not acetylated despite the presence of an essential NAT10 homolog. Altogether, our findings shed light on the molecular mechanisms underlying SNORD13-mediated rRNA acetylation across the eukaryotic tree of life and raise new questions regarding the biological function and evolutionary persistence of this highly conserved rRNA base modification. 


\section{Introduction}

N4-acetylcytidine $\left(\mathrm{ac}^{4} \mathrm{C}\right)$ in eukaryotic and archaeal rRNA was first documented in the 1970's, but the catalytic machinery and specific sites of this base modification have been defined only recently $(1,2)$. In eukaryotes, $\mathrm{ac}^{4} \mathrm{C}$ occurs at two positions within rRNA (helix 34 and 45) where it is catalyzed by the nucleolar GCN5-related RNA acetyltransferase NAT10, also referred to as Kre33 or Rra1 in yeast (3-5). In addition, recent studies showed that certain archaeal species harbour $>100 \mathrm{ac}^{4} \mathrm{C}$ residues in their rRNA, including at helix 45, placing cytidine acetylation on par with prevalent nucleoside modifications such as pseudouridines and 2'-O-methylations in these organisms $(6,7)$. Beside its involvement in rRNA modification, NAT10/Kre33 (assisted by its specific co-factor THUMPD1/Tan1) can modify C12 of serine and leucine tRNAs $(5,8)$ and has been suggested to modify eukaryotic mRNAs $(9,10)$. Orthogonal validation has, however, called into question the involvement of NAT10 in mRNA modification (6). In addition to modifying RNA, NAT10 may also be implicated in protein acetylation (11-13). Moreover, NAT10 is essential for pre-rRNA processing reactions leading to synthesis of 18S rRNA (5), and unsurprisingly it was associated with human diseases $(14,15)$. The importance of helix $45 \mathrm{ac}^{4} \mathrm{C}$ for cell homeostasis has not been addressed.

In yeast, the ability of Kre33 to specifically acetylate 18S rRNA was demonstrated to rely on two box C/D small nucleolar RNAs (SNORD), namely snR4 and snR45, which form imperfect base pairing interactions surrounding the targeted cytidine. Indeed, knockout of snR4 and snR45 led to the specific disappearance of 18S rRNA acetylation at SSU-C1280 (helix 34) and SSUC1773 (helix 45), respectively, while leaving tRNA acetylation unaffected (16). SNORDs are associated with four core proteins (SNU13, NOP56, NOP58, and Fibrillarin) and form nucleolar ribonucleoprotein (RNP) complexes known to mediate site-specific ribose methylation of rRNA, U6 spliceosomal snRNA and tRNAs; some of them are also involved in early nucleolar pre- 
rRNA processing (17). The possible contribution of snoRNA-associated proteins in rRNA acetylation remains undefined. Of note, SNORD13 (formerly U13) displays two rRNA complementarity tracts reminiscent of those present on snR45 (Figure 1), strongly implicating it as a human counterpart $(5,18)$. Consistent with this notion, inhibiting SNORD13 expression in a cancer cell line using antisense oligonucleotides causes a $\sim 50 \%$ decrease in levels of $\mathrm{ac}^{4} \mathrm{C}$ in 18S rRNA (5). However, the nucleotide-specificity of this inhibition and the residual level of acetylation did not afford to easily assess the impact of the modification on cell growth or ribosome biogenesis. Finally, three SNORD13-like RNA species were also reported in $A$. thaliana (snoR105, snoR108 and snoR146) but their roles in rRNA acetylation still awaits investigation (19).

Outside their potential to form two imperfect base-pairing interactions with $18 \mathrm{~S}$ rRNA, vertebrate SNORD13 and its S. cerevisiae and $A$. thaliana homologs are not highly conserved. This explains why, although SNORD13 was discovered thirty years ago $(18,20)$, its relationship with snR45 was only recognized recently (16). This suggests two distinct evolutionary hypotheses: either SNORD-mediated rRNA acetylation emerged early in a common ancestor of plants, fungi and animals and diverged thereafter, or alternatively, SNORD13 arose independently in these different lineages via convergent evolution. Irrespective of the scenario, the possibility also exists that SNORD13 has been lost in some lineages and, accordingly, helix 45 acetylation may rely on a stand-alone enzyme, or no longer be present in rRNA in certain species. This poses the question as to how SNORD-guided cytidine acetylation is conserved across the eukaryotic tree of life, and what cross-organismal commonalities in the nucleic acid acetylation machinery and its rRNA substrates govern this process.

Here, we use CRISPR-Cas9 genome editing to delete SNORD13 in human cells, confirming the hypothesis that this snoRNA is specifically required for acetylation of a single cytidine residue in 
small subunit ribosomal RNAs (SSU- $\mathrm{Cc}^{4} \mathrm{C} 1842$ located in rRNA helix45). Using this cell line and related model systems, we further establish that loss of SSU-ac ${ }^{4} \mathrm{C} 1842$ does not lead to obvious defects in terms of cell growth, protein synthesis, ribosome biogenesis, or the development of metazoan model organisms (zebrafish, flies). Finally, considering that Kre33/NAT10 is essential for pre-rRNA processing (5) while $\mathrm{ac}^{4} \mathrm{C}$ in helix 45 appears dispensable, we surveyed the conservation of the ribosomal RNA acetylation machinery across the eukaryotic tree of life. This cross-evolutionary analysis revealed that SNORD-mediated rRNA acetylation is widely conserved in Metazoans, but defined C. elegans as a multicellular organism in which rRNA acetylation has been lost. Our findings illuminate the molecular mechanisms underlying SNORD13-mediated rRNA acetylation across evolution and raise new questions regarding the role of highly conserved SSU-ac ${ }^{4} \mathrm{C} 1842$.

\section{Results}

Genetic deletion of human SNORD13 gene causes loss of SSU-ac ${ }^{4}$ C1842 - As a first step to probe directly the functional role of SNORD13 in rRNA modification, the human SNORD13 gene was inactivated in the haploid human chronic myelogenous leukemia-derived HAP1 cell line (21). In order to avoid potential transcriptional interference with the adjacent TTI2 gene, the 3'end region of SNORD13 was targeted for CRISPR-Cas9-mediated deletion (Figure 2A). PCR analysis identified three independent SNORD13-deficient clones (\#12/\#20/\#30) harboring the expected deletion (not shown), which rendered SNORD13 undetectable by Northern blot (Figure 2B). Three additional clones (\#13/\#19/\#22) were identified that were exposed to CRISPR/Cas9 without any deletion at SNORD13, providing a set of matched control cell lines subjected to identical clonal selection procedures and/or putative off-target effects. Unless otherwise specified, in subsequent experiments SNORD13-KO and WT are used to refer to a 
stoichiometric mixture of these three SNORD13-deficient and SNORD13-expressing clones, respectively.

To define the site-specific activity of SNORD13, we took a dual approach to analyze our mutant and WT cell lines. First, we directly measured $\mathrm{ac}^{4} \mathrm{C}$ levels in helix 45 using a mung bean nuclease protection assay coupled to reversed phase high performance liquid chromatography (RP-HPLC, (5)). Briefly, total RNA extracted from SNORD13-KO and WT cells were hybridized with an antisense DNA oligonucleotide overlapping helix 45 and its flanking sequences and were treated with mung bean nuclease. Protected rRNA fragments were then gel-purified and nucleoside hydrolysates obtained after nuclease P1 digestions were subjected to RP-HPLC analysis. As shown in Figure 2C, cytosine acetylation at helix 45 was completely abolished in SNORD13-KO cells as compared to WT. As orthogonal validation, RNA isolated from SNORD13-KO and WT cells was analyzed using $\operatorname{ac}^{4} \mathrm{C}$ sequencing chemistry (22). In this approach total RNA is treated with sodium cyanoborohydride under acidic conditions, causing chemical reduction of the acetylated residue. This reduced nucleobase causes misincorporation of non-cognate NTPs during reverse transcription (RT), enabling $\operatorname{ac}^{4} \mathrm{C}$ sites to be detected as Cto-T mutations upon cDNA sequencing. Applying this approach to the two cell lines, we detected SNORD13-dependent misincorporation at SSU- $\mathrm{ac}^{4} \mathrm{C} 1842$ but not at SSU-ac ${ }^{4} \mathrm{C} 1337$ (Figure 2D). These experiments validated our knockout strategy and unambiguously defined SNORD13 as a human counterpart of yeast snR45 required for guiding formation of $\mathrm{ac}^{4} \mathrm{C}$ at the terminal stem-loop of human 18S rRNA.

\section{SNORD13 deletion does not disrupt cell growth, pre-rRNA processing or protein} synthesis in human cells - Next, we assessed the phenotypic consequences of SNORD13 deletion and/or SSU-ac ${ }^{4} \mathrm{C} 1842$ loss. Analysis of proliferation by cell counting revealed that loss of SNORD13 did not impact cell growth significantly (Figure 2E) and that cell death rates and 
modalities were within normal range (Figure $2 \mathrm{~F}$ ). We next examined the levels of pre-rRNA precursors and mature rRNAs in SNORD13-KO cells by Northern blot (supplementary Figure 2A-B), RNase $\mathrm{H}$ mapping (supplementary Figure 2C) and pulse chase experiments (supplementary Figure 2D). These analyses revealed that loss of SNORD13 and/or SSU$\mathrm{ac}^{4} \mathrm{C} 1842$ does not interfere with normal pre-rRNA processing. An involvement of SNORD13 and/or SSU-ac ${ }^{4} \mathrm{C} 1842$ on ribosomal subunit assembly and global translation was also investigated by performing polysomal analysis. In this assay, whole cytoplasmic extracts from SNORD13-KO and WT cells were subjected to sucrose density gradient fractionation (10-50\%). As shown in supplementary figure $2 \mathrm{E}$, the distribution and amplitude of peaks corresponding to $40 \mathrm{~S}, 60 \mathrm{~S}, 80 \mathrm{~S}$ monosomes and polysomes were virtually identical in SNORD13-KO and WT cells. Moreover, detection of nascent protein production by puromycin labelling (SUnSET assay) revealed identical, translation-dependent incorporation patterns in SNORD13-KO and WT cells, again indicating that overall protein synthesis is unlikely to be severely impaired in SNORD13deficient cells (Figure 2G). To test whether SSU-ac ${ }^{4} \mathrm{C} 1782$ plays a more subtle role in finetuning aspects of ribosome function such as translational fidelity, we transfected WT and SNORD13-KO cells with firefly luciferase reporter genes bearing in-frame stop codons (UAA, UAG) or point mutations (R218S) that abrogate enzymatic luciferase activity. These reporters enable any decrease in translational accuracy to be detected via a corresponding increase in firefly luciferase activity. Our data indicate that stop codon read-through and amino acid misincorporation in SNORD13-KO cells was similar between WT and KO cells (Figure 2H-left panel). Finally, when using a set of Renilla-IRES-Firefly bicistronic vectors, we also showed that the efficiency of internal ribosome entry site (IRES)-mediated translation was apparently unaffected in SNORD13-KO cells (Figure 2H-right panel). Overall, these findings suggest that SNORD13 and SSU-ac ${ }^{4}$ C1842 are not essential for ribosome biogenesis or function; at least in standard laboratory growth conditions. 
SNORD13-deficient zebrafish embryos develop normally - In order to understand the biological relevance of SNORD13 at the whole organism level, a 189 bp-long, CRISPR-Cas9 mediated deletion overlapping the Danio rerio (zebrafish) snord13 gene locus on chromosome 8 was generated (Figure $3 \mathrm{~A}$ ). Crossing of individual heterozygotes led to the generation of homozygous adult snord13 knockout fish of normal appearance (not shown). To exclude any maternal contribution, three different homozygous females were crossed with heterozygous males. As expected, snord13 and $\mathrm{ac}^{4} \mathrm{C}$ in helix 45 were no longer detectable in RNA isolated from homozygous knockout fish, as judged by Northern blotting (Figure 3B) and $\mathrm{ac}^{4} \mathrm{C}$ sequencing (Figure 3C), respectively. Homozygous snord13 individuals in the progeny did not present any apparent morphological defects at 30 hours post fertilization (30 hpf) and developed normally until adulthood (Supplementary Figure 3A). To explore the possible occurrence of more subtle defects, such as activation of the nucleolar stress pathway in response to impaired ribosome biogenesis (23), we performed immunostaining against activated Caspase-3. No increase in apoptotic cells was observed in the developing brain (Figure 3D-E) or spinal cord (Figure 3F-G) of snord13-deficient embryos, as compared to WT. Accordingly, the level of expression of the pro-apoptotic bc/2, bax and puma genes, as judged by RT-qPCR, was not significantly altered in snord13 mutant embryos (supplementary Figure 3B). Finally, we performed immunostaining in WT and mutant embryos for the pan neural marker HuC/D which labels newly differentiated neurons in different structures including olfactory epithelium, pineal gland or optic tectum and the spinal cord. Again, no obvious difference could be detected between WT and mutant embryos (Figure 3D-G). Beside central nervous system development, we also sought to explore whether snord13 mutant displayed defects in another process such as vasculogenesis. We found that the vasculature of the trunk which is composed of the dorsal aorta (DA), the cardinal vein (CV) and intersegmental vessels was also normal in 30 hpf snord13-deficient embryos, as revealed by in situ hybridization against the key vascular transcription factor fli1a (Figures $\mathbf{3 H - I}$ ). Similarly, the expression levels of various markers of 
the neural system (Ihx1a, pax6a and otx5) and of the dorsal aorta trunk vasculature (cdh5 and fli1a) were unchanged between WT and snord13-KO embryos, validating the lack of phenotypes observed (Supplementary Figure 3B). In conclusion, neither SNORD13 nor $18 \mathrm{~S}$ rRNA $\mathrm{ac}^{4} \mathrm{C}$ in helix 45 appear to play any essential roles in embryonic zebrafish development.

\section{A cross-evolutionary survey of eukaryotic ribosomal RNA acetylation machinery - To}

more fully apprehend the evolutionary origin of SNORD-mediated rRNA acetylation, we revisited the phylogenetic distribution of SNORD13 throughout eukaryotes, focusing primarily on Metazoan (animals) and Archaeplastida (organisms capable of photosynthesis). Using BLAST searches with low stringency algorithm parameters together with manual inspection, we identified many novel candidate SNORD13 homologs outside the vertebrate lineage, including Urochordata, Cephalochordata, Echinodermata, Arthropoda, Mollusca, Brachiopoda, Cnidaria and even Porifera (Figure 4A/B). As depicted in Figure 4B, many of the newly-identified SNORD13 genes are located within introns, both in the sense or antisense orientation with respect to transcription of their host-gene transcript. In Archaeplastida, SNORD13 was also uncovered in phylogenetically distant land plants, including in the freshwater green alga $C$. braunii (Figure 4C). A full list of newly-identified SNORD13 and proposed snoRNA:rRNA duplexes is provided in Supplementary Data 1 and Supplementary Figure 1, respectively. These sequence analyses were accompanied by experimental profiling of $\mathrm{ac}^{4} \mathrm{C}$ at helix 45 in representative eukaryotic species. As listed in Figure 4A, acetylated cytidine was readily detected in the vast majority of organisms we examined, including unicellular eukaryotes such as slime molds ( $P$. polycephalum), ciliates (P. tetraurelia, $P$. falciparum) or microalgaes $(P$. tricornutum). We found, therefore, that SNORD13 is much more broadly distributed than initially thought and SNORD-mediated RNA acetylation is a widespread principle in eukaryotes. Consistent with this conclusion, NAT10 homologs were found in almost all organisms we queried, with the notable exception of E. gracilis (Figure 4A, Supplementary data 2). 
Atypical SNORD13-like homologs in Diptera - SNORD13-like genes are present in many groups of Arthropoda (Figure 5A, Supplementary data 1), including D. melanogaster and $B$. mori where they had previously been identified as SNORDs of unknown functions (named OrCD1 and Bm-10, respectively; $(24,25))$. In Diptera for which a genome annotation is available, Or-CD1 can be found either in large introns positioned in the 5'-UTR of two transcript isoforms generated from two alternative transcription sites (e.g. Swim, Tinagl1_2), or within short introns embedded in the open reading frame of the host-gene (e.g. Zfp15, Rps11) (Figure 5B). To our surprise, the published sequence of Or-CD1, as well as that of all newly-identified SNORD13like in Diptera, lack conserved antisense rRNA sequence at their 5'-end (Figure 5C). One potential explanation would be that the sequence upstream of the C-box is removed by exonucleolytic trimming of the spliced-out host-intron, very likely due to the absence of a 5'capped structure which normally plays a protective role against degradation (26). Although plausible for small introns, such as those in Zfp15 or Rps11 genes, this hypothesis cannot account for all observations. For example, many Or-CD1-like in Arthropoda are located in introns (Figure 4B), yet they still retain a conserved rRNA complementarity in their 5'-end segment (Figure 5C, Supplementary Figure 1). As an alternative, we reasoned that being intronic may not necessarily rule out independent transcription from distinct promoter regions. This is supported by the fact that SNORD13 can be found positioned either in the sense or antisense orientation relative to transcription of their host-gene (Figure 4B/5B), a scenario that is not compatible with post-transcriptional processing from introns.

To probe the mode of expression of Or-CD1 in Arthropoda, total RNAs extracted from D. melanogaster, L. humile (ants) and A. mellifera (bees) were subjected to immunoprecipitation with R1131 antibodies that recognize the 2,2,7 trimethylguanosine (TMG) cap structure, enabling its use as a proxy for independent transcription. Consistent with the expected size for small RNAs lacking 5'-end extension in D. melanogaster, Northern blot analyses detected a 
radioactive signal migrating at about 110 nucleotides (Figure 5D, see also Figure 5F). These RNA species were specifically immunoprecipitated with the R1131 antibodies indicating that, despite being located within an intron, Or-CD1 gene is independently transcribed. This conclusion is in line with prior studies showing that Or-CD1 represents one of the very few SNORD genes that recruit RNA polymerase II and the Little Elongation Complex (LEC;(27)). In agreement with phylogenetic comparison that identifies two conserved antisense rRNA elements (Figure 5C), Or-CD1 displays a 5'-capped extension in both L. humile and A. mellifera (Figure 5D), as evidenced by the sizes of RNAs recovered in the immunoprecipitation pellet fraction. This therefore identifies a second expression strategy by which independent transcription from introns can also yield "regular" extended forms of Or-CD1 (Figure 5E).

Thus, Or-CD1 in Diptera - but not in Arthropoda - displays only one antisense rRNA sequence (Figure 5C). In order to test if this remarkable fly SNORD13 was capable of guiding rRNA acetylation, a strain bearing a P-element inserted in the Or-CD1 gene was retrieved from the Bloomington Drosophila Stock Center (DmellPsnoRNA:Or-CD1G9117). After demonstrating that Or-CD1 was no longer detected in the mutant adult flies (Figure 5F), we showed that acetylation in helix45 was abolished (Figure 5G). Thus, SSU-C1968 in D. melanogaster can be targeted for acetylation by a SNORD13 homolog displaying a single antisense element. Of note, Or-CD1 adult knockout flies are viable, fertile and do not manifest gross abnormalities (not shown), thus strengthening the notion that N4-acetylcytidine at helix 45 is largely dispensable for viability, at least under standard laboratory conditions. Based on our findings, we propose to rename Or-CD1 as SNORD13.

\section{Life without $18 S$ rRNA acetylation: the cases of $C$. elegans and genetically modified} $\Delta \mathbf{s n R 4} \Delta \mathbf{s n R} 45$ mutant yeast. A key role in ribosome biology of $18 \mathrm{~S}$ rRNA helix 45 , which in mature small ribosomal subunits lies directly adjacent to the decoding site, is implied by its 
extreme sequence conservation in many phylogenetically-distant organisms belonging to all known eukaryotic supergroups. Furthermore, when mutations in helix 45 arise, they are often accompanied by compensatory base changes (Supplementary Figure 4A). The 5'-CCG-3' motif - wherein the second $\mathrm{C}$ is targeted for acetylation - was established as a key specificity sequence determinant for NAT10 $(3,6)$. Remarkably, a few well-studied organisms lack this conserved motif or even the targeted substrate cytidine, including flagellated protozoan parasites (T. vaginalis, T. brucei, L. major), protozoan algae (E. gracilis), ciliate protozoa ( $T$. thermophila), slime mold ( $P$. polycephalum), corn smut fungus ( $U$. maydis) or even the nematode (C. elegans) (Figure 4A). Other examples can be found in Supplementary Figure 4B. Only in the slime mold $P$. polycephalum did our ac4C sequencing reaction provide evidence for helix 45 acetylation within a non-consensus 5'-UCG-3' motif, suggesting that NAT10 may have a distinct biochemical specificity in this organism (Supplementary Figure 5). Of note, helix 34 of $P$. polycephalum is apparently not acetylated despite the presence of a similar 5'UCG-3'. Given its importance as animal model and the prior determination that it carries an essential NAT10 gene (named nath-10)(28), we next focused on C. elegans. As shown in Figure 6A-6B, helix 45 of C. elegans 18S rRNA harbors a 5'-CUG-3' motif also found in other nematodes, notably those belonging to the Clades IV and V. By definition, this motif variant that lacks a middle cytidine cannot be acetylated canonically. Nonetheless, since NAT10 is present in the worm genome, we verified the absence of any other $\operatorname{ac}^{4} \mathrm{C}$ residue within helix 45 (Figure 6C, not shown). More surprisingly, we found that the 5'-CCG-3' motif in helix 34 was also devoid of acetylation (Figure 6C). The lack of rRNA acetylation in C. elegans elsewhere than in helices 34 and 45 was further examined using anti-ac ${ }^{4} \mathrm{C}$ immuno-northern blotting, in which signals for acetylated RNA were found to be limited to tRNAs (Figure 6D). It is important to note that $\operatorname{ac}^{4} \mathrm{C}$ free ribosomes are not necessarily a universal feature of nematodes. Indeed, helix 45 was found to be acetylated in $S$. carpocapsae, an entomopathogenic nematode of the Steinernematidae family (Supplementary Figure 5). The evolutionary forces that drove 
disappearance of rRNA acetylation in C. elegans remain unknown. Nonetheless, our results indicate that the changes in robustness of vulval cell-fate specification observed when the essential nath10 gene was mutated $(28,29)$ did not reflect loss of rRNA modification but more likely the involvement of nath10 in pre-rRNA processing. A similar conclusion was reached recently when it was shown that the involvement of Fibrillarin in neural crest cell maturation depends on its role in pre-rRNA processing rather than in methylation (doi.org/10.1101/2021.11.25.469989). This is similar to prior observations in S. cerevisiae where Kre33 was shown to be essential but snR45 and/or SSU-ac ${ }^{4} \mathrm{C} 1773$ were not (16). As further validation of $\mathrm{ac}^{4} \mathrm{C}^{\prime} \mathrm{s}$ dispensability in rRNA we interrogated a double mutant yeast strain in which snR4 and snR45 genes were simultaneously deleted, producing rRNA completely devoid of $\mathrm{ac}^{4} \mathrm{C}$. As observed in Figure $6 \mathrm{E}$, growth of double $\Delta \mathrm{snR} 45 \Delta \mathrm{snR} 4$ mutants, as assayed in liquid or solid media, was undistinguishable from that of its wild-type counterpart whether at $30^{\circ} \mathrm{C}$ or higher sub-optimal temperatures. The lack of obvious phenotypes for $\Delta \mathrm{snR} 4 \Delta \mathrm{snR} 45$ mutants at $30^{\circ} \mathrm{C}$ was also previously reported (29). Collectively, these data are in agreement with previous findings showing that $\Delta \mathrm{Kre33}$ yeasts re-expressing a catalytic-dead form of Kre33 (H545A or R637A) grow normally, as compared to $\Delta$ Kre33 yeasts re-expressing a WT form of Kre33 (16). Thus, we confirm that the absence of rRNA acetylation is unlikely to contribute to the essential function of Kre33. This latter lies very likely with its modification-independent requirement for pre-rRNA processing events leading to the production of mature 18S rRNA (5). This conclusion does not, however, rule out some roles for tRNA acetylation, particularly under suboptimal growth conditions. Indeed, tan1 mutant strains show reduced growth rates at higher temperature $\left(39^{\circ} \mathrm{C}\right)$, presumably due to decreased stability of unmodified serine tRNAs (30). 


\section{Discussion}

In order to interrogate the function of a single SNORD13-dependent cytidine acetylation in helix 45 of $18 \mathrm{~S}$ rRNA, we have combined a cross-evolutionary survey of rRNA acetylation machinery across the eukaryotic tree of life and loss-of-function studies in four distinct models: budding yeasts, human cells, zebrafish and fly. This study represents one of the very few examples addressing the physiological roles of a SNORD in multicellular organisms bearing a germlinederived knockout (31-35). Although this peculiar rRNA $\operatorname{ac}^{4} \mathrm{C}$ is highly conserved, its loss surprisingly does not appear to grossly impact ribosome biogenesis or function, or even embryonic development. Consistent with this observation, we identify $C$. elegans as an evolutionary example of dispensable metazoan rRNA acetylation. Finally, the discovery of a 5' truncated form of SNORD13 in D. melanogaster suggests that the mechanism by which SNORDs assist NAT10 in catalyzing rRNA $a c^{4} \mathrm{C}$ is likely more diverse than previously anticipated and in some case may rely on a single antisense element.

An important question raised by our study concerns the specific role of $\mathrm{ac}^{4} \mathrm{C}$ in $18 \mathrm{~S}$ rRNA helix 45. In humans, the ribosomal protein eL41 (formerly RpL41) is positioned at the interface between the $40 \mathrm{~S}$ and $60 \mathrm{~S}$ subunits $(36,37)$ and directly contacts $\operatorname{ac}^{4} \mathrm{C} 1842$ (38). This led to the proposal that acetylation may mediate functionally important crosstalk between the two subunits (38). However, our experimental settings failed to uncover any robust defects in ribosomal subunit assembly or protein synthesis in human SNORD13-KO cells. This is perhaps not surprising since eL41 knockout in yeast does not affect translation unless formation of another inter-subunit contact is compromised (39). A prominent role of $\mathrm{ac}^{4} \mathrm{C}$ in eL41 assembly and/or RNA::protein interaction is also quite unlikely since eL41 is correctly incorporated into ribosomes of $T$. vaginalis (40), E. gracilis (41), L. donovani (42) and T. brucei (42), all of which lack acetylation in helix 45 (Supplementary Figure 6). Even more tellingly, the relative 
positioning of eL41 in ribosome and the 3-D architecture of helix 45 in the SSU of the archaea T. kodakarensis are not affected when TkNAT10 gene is deleted (6). Altogether, these observations point to a subtle molecular role for $\mathrm{ac}^{4} \mathrm{C}$, possibly in fine tuning ribosome structure and/or function in sub-optimal environmental contexts and/or sensitized genetic backgrounds. Unravelling the precise physiological relevance of $18 \mathrm{~S}$ RNA acetylation in multicellular organisms, if any, represents a conceptually and technically formidable, but important, task for future studies.

Our bioinformatics search has considerably expanded the inventory of SNORD13 sequences in metazoans, identifying homologs well beyond vertebrates where they were previously thought to be restricted. However, we were unable to identify SNORD13 counterparts in many major phyla including Hemichordata, Annelida, Nematoda, Fungi (other than budding yeasts), algae or unicellular eukaryotes. Although the most parsimonious hypothesis would be that rapidlyevolving SNORD13 sequences make them difficult to detect, one cannot exclude the possibility that SNORD13 may have been lost in these lineages, which would imply that in these organisms rRNA acetylation may be catalyzed by NAT10 alone. More sophisticated structural and biochemical analyses will be critical to solve this question. An additional related question is whether acetylation of helix 34 is also strictly dependent on an antisense SNORD, as shown only in S. cerevisiae to date (16). The characterization of RNA-guided rRNA acetylation systems in Archaea, should they exist, represents an additional avenue that may lend invaluable evolutionary and mechanistic insights into this process $(6,7)$.

Single nucleotide resolution cross-linking analysis has previously described how SNORDs guide the acetyltransferase to its rRNA substrate by use of two short base-pairing interactions on each side of the substrate cytosine, forming a "modification pocket" akin to H/ACA snoRNAs (SNORA) acting in pseudouridylation (16). During the course of this study, we unexpectedly 
identified independently-transcribed SNORD13 gene in D. melanogaster that produces RNA species lacking 5'-sequence complementary to rRNA (Figure 7). The same may also hold true in other Diptera for which SNORD13 is embedded within a very short intron (e.g. L. sericata, M. domestica). In this case, it is indeed more than likely that exonucleolytic processing of the hostintron produces uncapped RNAs with the C- and D-boxes brought together by a short terminal 5'-3' terminal stem, as commonly found for other intron-located SNORD (26). The genomic constraints and selection pressure that led to changes in SNORD13 expression strategies across evolution are unknown, yet they have inherent mechanistic repercussions since the deposition of $\mathrm{ac}^{4} \mathrm{C}$ in helix 45 in $D$. melanogaster does not strictly require two antisense elements flanking the rRNA $\mathrm{ac}^{4} \mathrm{C}$ site. This finding contrasts with conclusions drawn from mutagenesis of yeast snR45 where the 5' antisense element appears essential for acetylation (16). It therefore implies an alternative mode of action of SNORD13 in D. melanogaster and, also more broadly, in Diptera. Finally, the overall structure of SNORD13 in Diptera is indistinguishable from that of the canonical intron-located SNORDs which mediate rRNA ribose methylation. It thus raises the intriguing question as to whether other intronic SNORDs in these organisms, particularly those of still elusive function, might also mediate acetylation on still unidentified RNA targets.

Altogether, our data point to interlaced questions regarding SNORD13 sequence diversity across evolution, the molecular mechanisms underlying site-specific rRNA acetylation and the physiological importance of $\mathrm{ac}^{4} \mathrm{C}$ in rRNA in cell homeostasis, development and physiology. In our view, tackling these complex issues through an evolutionary perspective will be undoubtedly fruitful and may produce more surprises since, paradoxically not to mention ironically, observations made here with two widely-studied models - C. elegans and D. melanogaster depart considerably from those previously obtained from vertebrates and budding yeasts. 


\section{Materials and Methods}

Unless otherwise noted, all techniques for cloning and manipulating nucleic acids were performed according to standard protocols.

Generation and characterization of SNORD13-deficient HAP1 cells - HAP1 cells were grown at $37^{\circ} \mathrm{C}$ with $5 \% \mathrm{CO}_{2}$ in IMDM (Gibco; $4.5 \mathrm{~g} / \mathrm{L}$ glucose) supplemented with $10 \%$ fetal bovine serum (PAN biotech), $1 \mathrm{mM}$ Sodium Pyruvate (Gibco) and 1\% penicillin-streptomycin (Sigma-Aldrich). The human SNORD13 gene was disrupted via CRISPR/Cas9-mediated deletion. Two sgRNAs selected using the CRISPR design tool at https://zlab.bio/guide-designresources were cloned into pX459 V2.0 (Addgene \#62988) plasmid. HAP1 cells seeded in sixwell dishes (70-80\% confluency) were co-transfected using lipofectamine 2000 (Thermo Fisher Scientific) with pX459_sgRNA1 and pX459_sgRNA2 (500 ng each). 48 hours post-transfection, cells were transiently treated with puromycin $(2 \mu \mathrm{g} / \mathrm{mL} ; ~-36$ hours) before being subjected to clonal selection by limiting dilution. Deletion events were validated by PCR (primer sequences are listed in supplementary data S3) and three clones per genotype were randomly chosen for further analyses. The rate of cell growth was assayed using Beckman Coulter Z1 particle counter. Cells were seeded into a 24-well plate and counted in quadruplicate at each time points. Six independent proliferation assays were performed. Apoptosis was detected using Annexin V-FITC/propidium iodide staining according to the manufacturer's instructions (BioLegend). Cells were analyzed using FacsVerse (Becton Dickinson) with acquisition of 20,000 total events (BD FacsSuite). Two independent experiments were performed.

RNA extraction and rRNA analyses - Total RNA was prepared using TRI reagent (MRC) according to the manufacturer's instructions and treated with RQ1 RNAse-free DNase (Promega) and proteinase K (Sigma). For Northern blot analyses of low molecular-weight 
species, total RNA $(10 \mu \mathrm{g})$ was fractionated by electrophoresis on a $6 \%$ acrylamide, $7 \mathrm{M}$ urea denaturing gel and electrotransferred onto a Hybond N membrane (Amersham GE Helthcare) as described previously (43). For Northern blot analyses of higher molecular-weight species, 4 $\mu \mathrm{l}$ of total RNA $(2.5 \mu \mathrm{g} / \mu \mathrm{l})$ were added to $20 \mu \mathrm{l}$ of "glyoxal mix" (60\% DMSO, BTPE 1.2X, 5\% glycerol, 8\% glyoxal solution (Sigma) and $40 \mu \mathrm{g} / \mathrm{ml}$ Ethidium bromide), heat-denaturated at $55^{\circ} \mathrm{C}$ for $60 \mathrm{~min}$, chilled on ice for $10 \mathrm{~min}$ and separated by electrophoresis on a $1.2 \%$ agarose gel (Pipes 10 mM, Bis-tris 300 mM, EDTA $10 \mathrm{mM}$ ). Capillarity transfer was performed onto Hybond $\mathrm{N}+$ membrane. In all cases, transfer was followed by UV light irradiation and membranes were pre-hybridized for 1 hour in 5x SSC, 0.1\% SDS, 5x Denhardt's, $150 \mathrm{mg} / \mathrm{ml}$ yeast tRNA. Hybridization was carried out overnight at $50^{\circ} \mathrm{C}$ with $5^{\prime}-\left[{ }^{32} \mathrm{P}\right]$-labeled-DNA oligonucleotide probes $(100000 \mathrm{cpm} / \mathrm{ml})$. Membranes were washed twice with 0.1 XSSC, $0.1 \%$ SDS at room temperature (2x10 min) before autoradiography. Pulse chase experiments were performed as described in (44). The day before, 12-well plates are seeded with 500,000 cells. Cells are then incubated in methionine-free DMEM (Invitrogen) for 30 min at $37^{\circ} \mathrm{C}$, labeled for exactly 15 min with L-methyl ${ }^{3} \mathrm{H}$ methionine $(50 \mathrm{mCi} / \mathrm{ml})$ and rinsed twice with IMDM containing unlabeled methionine. They were then incubated for 0 min (rinsed immediately twice with cold PBS), $30 \mathrm{~min}, 1 \mathrm{~h}, 1 \mathrm{~h} 30,2 \mathrm{~h}, 2 \mathrm{~h} 30$ in regular IMDM. After the corresponding chase time, cells were rinsed twice with cold PBS and lysed with Tri-Reagent (MRC). RNA samples were glyoxal denatured and separated on a $1.2 \%$ agarose gel and passively transferred to a nylon membrane as described above. The membrane was exposed 5 weeks to Biomax KODAK MS films with a KODAK BioMax Transcreen LE. RNAse $\mathrm{H}$ mapping was performed as described in (44). A water solution containing $4 \mu \mathrm{g}$ of total RNA was heat-denatured $\left(95^{\circ} \mathrm{C}\right.$ for $\left.3 \mathrm{~min}\right)$ in the presence of an antisense oligonucleotide matching the 3'-end of 18S rRNA (RNase H_2, $100 \mu \mathrm{M})$. After allowing annealing by cooling down to room temperature, the RNA/oligonucleotide mixture was diluted to $30 \mu$ final with a reaction mix (1X RNase $\mathrm{H}$ reaction buffer, $65 \mu \mathrm{M}$ DTT, 0.5U/ $\mu$ l RNasin (Promega), $50 \mathrm{U}$ RNase H (New England Biolabs) and 
incubated at $37^{\circ} \mathrm{C}$ for $30 \mathrm{~min}$. The reaction was stopped by adding $0.3 \mathrm{M}$ sodium acetate $\mathrm{pH}$ 5.2/0.2 mM EDTA. RNA was phenol extracted, ethanol precipitated, loaded onto a 15\% acrylamide gel and analyzed by Northern blot ( ${ }^{32} \mathrm{P}$-labeled 3'18S oligo-probe) as described above. For primer extension, two $\mu \mathrm{g}$ of total RNA extracted from adult flies were mixed with a 5'${ }^{32}$ P-labeled oligo $(\sim 100000 \mathrm{cpm})$ and cDNA synthesis, generated according to the manufacturer's instructions, were resolved onto a $12 \%$ denaturing acrylamide gel.

Sucrose gradient sedimentation and Sunset assay - Exponentially growing WT and SNORD13-Ko cells ( 20 million per genotype) were treated with $100 \mu \mathrm{g} / \mathrm{ml}$ of cycloheximide (CHX, SIGMA) for $10 \mathrm{~min}$ at $37^{\circ} \mathrm{C}$, rinsed twice with cold $\mathrm{PBS} / \mathrm{CHX}(100 \mu \mathrm{g} / \mathrm{ml})$, collected by centrifugation (400 g, $5 \mathrm{~min}$ ) and resuspended in $5 \mathrm{ml}$ of cold Buffer A (10 mM Hepes, 1,5 mM $\mathrm{MgCl} 2,100 \mathrm{mM} \mathrm{KCl}, \mathrm{CHX} 100 \mu \mathrm{g} / \mathrm{ml}, 1 \mathrm{mM}$ DTT). Cells were then centrifuged (400g, $10 \mathrm{~min}$ at $4^{\circ} \mathrm{C}$ ), suspended in $700 \mu \mathrm{l}$ of Buffer $\mathrm{A}$ and incubated $10 \mathrm{~min}$ on ice. Cells were then mechanically disrupted with a cold Dounce homogenizer (B) and centrifuged for $10 \mathrm{~min}$ (1000 g at $4^{\circ} \mathrm{C}$ ). The cytoplasmic fraction (supernatant) was collected and centrifuged twice at $10000 \mathrm{~g}$ (10 $\mathrm{min}, 4^{\circ} \mathrm{C}$ ). About $1,5 \mathrm{mg}$ of protein was loaded on a $10-50 \%$ sucrose gradient and centrifuged for 2h45min at $39000 \mathrm{rpm}$ in an Optima L-100XP Ultracentrifuge (Beckman-Coulter) using a SW41 rotor (Beckman). Gradients were then monitored at $260 \mathrm{~nm}$ and fractions were collected from the top using a Foxy Jr. fraction collector (Teledyne ISCO). Sunset assay was performed as described in (45). Exponentially growing cells were treated with $1 \mu \mathrm{g} / \mathrm{ml}$ of puromycin for $20 \mathrm{~min}$. As a control, cycloheximide $(100 \mu \mathrm{g} / \mathrm{ml})$ was also added 15 min before puromycin treatment. Total cell extracts were then processed for western blot using an antipuromycine antibody (Millipore, clone 12D10 \#MABE343) as well as Anti-CDK9 (C12F7) mAb (cellsignal.com) as gel loading control. 
Immunoprecipitations - Thirty microliters of rabbit R1131 anti-trimethyguanosine (3 mg/ml, a gift of Dr. R. Luhrmann) were incubated with gentle agitation for $120 \mathrm{~min}$. at $4^{\circ} \mathrm{C} \_$with $80 \mu \mathrm{l}$ of Proteine A Sepharose ${ }^{\circ}$ 4B, Fast Flow from S. aureus (PAS, Sigma \#P9424) in $1 \mathrm{ml}$ of NET-150 buffer (50 mM Tris-HCl (pH 7.4), 150 mM NaCl, 0.05\% Igepal® CA-630, SIGMA). Mouse IgG (Jackson Immunoresearch \#315-005-008) was used as negative controls. PAS-Ig pellets were washed three times with $1 \mathrm{ml}$ of NET-150 buffer. Thirty micrograms of total RNAs was then added to PAS-R1131 (or PAS-IgG) in $0.5 \mathrm{ml}$ of NET-150 and incubated with gentle agitation for $60 \mathrm{~min}$. at $4{ }^{\circ} \mathrm{C}$. Pellets were then collected by centrifugation and washed seven times in $1 \mathrm{ml}$ of NET-150. RNAs from pellet and supernatant were extracted by SDS/phenol extraction and analyzed by Northern blot as described below.

Searching for SNORD13-like and 18S rRNA sequences in eukaryotic genomes - Using vertebrate SNORD13 sequences as queries, iterative BLAST searches with low stringency algorithm parameters, together with manual inspection of some hits, were conducted using eukaryotic genome databases found at https://blast.ncbi.nlm.nih.gov/Blast.cgi, https://rnacentral.org/, https://www.echinobase.org/entry/, http://mgbase.qnlm.ac/home, http://www.insect-genome.com/, https://bipaa.genouest.org/is/, https://i5k.nal.usda.gov/ webapp/blast/, https://spaces.facsci.ualberta.ca/ephybase/. Eukaryotic 18S rRNA sequences were retrieved either at https://www.arb-silva.de/ or https://www.ncbi.nlm.nih.gov/nucleotide/.

\section{Mung bean nuclease protection assay and RP-HPLC - Mung bean nuclease (MBN)} protection assay was performed exactly as described before (46). 500 pmol of the synthetic deoxy oligonucleotide (5'-TAATGATCCTTCCGCAGGTTCACCTACGGAAACCTTGTTACGAC TTTTAC-3') was incubated with 50 pmol of $18 \mathrm{~S}$ rRNA and $5 \%$ of DMSO in 0.3 volume of hybridization buffer (250 mM of HEPES, $500 \mathrm{mM}$ of $\mathrm{KCl}$ at $\mathrm{pH}$ 7). The mixture was incubated at $90^{\circ} \mathrm{C}$ for 5 minutes and then slowly cooled down to $45^{\circ} \mathrm{C}$ over $2 \mathrm{~h}$. After hybridization, 35 units 
of mung bean nuclease (New England Bio Labs (NEB)) and $0.02 \mathrm{mg} / \mathrm{ml}$ RNase A (SigmaAldrich) along with appropriate amount of 10x MBN buffer (NEB) were added to start digestion. The digestion was carried out at $35^{\circ} \mathrm{C}$ for $1 \mathrm{~h}$. The protected fragment (RNA-DNA hybrid) was extracted from the reaction mixture by phenol/chloroform extraction, followed by overnight ethanol precipitation. The protected rRNA fragment was separated from the complementary DNA oligonucleotides on a denaturing $7 \mathrm{M}$ Urea 15\% PAGE gel. Bands were visualized by ethidium bromide staining and the rRNA band was excised and eluted using the D-Tube TM Dialyzers according to the manufacturer's protocol for electro elution (Novagen). Eluted rRNA fragment was digested to nucleosides using P1 nuclease and alkaline phosphatase as described before (46). Nucleosides were next analyzed by RP-HPLC on a Supelcosil LC-18-S HPLC column $(25 \mathrm{~cm} \times 4.6 \mathrm{~mm}, 5 \mu \mathrm{m})$ equipped with a pre-column $(4.6 \times 20 \mathrm{~mm})$ at $30^{\circ} \mathrm{C}$ on an Agilent 1200 HPLC system, using a protocol described previously in (16).

Targeted ac4C sequencing - Nucleotide resolution sequencing of ac4C in 18S rRNA (helix 45 and helix 34) was performed as previously described (6). RNA samples were treated with sodium cyanoborohydride $\left(100 \mathrm{mM}\right.$ in $\left.\mathrm{H}_{2} \mathrm{O}\right)$ or vehicle $\left(\mathrm{H}_{2} \mathrm{O}\right)$ in a final reaction volume of 100 $\mu \mathrm{L}$. Reactions were initiated by addition of $1 \mathrm{M} \mathrm{HCl}$ to a final concentration of $100 \mathrm{mM}$ and incubated for 20 minutes at room temperature. Reactions were stopped by neutralizing the $\mathrm{pH}$ by the addition of $30 \mu \mathrm{L} 1 \mathrm{M}$ Tris- $\mathrm{HCl} \mathrm{pH}$ 8.0. After incubation, reactions were adjusted to 200 $\mu \mathrm{L}$ with $\mathrm{H}_{2} \mathrm{O}$, ethanol precipitated, desalted with $70 \%$ ice-cold ethanol, briefly dried on Speedvac, resuspended in $\mathrm{H}_{2} \mathrm{O}$, and quantified by using a Nanodrop 2000 spectrophotometer. Cellular total RNA from individual reactions (200 pg) was incubated with specific h45/h34 rev primer $(4.0 \mathrm{pmol})$ in a final volume of $20 \mu \mathrm{L}$. Individual reactions were heated to $65{ }^{\circ} \mathrm{C}$ for $5 \mathrm{~min}$ and transferred to ice for 3 min to facilitate annealing in SuperScript III reaction buffer (Invitrogen). After annealing, reverse transcriptions were performed by adding 5 mM DTT, 200 units SuperScript III RT, $500 \mu \mathrm{M}$ dNTPs (use $250 \mu \mathrm{M}$ dGTP) and incubating for 60 min at $55^{\circ} \mathrm{C}$. 
Reaction was quenched by increasing the temperature to $70{ }^{\circ} \mathrm{C}$ for 15 min and store at $4{ }^{\circ} \mathrm{C}$. cDNA $(2 \mu \mathrm{L})$ was used as template in $50 \mu \mathrm{L}$ PCR reaction with Phusion Hot start flex (New England Biolabs). Reaction conditions: 1X supplied HF buffer, 2.5 pmole each forward and reverse primer, $200 \mu \mathrm{M}$ each dNTPs, 2 units Phusion hot start enzyme, $2 \mu \mathrm{L}$ cDNA template. PCR products were run on a $2 \%$ agarose gel, stained with SYBR safe and visualized on UV transilluminator at $302 \mathrm{~nm}$. Bands of the desired size were excised from the gel and DNA extracted using QIA-quick gel extraction kit from Qiagen and submitted for Sanger sequencing (GeneWiz) using the forward PCR primer. Processed sequencing traces were viewed using 4Peaks software. Peak height for each base was measured and the percent misincorporation was determined using the equation: "Percent misincorporation $=($ peak intensity of $T) /($ sum of $C$ and T base peaks)`100\%".

Dual luciferase reporter assay - 100,000 WT and SNORD13-KO cells were seeded into a 24well plate for 24 hours. Cells were transfected using lipofectamine 2000 (Invitrogen) using 0.06 ng Renilla vector (internal control) and 500 ng of reporter Firefly vectors. 24 hours posttransfection, cells were harvested, lysed and luciferase activity was monitored using the DualLuciferase Reporter Assay kit (Promega) according to the manufacturer's recommendations. Luciferase detection was assayed on Centro LB 960 Microplate Luminometer (Mikrowin 2000 Software). Five-Six independent transfections were performed with each measurement made in triplicate.

Yeast cells and media conditions - S.cerevisiae cells deleted of snR4 and snR45 or wild-type cells were grown at $30^{\circ} \mathrm{C}$ in standard YEP medium (1\% yeast extract, $2 \%$ Bacto Peptone) supplemented with 2\% dextrose (YPD). For Liquid growth assay, yeast cells were inoculated from a fresh YPD-agar plate into liquid YPD and grown for $48-72$ hours at $30^{\circ} \mathrm{C}$ or $37^{\circ} \mathrm{C}$. Optical density at $600 \mathrm{~nm}\left(\mathrm{OD}_{600}\right)$ of cells was then measured and used to dilute cells into a 96 well 
plate (Cat \# 167008, Thermo Scientific) at equal ODs of $\sim 0.02 \mathrm{OD}\left(\mathrm{n}=3\right.$ or 12 for $30^{\circ} \mathrm{C}$ and $37^{\circ} \mathrm{C}$ samples, respectively). Cells were then grown for 90 hours in an Epoch 2 microplate spectrophotometer (BID EPOCH2 Microplate Spectrometer from BioTek) at $30^{\circ} \mathrm{C}$ and $37^{\circ} \mathrm{C}$ and OD was measured every 30 minutes. Measured $O D$ values were analyzed in $\mathrm{R}^{2}$. For presentation of growth curves, a regression line representing the mean of biological replicates was calculated using a generalized additive model. For Spot assay, yeast cells were inoculated from a fresh YPD-agar plate into liquid YPD and grown for 72 hours at $30^{\circ} \mathrm{C}$ and $37^{\circ} \mathrm{C}$. Optical density at $600 \mathrm{~nm}\left(\mathrm{OD}_{600}\right)$ was subsequently measured and cells were serially diluted in $2 \%$ YPD, such that OD of cells varied from 0.02 OD to 0.00016 OD (5 fold dilutions). $3 \mu$ of each diluted sample was used to spot cells on a YPD-agar plate, which was transferred to $30^{\circ} \mathrm{C}$ and $37^{\circ} \mathrm{C}$. After 48 hours of incubation pictures were taken of the colonies. Each strain was grown in duplicates.

Ethics statement and Zebrafish care - Fish were handled in a facility certified by the French Ministry of Agriculture (approval number A3155510). The project has received an agreement number APAFIS\#7124-20161 00517263944 v3. Anesthesia and euthanasia procedures were performed in Tricaine Methanesulfonate (MS222) solutions as recommended for zebrafish (0.16 $\mathrm{mg} / \mathrm{ml}$ for anesthesia, $0.30 \mathrm{mg} / \mathrm{ml}$ for euthanasia). All efforts were made to minimize the number of animals used and their suffering, in accordance with the guidelines from the European directive on the protection of animals used for scientific purposes (2010/63/UE) and the guiding principles from the French Decret 2013-118. Embryos were raised and staged according to standard protocols and the Recommended Guidelines for Zebrafish Husbandry Conditions $(47,48)$

Generation of Zebrafish snord13 mutants - The two guide RNAs (gRNA) were designed using CHOPCHOP CRISPR Design website (http://chopchop.cbu.uib.no). The designed oligos 
were annealed and ligated into the gRNA plasmid pX459 digested by BbsI (Thermo Scientific). The gRNAs were generated using the MEGAshortscript T7 transcription kit (Ambion) with PCR fragments containing T7 promoter. Transcripts were purified by phenol-chloroform extraction

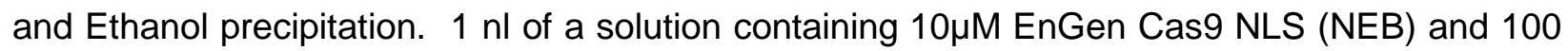
$\mathrm{ng} / \mu \mathrm{l}$ of gRNAs was injected at the one-cell stage. WT, heterozygous, and homozygous SNORD13 animals were then identified by PCR. Sequence primers can be found in (supplementary data S3).

RNA extraction, Reverse transcription and real-time PCR - Total RNAs from 15 wild type and 15 mutant zebrafish embryos were extracted using TRI reagent (MRC) and treated with RQ1-RNase free DNase I (Promega) and Proteinase $\mathrm{K}$ (Sigma) as recommended by manufacturer's instructions. Total RNAs were converted into cDNA using GoScript RTase (Promega) random hexamer primers for $60 \mathrm{~min}$ at $42^{\circ} \mathrm{C}$ according to manufacturer's instructions. cDNAs were then diluted 20 -fold and quantified by qPCR using SYBER green (Biorad) and specific primers. Data were acquired on CFX96 Real-Time PCR detection System (Bio-rad). Samples were analysed in triplicates and the expression level was calculated relative to zebrafish housekeeping gene EF1 $\alpha$. Sequence primers can be found in (supplementary data S3).

Immunostaining and in situ hybridization - Embryos were fixed overnight at $4^{\circ} \mathrm{C}$ in $\mathrm{BT}$-FIX, after which they were immediately processed or dehydrated and stored at $-20^{\circ} \mathrm{C}$ until use. After fixation or rehydration, embryos were washed twice with Phosphate Buffered Saline/1\% Triton X-100 (PBST), permeabilized with PBST/0.5\% Trypsin for $30 \mathrm{sec}$ and washed twice again with PBST. After blocking with PBST/10\% Fetal Calf Serum (FCS)/1\% bovine serum albumin (BSA) (hereafter termed 'blocking solution') for at least $1 \mathrm{~h}$, embryos were incubated with antibodies directed against either cleaved Caspase-3 (Asp175) (Cell Signaling Technology), or HuC/D 
(Molecular Probes), in blocking solution overnight at $4{ }^{\circ} \mathrm{C}$ followed by 5 washing steps with PBST. Embryos were then incubated with the appropriate Alexa Fluor-conjugated secondary antibodies (Molecular Probes) for at least $2 \mathrm{~h}$ at room temperature and washed three times. Fluorescent in situ hybridization was carried out as previously described (49). fli1a riboprobe preparation has been described elsewhere (50). Embryos were dissected, flat-mounted in glycerol and images were recorded on the confocal microscope TCS SP8 (Leica Microsystems) with an L $25 \times / 0.95$ W FLUOSTAR VIZIR objective (zoom X1.25) using the scanner resonant mode.

Comparison of Ribosome 3D structures - Atomic models of ribosomes from the following species: H. sapiens, PDB: 6EKO (Natchiar et al, 2017); P. falciparum, pdb: 6OKK and $3 J 79$ (51) ;T. gondii, PDB: 5XXU (40); T. vaginalis, PDB: 5XYI (40), L. donovani, PDB: 6AZ1, 6AZ3 (42); E. gracilis, PDB: 6ZJ3 (41) were displayed and aligned in UCSF Chimera (52) using the "Matchmaker" option, with the human 18S rRNA chain as reference. Prior to this structural alignment, cryo-EM maps of $P$. falciparum and T. gondii (EMD-2660 and EMD-6780, respectively) were visually inspected. Using UCSF Chimera and Coot (53), unattributed densities above C2065 in P. falciparum and C1764 in T. gondii cryo-EM map allowed to unambiguously replace unmodified residues by their $\operatorname{ac}^{4} \mathrm{C}$ counterparts in the corresponding atomic models.

Data availability statement - The data that support the findings of this study are available from the corresponding author [JC] upon reasonable request.

Disclosure declaration - The authors declare that they have no competing interests. 


\section{Acknowledgments}

We thank the many colleagues who kindly provided us with biological samples, particularly EMBRC-France (N. Turque, R. Lasbleiz), the Botanical garden of Toulouse, Insectosphere, Botanic-Blagnac, A. Mattout, S.Tournier, I. Massou, R. Jeanson, L. Distefano, P.Valenti, A. Dussutour, M. Betermier, J. Santos, K. Mochizuki, G. Allorent and P.M. Delaux. We are also grateful to Aurore Laire for excellent zebrafish care. This work was supported by Agence Nationale de la Recherche (ANR-18- CE12-0008-01). Research in the lab of JLM was supported by the Intramural Research Program of the NIH, National Cancer Institute, Center for Cancer Research (ZIA-BC011488). Research in the lab of D.L.J.L. is supported by the Belgian Fonds de la Recherche Scientifique (F.R.S./FNRS), the Université Libre de Bruxelles (ULB), the European Joint Programme on Rare Diseases (EJP-RD 'RiboEurope' and 'DBAGencure'), the Région Wallonne (SPW EER 'RIBOcancer'), the Internationale Brachet Stiftüng, and the Epitran COST action (CA16120). 


\section{References}

1. Bruenger, E., Kowalak, J.A., Kuchino, Y., McCloskey, J.A., Mizushima, H., Stetter, K.O. and Crain, P.F. (1993) 5S rRNA modification in the hyperthermophilic archaea Sulfolobus solfataricus and Pyrodictium occultum. FASEB journal : official publication of the Federation of American Societies for Experimental Biology, 7, 196-200.

2. Thomas, G., Gordon, J. and Rogg, H. (1978) N4-Acetylcytidine. A previously unidentified labile component of the small subunit of eukaryotic ribosomes. J Biol Chem, 253, 11011105.

3. Ito, S., Akamatsu, Y., Noma, A., Kimura, S., Miyauchi, K., Ikeuchi, Y. and Suzuki, T. (2014) A single acetylation of $18 \mathrm{~S}$ rRNA is essential for biogenesis of the small ribosomal subunit in Saccharomyces cerevisiae. J Biol Chem, 289, 26201-26212.

4. Ito, S., Horikawa, S., Suzuki, T., Kawauchi, H. and Tanaka, Y. (2014) Human NAT10 is an ATP-dependent RNA acetyltransferase responsible for N4-acetylcytidine formation in 18 S ribosomal RNA (rRNA). J Biol Chem, 289, 35724-35730.

5. Sharma, S., Langhendries, J.L., Watzinger, P., Kotter, P., Entian, K.D. and Lafontaine, D.L. (2015) Yeast Kre33 and human NAT10 are conserved 18S rRNA cytosine acetyltransferases that modify tRNAs assisted by the adaptor Tan1/THUMPD1. Nucleic acids research, 43, 2242-2258.

6. Sas-Chen, A., Thomas, J.M., Matzov, D., Taoka, M., Nance, K.D., Nir, R., Bryson, K.M., Shachar, R., Liman, G.L.S., Burkhart, B.W. et al. (2020) Dynamic RNA acetylation revealed by quantitative cross-evolutionary mapping. Nature, 583, 638-643.

7. Coureux, P.D., Lazennec-Schurdevin, C., Bourcier, S., Mechulam, Y. and Schmitt, E. (2020) Cryo-EM study of an archaeal 30S initiation complex gives insights into evolution of translation initiation. Commun Biol, 3, 58.

8. Johansson, M.J. and Bystrom, A.S. (2004) The Saccharomyces cerevisiae TAN1 gene is required for N4-acetylcytidine formation in tRNA. RNA, 10, 712-719.

9. Arango, D., Sturgill, D., Alhusaini, N., Dillman, A.A., Sweet, T.J., Hanson, G., Hosogane, M., Sinclair, W.R., Nanan, K.K., Mandler, M.D. et al. (2018) Acetylation of Cytidine in mRNA Promotes Translation Efficiency. Cell, 175, 1872-1886 e1824.

10. Tardu, M., Jones, J.D., Kennedy, R.T., Lin, Q. and Koutmou, K.S. (2019) Identification and Quantification of Modified Nucleosides in Saccharomyces cerevisiae mRNAs. ACS Chem Biol, 14, 1403-1409.

11. Liu, H.Y., Liu, Y.Y., Yang, F., Zhang, L., Zhang, F.L., Hu, X., Shao, Z.M. and Li, D.Q. (2020) Acetylation of MORC2 by NAT10 regulates cell-cycle checkpoint control and resistance to DNA-damaging chemotherapy and radiotherapy in breast cancer. Nucleic acids research, 48, 3638-3656.

12. Liu, X., Tan, Y., Zhang, C., Zhang, Y., Zhang, L., Ren, P., Deng, H., Luo, J., Ke, Y. and Du, X. (2016) NAT10 regulates p53 activation through acetylating p53 at K120 and ubiquitinating Mdm2. EMBO reports, 17, 349-366.

13. Shen, Q., Zheng, X., McNutt, M.A., Guang, L., Sun, Y., Wang, J., Gong, Y., Hou, L. and Zhang, B. (2009) NAT10, a nucleolar protein, localizes to the midbody and regulates cytokinesis and acetylation of microtubules. Experimental cell research, 315, 1653-1667. 
14. Balmus, G., Larrieu, D., Barros, A.C., Collins, C., Abrudan, M., Demir, M., Geisler, N.J., Lelliott, C.J., White, J.K., Karp, N.A. et al. (2018) Targeting of NAT10 enhances healthspan in a mouse model of human accelerated aging syndrome. Nature communications, $\mathbf{9}$, 1700.

15. Larrieu, D., Britton, S., Demir, M., Rodriguez, R. and Jackson, S.P. (2014) Chemical inhibition of NAT10 corrects defects of laminopathic cells. Science, 344, 527-532.

16. Sharma, S., Yang, J., van Nues, R., Watzinger, P., Kotter, P., Lafontaine, D.L.J., Granneman, S. and Entian, K.D. (2017) Specialized box C/D snoRNPs act as antisense guides to target RNA base acetylation. PLoS genetics, 13, e1006804.

17. Watkins, N.J. and Bohnsack, M.T. (2012) The box C/D and H/ACA snoRNPs: key players in the modification, processing and the dynamic folding of ribosomal RNA. Wiley interdisciplinary reviews. RNA, 3, 397-414.

18. Bachellerie, J.P., Michot, B., Nicoloso, M., Balakin, A., Ni, J. and Fournier, M.J. (1995) Antisense snoRNAs: a family of nucleolar RNAs with long complementarities to rRNA. Trends in biochemical sciences, 20, 261-264.

19. Kim, S.H., Spensley, M., Choi, S.K., Calixto, C.P., Pendle, A.F., Koroleva, O., Shaw, P.J. and Brown, J.W. (2010) Plant U13 orthologues and orphan snoRNAs identified by RNomics of RNA from Arabidopsis nucleoli. Nucleic acids research, 38, 3054-3067.

20. Tyc, K. and Steitz, J.A. (1989) U3, U8 and U13 comprise a new class of mammalian snRNPs localized in the cell nucleolus. EMBO J, 8, 3113-3119.

21. Essletzbichler, P., Konopka, T., Santoro, F., Chen, D., Gapp, B.V., Kralovics, R., Brummelkamp, T.R., Nijman, S.M. and Burckstummer, T. (2014) Megabase-scale deletion using CRISPR/Cas9 to generate a fully haploid human cell line. Genome Res, 24, 2059-2065.

22. Thomas, J.M., Briney, C.A., Nance, K.D., Lopez, J.E., Thorpe, A.L., Fox, S.D., BortolinCavaille, M.L., Sas-Chen, A., Arango, D., Oberdoerffer, S. et al. (2018) A Chemical Signature for Cytidine Acetylation in RNA. J Am Chem Soc, 140, 12667-12670.

23. Langhendries, J.L., Nicolas, E., Doumont, G., Goldman, S. and Lafontaine, D.L. (2016) The human box C/D snoRNAs U3 and U8 are required for pre-rRNA processing and tumorigenesis. Oncotarget, 7, 59519-59534.

24. Huang, Z.P., Zhou, H., He, H.L., Chen, C.L., Liang, D. and Qu, L.H. (2005) Genome-wide analyses of two families of snoRNA genes from Drosophila melanogaster, demonstrating the extensive utilization of introns for coding of snoRNAs. RNA, 11, 1303-1316.

25. Li, D., Wang, Y., Zhang, K., Jiao, Z., Zhu, X., Skogerboe, G., Guo, X., Chinnusamy, V., Bi, L., Huang, Y. et al. (2011) Experimental RNomics and genomic comparative analysis reveal a large group of species-specific small non-message RNAs in the silkworm Bombyx mori. Nucleic acids research, 39, 3792-3805.

26. Kiss, T. and Filipowicz, W. (1995) Exonucleolytic processing of small nucleolar RNAs from pre-mRNA introns. Genes \& development, 9, 1411-1424.

27. Smith, E.R., Lin, C., Garrett, A.S., Thornton, J., Mohaghegh, N., Hu, D., Jackson, J., Saraf, A., Swanson, S.K., Seidel, C. et al. (2011) The little elongation complex regulates small nuclear RNA transcription. Molecular cell, 44, 954-965.

28. Duveau, F. and Felix, M.A. (2012) Role of pleiotropy in the evolution of a cryptic developmental variation in Caenorhabditis elegans. PLoS biology, 10, e1001230. 
29. Dudnakova, T., Dunn-Davies, H., Peters, R. and Tollervey, D. (2018) Mapping targets for small nucleolar RNAs in yeast. Wellcome open research, 3, 120.

30. Dewe, J.M., Whipple, J.M., Chernyakov, I., Jaramillo, L.N. and Phizicky, E.M. (2012) The yeast rapid tRNA decay pathway competes with elongation factor $1 \mathrm{~A}$ for substrate tRNAs and acts on tRNAs lacking one or more of several modifications. RNA, 18, 18861896.

31. Ding, F., Li, H.H., Zhang, S., Solomon, N.M., Camper, S.A., Cohen, P. and Francke, U. (2008) SnoRNA Snord116 (Pwcr1/MBII-85) deletion causes growth deficiency and hyperphagia in mice. PloS one, 3, e1709.

32. Hebras, J., Marty, V., Personnaz, J., Mercier, P., Krogh, N., Nielsen, H., Aguirrebengoa, M., Seitz, H., Pradere, J.P., Guiard, B.P. et al. (2020) Reassessment of the involvement of Snord115 in the serotonin $2 c$ receptor pathway in a genetically relevant mouse model. elife, 9.

33. Lee, J., Harris, A.N., Holley, C.L., Mahadevan, J., Pyles, K.D., Lavagnino, Z., Scherrer, D.E., Fujiwara, H., Sidhu, R., Zhang, J. et al. (2016) Rpl13a small nucleolar RNAs regulate systemic glucose metabolism. The Journal of clinical investigation, 126, 4616-4625.

34. Skryabin, B.V., Gubar, L.V., Seeger, B., Pfeiffer, J., Handel, S., Robeck, T., Karpova, E., Rozhdestvensky, T.S. and Brosius, J. (2007) Deletion of the MBII-85 snoRNA gene cluster in mice results in postnatal growth retardation. PLoS genetics, 3, e235.

35. Soeno, Y., Fujita, K., Kudo, T., Asagiri, M., Kakuta, S., Taya, Y., Shimazu, Y., Sato, K., Tanaka-Fujita, R., Kubo, S. et al. (2013) Generation of a mouse model with downregulated U50 snoRNA (SNORD50) expression and its organ-specific phenotypic modulation. PloS one, 8, e72105.

36. Ben-Shem, A., Garreau de Loubresse, N., Melnikov, S., Jenner, L., Yusupova, G. and Yusupov, M. (2011) The structure of the eukaryotic ribosome at 3.0 A resolution. Science, 334, 1524-1529.

37. Natchiar, S.K., Myasnikov, A.G., Kratzat, H., Hazemann, I. and Klaholz, B.P. (2017) Visualization of chemical modifications in the human $80 \mathrm{~S}$ ribosome structure. Nature, 551, 472-477.

38. Sharma, S. and Lafontaine, D.L.J. (2015) 'View From A Bridge': A New Perspective on Eukaryotic rRNA Base Modification. Trends in biochemical sciences, 40, 560-575.

39. Tamm, T., Kisly, I. and Remme, J. (2019) Functional Interactions of Ribosomal Intersubunit Bridges in Saccharomyces cerevisiae. Genetics, 213, 1329-1339.

40. Li, Z., Guo, Q., Zheng, L., Ji, Y., Xie, Y.T., Lai, D.H., Lun, Z.R., Suo, X. and Gao, N. (2017) Cryo-EM structures of the $80 \mathrm{~S}$ ribosomes from human parasites Trichomonas vaginalis and Toxoplasma gondii. Cell research, 27, 1275-1288.

41. Matzov, D., Taoka, M., Nobe, Y., Yamauchi, Y., Halfon, Y., Asis, N., Zimermann, E., Rozenberg, H., Bashan, A., Bhushan, S. et al. (2020) Cryo-EM structure of the highly atypical cytoplasmic ribosome of Euglena gracilis. Nucleic acids research, 48, 1175011761.

42. Shalev-Benami, M., Zhang, Y., Rozenberg, H., Nobe, Y., Taoka, M., Matzov, D., Zimmerman, E., Bashan, A., Isobe, T., Jaffe, C.L. et al. (2017) Atomic resolution snapshot of Leishmania ribosome inhibition by the aminoglycoside paromomycin. Nature communications, 8, 1589. 
43. Cavaille, J. and Bachellerie, J.P. (1998) SnoRNA-guided ribose methylation of rRNA: structural features of the guide RNA duplex influencing the extent of the reaction. Nucleic acids research, 26, 1576-1587.

44. Montellese, C., Montel-Lehry, N., Henras, A.K., Kutay, U., Gleizes, P.E. and O'Donohue, M.F. (2017) Poly(A)-specific ribonuclease is a nuclear ribosome biogenesis factor involved in human $18 \mathrm{~S}$ rRNA maturation. Nucleic acids research, 45, 6822-6836.

45. Martineau, Y., Azar, R., Muller, D., Lasfargues, C., El Khawand, S., Anesia, R., Pelletier, J., Bousquet, C. and Pyronnet, S. (2014) Pancreatic tumours escape from translational control through 4E-BP1 loss. Oncogene, 33, 1367-1374.

46. Yang, J., Sharma, S., Watzinger, P., Hartmann, J.D., Kotter, P. and Entian, K.D. (2016) Mapping of Complete Set of Ribose and Base Modifications of Yeast rRNA by RP-HPLC and Mung Bean Nuclease Assay. PloS one, 11, e0168873.

47. Alestrom, P., D'Angelo, L., Midtlyng, P.J., Schorderet, D.F., Schulte-Merker, S., Sohm, F. and Warner, S. (2020) Zebrafish: Housing and husbandry recommendations. Laboratory animals, 54, 213-224.

48. Kimmel, C.B., Ballard, W.W., Kimmel, S.R., Ullmann, B. and Schilling, T.F. (1995) Stages of embryonic development of the zebrafish. Developmental dynamics : an official publication of the American Association of Anatomists, 203, 253-310.

49. Quillien, A., Moore, J.C., Shin, M., Siekmann, A.F., Smith, T., Pan, L., Moens, C.B., Parsons, M.J. and Lawson, N.D. (2014) Distinct Notch signaling outputs pattern the developing arterial system. Development, 141, 1544-1552.

50. Lawson, N.D., Scheer, N., Pham, V.N., Kim, C.H., Chitnis, A.B., Campos-Ortega, J.A. and Weinstein, B.M. (2001) Notch signaling is required for arterial-venous differentiation during embryonic vascular development. Development, 128, 3675-3683.

51. Wong, W., Bai, X.C., Brown, A., Fernandez, I.S., Hanssen, E., Condron, M., Tan, Y.H., Baum, J. and Scheres, S.H. (2014) Cryo-EM structure of the Plasmodium falciparum $80 \mathrm{~S}$ ribosome bound to the anti-protozoan drug emetine. eLife, 3.

52. Pettersen, E.F., Goddard, T.D., Huang, C.C., Couch, G.S., Greenblatt, D.M., Meng, E.C. and Ferrin, T.E. (2004) UCSF Chimera--a visualization system for exploratory research and analysis. Journal of computational chemistry, 25, 1605-1612.

53. Emsley, P., Lohkamp, B., Scott, W.G. and Cowtan, K. (2010) Features and development of Coot. Acta crystallographica. Section D, Biological crystallography, 66, 486-501. 


\section{Figure - Legends}

Figure 1 - Schematic of SNORD13-dependent ribosomal RNA acetylation. A) Through an incompletely defined antisense-mediated mechanism, human SNORD13 assists the RNA cytidine acetyltransferase NAT10 in modifying C1842 in the universally conserved helix 45 at the 3' extremity of $18 \mathrm{~S}$ rRNA. B) Helix 45 is enriched with post-transcriptional RNA modifications. RNA modifying enzymes and their modified nucleotides (lollipops) are indicated: SSU-ac ${ }^{4} \mathrm{C} 1842$ (yellow), SSU-m ${ }^{6} \mathrm{~A} 1832$ (green) and $\mathrm{SSU}-\mathrm{m}_{2}{ }^{6} \mathrm{~A} 1850$ and $\mathrm{SSU}-\mathrm{m}_{2}{ }^{6} \mathrm{~A} 1851$ (orange). C) Human SNORD13 base-pairs with 18S rRNA sequences on each side of the cytidine to be acetylated. Note that SNORD13-rRNA duplexes tolerate G-U wobble base-pairs, mismatches or even bulged nucleotides and the length of the intervening loops, from either rRNA or SNORD side, also differ from one organism to another (Supplementary figure S1).

Figure 2 - The specific lack of SNORD13-mediated acetylation in helix 45 does not alter cell growth or translation. A) Schematic representation of SNORD13 locus at human chromosome 8 . The relative location of the two DNA sequences targeted by sgRNAs is shown (PAM sequence is underlined). B) Northern blot showing that SNORD13 is no longer detected in clones bearing deletions. * indicate truncated SNORD13 form which is routinely detected and corresponding to RNA species whose 5'-end is positioned 5-6 nucleotides upstream of the Cbox (not shown). C) RP-HPLC chromatograms of nucleosides obtained from helix 45 of WT (black) and SNORD13 (red) cells. Peak corresponding to SSU-ac ${ }^{4}$ C1842 is indicated.

Sanger DNA sequencing of RT-PCR products obtained after borohydride treatments of total RNA extracted from WT and SNORD13-KO cells. \% of misincorporation (C-to-U) at SSUC1842 (helix 45) and SSU-C1337 (helix 34) is indicated below each electropherogram. E) Growth curves of WT and SNORD13-KO cells as judged by cell counting ( $\mathrm{n}=6$ for each genotype). F) Apoptosis levels determined by flow cytometry using propidium and Annexin $\mathrm{V}$ 
staining of WT and SNORD13-KO cells ( $\mathrm{n}=2$ for each genotype). G) SUnSET assay. Incorporation of puromycin into the elongating peptide was assayed in WT and SNORD13-KO cells (western blotting with anti-puromycin antibodies). Ponceau S staining of the membrane and detection of CDK9 (western blotting) were used as gel loading controls. Cells treated with the translation inhibitor cycloheximide $(\mathrm{CHX})$ were also used as negative controls. $\mathbf{H})$ Luciferase reporter gene assays. WT and SNORD13-KO cells were transiently co-transfected by two plasmids expressing Renilla luciferase gene (LucR; internal control) or Firefly luciferase gene (lucF; translation reporter) carrying either an in-frame stop-codons (UAA, UAG), a detrimental mutation (R-to-S) or an IRES from FGF1A, FGF2 and CMV. Histograms show the normalized luciferase activity (lucF-to-lucR ratio). Data are expressed as mean +/- s.e.m. and represent 5-6 independent experiments with triplicate measurements.

Figure 3 - Phenotypic analyses of SNORD13-deficient zebrafish embryos. A) Schematic representation of the zebrafish snord13 locus. The relative location of the two DNA sequences targeted by sgRNAs is shown (PAM sequence is underlined). B) Northern blotting showing that SNORD13 is no longer detected in embryos bearing deletion events. *: truncated SNORD13 form which is routinely detected. SNORD118 was used as a gel loading control. C) Histograms show percentage of misincorporation ( $\mathrm{C}-\mathrm{to}-\mathrm{U}$ ratio) measured in borohydride-treated RNA samples prepared from WT and SNORD13-KO embryos $(n=3)$. D-G Confocal projections of WT (D, F) and SNORD13-KO (E, G) embryos after immunostaining against cleaved Caspase-3 (red) and the pan neural marker HuC/D (green). D-E: Dorsal view of the zebrafish brain at 30 hpf, neurons from the olfactory epithelium (oe), tectum (t), pineal gland (pg), optic tectum (ot) and trigeminal ganglion (tg) can be detected. F-G: Lateral view of the spinal cord (sc) at $30 \mathrm{hpf}$. H-I Confocal projections of WT (H) and SNORD13-KO (I) embryos after in situ RNA hybridization using antisense riboprobes directed against fli1a transcripts. White brackets indicate the dorsal aorta (DA) and the cardinal vein (CV) and the white arrows point to 
intersegmental vessels. Pictures are representative of three independent experiments (at least 6 embryos imaged per genotype).

Figure 4 - Cross-evolutionary survey of eukaryotic rRNA acetylation machinery. A) This table summarizes the conservation of the 5'-CCG-3' motif, the acetylation status of helix 45 and the detection of SNORD13 and NAT10 genes in commonly used models or organisms for which we experimentally assayed the presence of $\operatorname{ac}^{4} \mathrm{C}$ at helix 45. n.d.: not determined. n/a: not applicable (genome is not available). B) Simplified phylogenetic tree of Metazoan. The genomic organization (intronic vs intergenic) of newly-identified SNORD13 genes (red arrows) is shown. Note that intronic SNORD13 can be positioned in the sense or antisense orientation with respect to their host-genes (black arrow). C) Simplified phylogenetic tree of Archaeplastida. Acetylation status of helix 45 and the presence of SNORD13 in the genome of representative species are indicated. Note that while rRNA in algal species is acetylated, we failed to identify obvious SNORD13 counterparts. A full list of newly-identified SNORD13 sequences can be found in Supplementary data S1.

\section{Figure 5 - Atypical SNORD13-related RNA guides rRNA acetylation in D. melanogaster. A)}

Simplified phylogenetic tree of Arthropoda. Representative species where SNORD13-like were identified are indicated. B) Simplified phylogenetic tree of Diptera. The genomic arrangements of newly-identified SNORD13 genes (blue arrow) are shown. C) Multiple sequence alignment of representative eukaryotic SNORD13 sequences. The small red arrow indicates the relative position of the mature 5'-end of Or-CD1 in Diptera (see also panel F) while the two horizontal black arrows in opposite orientation depict the terminal 5'-3' stem structure. Note that SNORD13 sequences in Diptera - but not in other species - lack one of the two conserved antisense rRNA elements (denoted by horizontal blue bars). Vertical black, grey and white bars highlight Diptera, other Arthropoda and non-Arthropoda species, respectively. D) Immunoprecipitation by anti- 
trimethyl cap (R1131) antibodies. OR-CD1 in various insects (as indicated above the panels) was detected by Northern blots. The same membrane was hybridized with an antisense oligo probes that recognized uncapped 5.8S rRNA used as negative controls. Input RNA (I); RNA recovered from the pellet $(P)$, RNA recovered from the supernatant $(S)$. The theoretical size of SNORD13 (nt) in each of the species studied is indicated in parentheses. E) Schematic representation of two distinct modes of expression: independent transcription gives rise to short (top) or long (bottom) forms of 5' capped SNORD13. F) A mutant KO fly strain harboring an inserted P-element in the Or-CD1 gene (DmellP\{EP\}snoRNA:Or-CD1G9117) does not express OR-CD1 as assayed by primer extension. Note that the 5'-end of the cDNA product confirms that fly Or-CD1 lacks one 18S rRNA complementarity. The expected size (nt) of OR-CD1 with 4-5 nucleotides upstream of the C-box (as shown on the panel) is indicated in parentheses. ${ }^{32} \mathrm{P}$ labeled primer (P). G) Misincorporation (C-to-U) at SSU-C1968 (helix 45) as judged by Sanger DNA sequencing of RT-PCR products obtained after borohydride treatments of total RNA extracted from WT and Or-CD1-KO adult flies.

Figure 6 - Life without rRNA acetylation A) Simplified phylogenetic tree of Nematoda. Sequence contexts of the acetylated cytidine in representative species of each clade are indicated. B) Schematic representations of helix 45. Green bases represent compensatory base changes, as compared to human helix 45 , while red bases provoke irregularity in the geometry of helix 45 . The triplet sequence motif is framed. C) Sanger DNA sequencing of RTPCR products obtained after borohydride treatments of total RNA extracted from C. elegans. D) $A C^{4} C$ content in total RNA extracted from adult $C$. elegans was visualized by Northern blottingbased assay using anti-Ac ${ }^{4} \mathrm{C}$ antibodies. Ethidium bromide (etBr) staining indicates the relative position of rRNA and tRNA species. E) Top-panels: WT and $\Delta s n R 4 \Delta s n R 45$ yeast strains were grown at $30^{\circ} \mathrm{C}$ (left) or $37^{\circ} \mathrm{C}$ (right) in liquid YPD. Optical density at $600 \mathrm{~nm}\left(\mathrm{OD}_{600}\right)$ was measured every 30 minutes. Growth curves of individual biological samples (thin lines; $n=3$ or 
12 for $30^{\circ} \mathrm{C}$ and $37^{\circ} \mathrm{C}$ samples, respectively) and the mean of replicates (thick lines) are presented along with a confidence interval (gray area). Bottom-panels: WT and $\Delta \mathrm{snR} 4 \Delta \mathrm{snR} 45$ yeast strains were spotted on a YPD-agar plate and incubated for 48 hours at $30^{\circ} \mathrm{C}$ or $37^{\circ} \mathrm{C}$. Cells were grown in duplicates with a 5-fold dilution between distinct concentrations.

Figure 7 - Comparative schematic representation of rRNA modification systems. A) Through the formation of a perfect (or near perfect) RNA duplex ( 10-20 bp), SNORDs guide Fibrillarin/Nop1 to their target rRNA nucleotides, i.e. the nucleotide to be 2'-O-methylated (2'-O$\mathrm{Me}$ ) is paired to the fifth position upstream of the conserved D (or D') box. B) Through the formation of two perfect (or near perfect) RNA duplexes ( 4-10 bp each), SNORAs guide Dyskerin/Cbf5 to their target rRNA nucleotides, i.e. the uridine to be isomerized into pseudouridine $(\Psi)$ remains unpaired and it is located at $\sim 14-16 \mathrm{nt}$ from the conserved $\mathrm{H}$ (or ACA) box. C) snR45, snR4 and SNORD13 contain two imperfect antisense elements matching either side of the cytidine to be acetylated $\left(\operatorname{ac}^{4} \mathrm{C}\right)$ and, through still unknown mechanisms, guide cytosine acetylation by NAT10/Kre33. D) In D. melanogaster, SNORD13 targets rRNA acetylation at helix 45 through the use of a single antisense rRNA sequence.

\section{Supplementary figure S1 - Examples of SNORD13-rRNA duplexes across evolution.} Schematic representations of base-pairing interactions between SNORD13 and 18S rRNA (only a few representative examples are shown). The targeted $\mathrm{C}$ for acetylation is written in green.

\section{Supplementary figure S2 - Loss of SNORD13 does not affect pre-rRNA synthesis and} processing. A-B) Northern blot - Total RNA prepared from WT and SNORD13-KO clones, as well as from a pool of the three WT and KO clones, were analysed by Northern blot using probe 1 (5-ITS1) and probe 2 (ITS2) that detect main rRNA precursors intermediates as depicted in panel B). Cytoplasmic $18 \mathrm{~S}$ and $28 \mathrm{~S}$ rRNAs were also visualized by ethidium bromide staining of 
a denaturing $1.2 \%$ agarose gel. Relative quantification of rRNA intermediates based on three independent Northern blots did not reveal any obvious abnormalities (not shown). C) RNAse H assay - Total RNA extracted from WT or SNORD13-KO cells were annealed with antisense DNA oligonucleotide (black line), RNAse $\mathrm{H}$ digestion products were then resolved onto a $12 \%$ acrylamide gels and visualized by Northern blot with an antisense oligo-probe matching the 3'end of $18 \mathrm{~S}$ (red line). The ladder-like pattern reflecting heterogeneity of the 3 '-end at $18 \mathrm{~S}$ in SNORD13-KO cells was indistinguishable from that of observed in WT cells, indicating that the accuracy of cleavages that delineates the 3 ' extremity of 185 rRNA is not impaired in SNORD13-KO cells. D) Pulse chase experiments - WT and SNORD13-KO cells were pulselabelled with $\mathrm{L}-\left[\right.$ methyl- $\left.{ }^{3} \mathrm{H}\right]-$ methionine and harvested by chase times as indicated above the panel. Freshly-synthesized rRNA intermediates were detected by autoradiography after being separated by electrophoresis on denaturing $1.2 \% \mathrm{gel}$ and transferred to a nylon membrane. Dynamics of rRNA synthesis in the SNORD13-KO cells was in the normal range, as shown by the timing of appearance and lifespan of the detected methyl- ${ }^{3} \mathrm{H}$ labelled rRNA precursors. E) Polysome profiling - Whole cytoplasmic extracts prepared from WT and (left) SNORD13-KO (right) cells were fractioned by ultracentrifugation on a $10-50 \%$ sucrose gradient. Absorbance $(254 \mathrm{~nm})$ of each fraction was recorded and the peaks corresponding to free $40 \mathrm{~S}$ and $60 \mathrm{~S}$ subunits, $80 \mathrm{~S}$ ribosomes (monosomes) and polysomes are indicated.

\section{Supplementary figure S3 - Deletion of snord13 gene does not perturb zebrafish} development. A) Bright field images of WT (up) and homozygous (down) snord13 mutant embryos derived from the crossing of homozygous snord13 females with heterozygous males. Pictures are representative of three independent crosses. B) Relative mRNA expressions determined by RT-qPCR in 30 hpf WT and SNORD13-KO embryos (3 independent experiments with at least 15 animals per condition). The tested genes are listed below the histogram (black and red histograms correspond to WT and KO, respectively). 
Supplementary figure S4 - Phylogenetic conservation of helix 45 across evolution. Sequence (A) and schematic representations (B) of helix 45 in representative species belonging to each eukaryotic supergroup. Green bases represent compensatory base changes, as compared to human helix 45 , while red bases alter the geometry of helix 45 . The triplet sequence motif is framed.

Supplementary figure S5 - Probing rRNA acetylation in T. thermophila, S. carpocapsae and $\boldsymbol{P}$. polycephalum. Sanger DNA sequencing of RT-PCR products obtained after borohydride treatments of total RNA extracted from $P$. polycephalum (left) $T$. thermophila (middle), S. carpocapsae (right). \% of misincorporation at helix 45 is indicated below each electropherogram.

Supplementary figure S6 - rRNA h45 acetylation does not seem to affect Rpl41 positioning. Spatial alignment of Ribosomal 3D structures of various species, carrying either acetylated C (left panel) or unmodified $U$ or G (right panel) on rRNA helix 45 (h45) revealed no change in the location of ribosomal protein Rpl41 (eL41). For each aligned species (indicated on the top left of each vignette), the modified/unmodified nucleotide of interest is shown both on the structure as well as on the 3-nucleotide sequence by an underlined character. For spatial cueing, lateral chain of an N-terminal Arginine of Rpl41, one of the closest aminoacids of the nucleotide of interest, is fully displayed.

\section{Supplementary data S1 - Examples of newly-identified SNORD13 sequences.}

\section{Supplementary data S2 - The primary structure of NAT10 across evolution}

\section{Supplementary data S3 - Sequences of primers}


bioRxiv preprint doi: https://doi.org/10.1101/2021.11.30.470322; this version posted November 30, 2021. The copyright holder for this preprint (which was not certified by peer review) is the author/funder. All rights reserved. No reuse allowed without permission. 
bioRxiv preprint doi: https://doi.org/10.1101/2021.11.30.470322; this version posted November 30, 2021. The copyright holder for this preprint (which was not certified by peer review) is the author/funder. All rights reserved. No reuse allowed without permission.

A
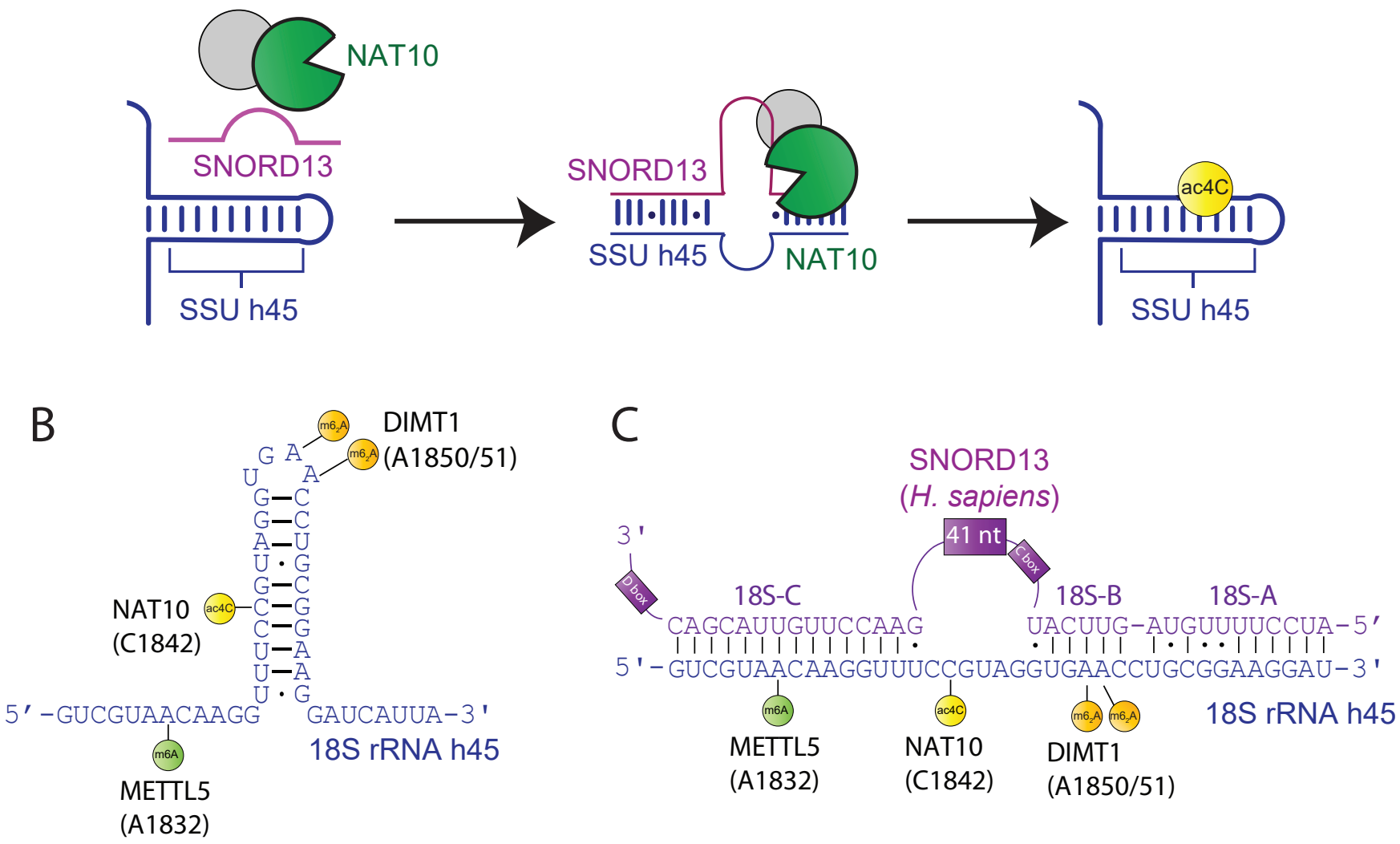
bioRxiv preprint doi: https://doi.org/10.1101/2021.11.30.470322; this version posted November 30, 2021. The copyright holder for this preprint (which was not certified by peer review) is the author/funder. All rights reserved. No reuse allowed without permission.
A
B

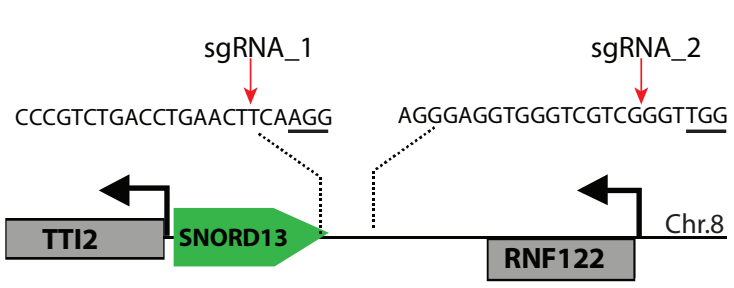

D

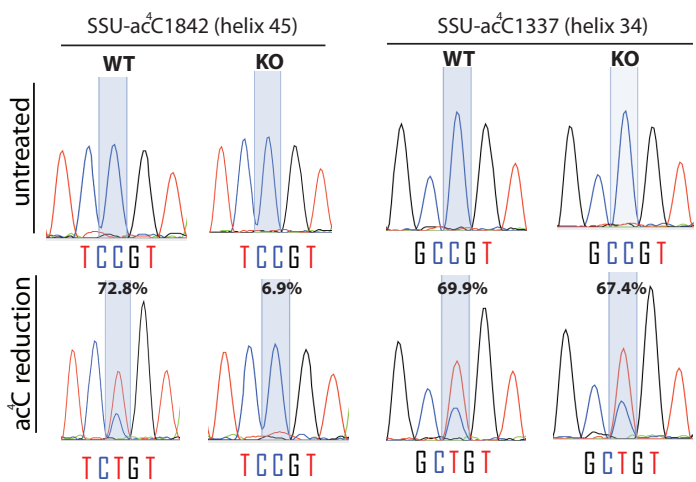

G

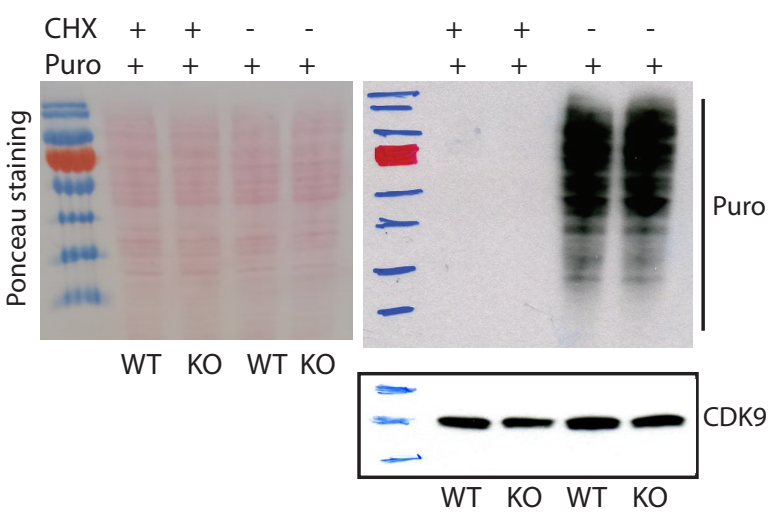

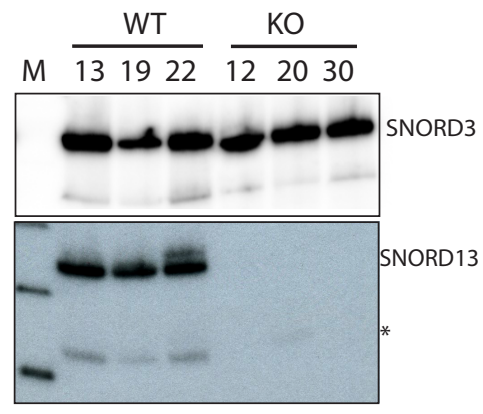

$E$
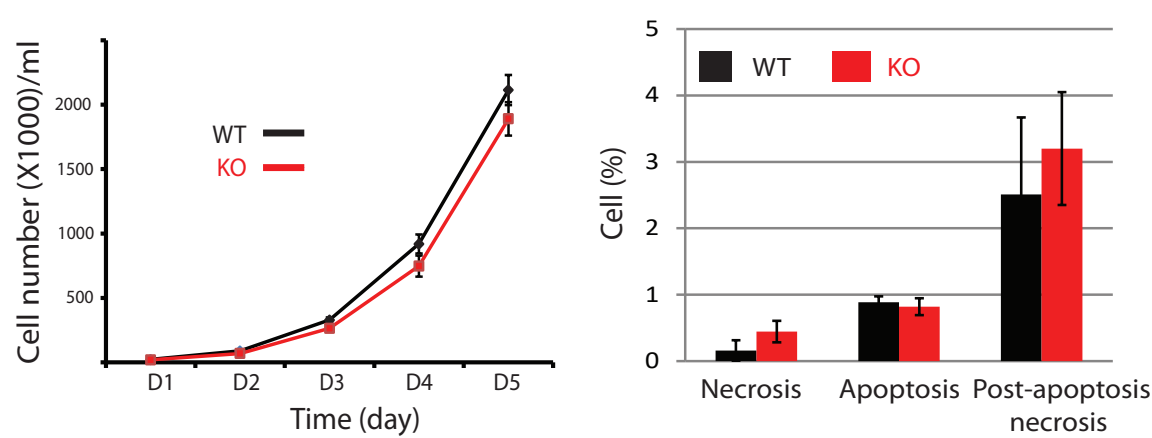

$\mathrm{H}$

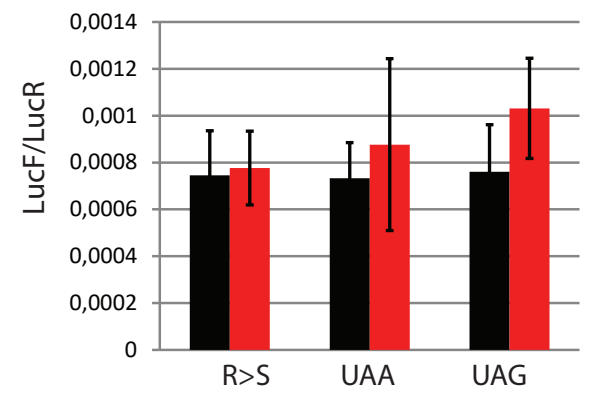

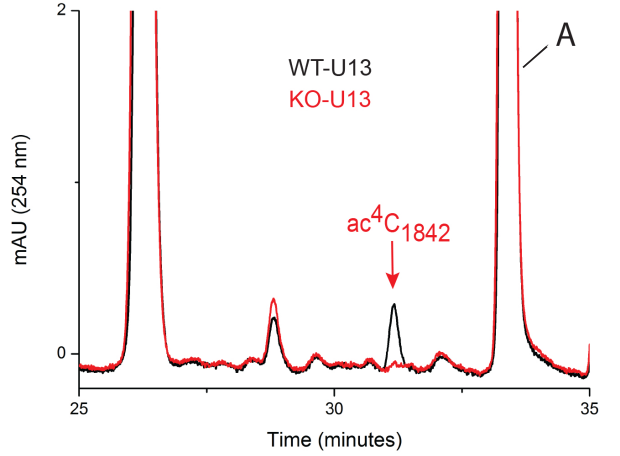

F
WT

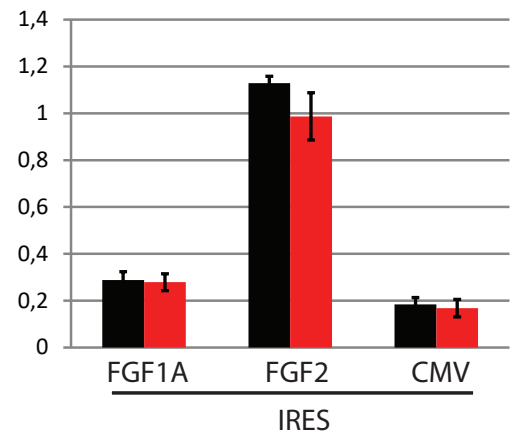


bioRxiv preprint doi: https://doi.org/10.1101/2021.11.30.470322; this version posted November 30, 2021. The copyright holder for this preprint (which was not certified by peer review) is the author/funder. All rights reserved. No reuse allowed without permission.

A

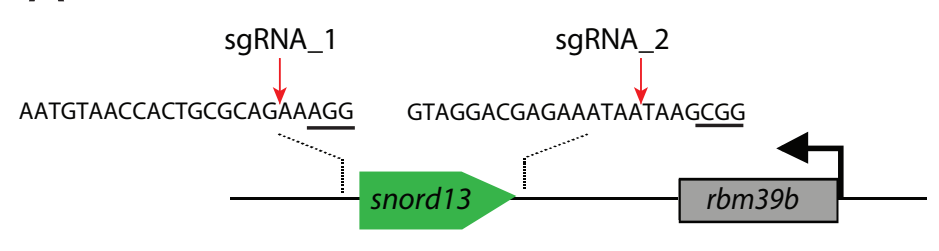

B

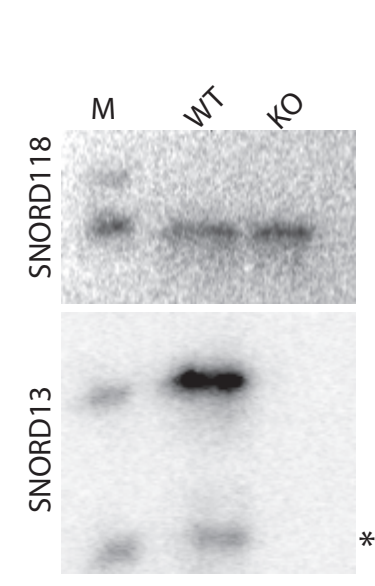

C

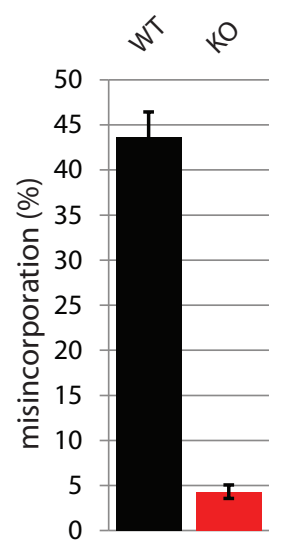

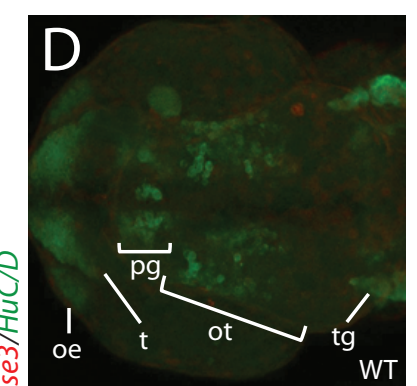
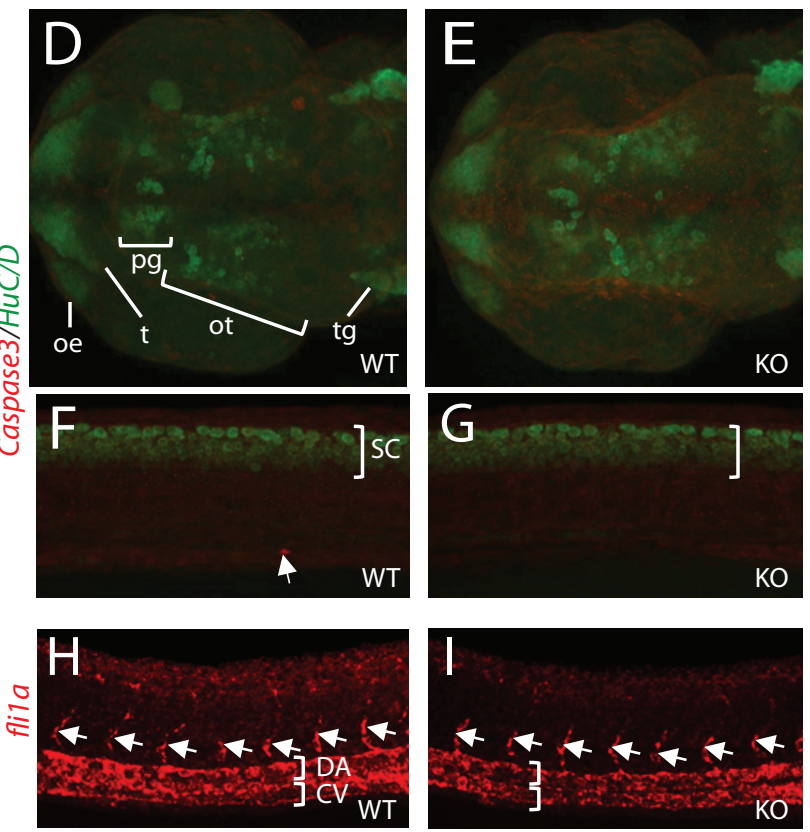

Bortolin-Cavaillé et al - Figure 3 
bioRxiv preprint doi: https://doi.org/10.1101/2021.11.30.470322; this version posted November 30, 2021. The copyright holder for this preprint (which was not certified by peer review) is the author/funder. All rights reserved. No reuse allowed without permission.

A

\begin{tabular}{|c|c|c|c|c|c|}
\hline & Organisms & Helix 45 & Ac4C & SNORD & NAT10 \\
\hline & H. sapiens & CCG & yes (Sharma et al 2015) & SNORD13 (U13) & yes \\
\hline & M. musculus (mouse) & CCG & n.d. & SNORD 13-like (this study) & yes \\
\hline & R. norvegicus (rat) & CCG & yes (Thomas et al 1978) & SNORD13-like (this study) & yes \\
\hline & G. gallus (chicken) & CCG & yes (Thomas et al 1978) & GGN86 (Makarova et al 2011) & $\begin{array}{l}\text { yes } \\
\text { yes }\end{array}$ \\
\hline & X. laevis (frog) & CCG & n.d. & SNORD13-like (this study) & yes \\
\hline & D. rerio (zebrafish) & CCG & yes (this study) & SNORD13-like (this study) & yes \\
\hline & P. marinus (lamprey) & CCG & n.d. & SNORD13-like (this study) & yes \\
\hline & C. intestinalis (sea squirt) & CCG & n.d. & SNORD13-like (this study) & yes \\
\hline & B. floridae (Florida lancelet) & CCG & n.d. & SNORD13-like (this study) & yes \\
\hline & S. purpuratus (purple sea urchin) & CCG & n.d. & SNORD13-like (this study) & yes \\
\hline & H. attenuata (star fish) & CCG & yes (this study) & $n / a$ & $n / a$ \\
\hline & A. mellifera (bee) & CCG & yes (this study) & SNORD13-like (this study) & yes \\
\hline & A. gambiae (mosquito) & C৫G & n.d. & not found & $\begin{array}{l}\text { yes } \\
\text { yes }\end{array}$ \\
\hline & D. melanogaster (fruit fly) & CCG & yes (this study) & OR-CD1 (this study) & $\begin{array}{l}\text { yes } \\
\text { yes }\end{array}$ \\
\hline & B. mori (domestic silk moth) & CCG & & Bm_10 (this study) & $\begin{array}{l}\begin{array}{l}\text { es } \\
\text { yes }\end{array} \\
\text { a }-10\end{array}$ \\
\hline Metazoan I & A. labyrinthica (spider) & CCG & yes (this study) & $\mathrm{n} / \mathrm{a}^{-}$ & n/a \\
\hline Metazoan & C. scutellaris (ant) & CCG & yes (this study) & $\mathrm{n} / \mathrm{a}$ & $\begin{array}{l}n / d \\
n / a\end{array}$ \\
\hline & I. scapularis (deer tick) & CCG & n.d & SNORD13-like (this study) & $\begin{array}{l}n / a \\
\text { yes }\end{array}$ \\
\hline & C. elegans (nematode) & CUG & no (this study) & not found & $\begin{array}{l}\text { yes } \\
\text { yes }\end{array}$ \\
\hline & S. carpocapsae (nematode) & CCG & yes (this study) & not found & $\begin{array}{l}\text { yes } \\
\text { yes }\end{array}$ \\
\hline & H. aspera (snail, mollusk) & CCG & yes (this study) & $\mathrm{n} / \mathrm{a}$ & yes \\
\hline & M. galloprovincialis (mussel, mollusk) & CCG & yes (this study) & SNORD13-like (this study) & n/a \\
\hline & A. californica (aplysia mollusk) & CCG & n.d. & SNORD 13-like (this study) & $\begin{array}{l}\text { yes } \\
\text { yes }\end{array}$ \\
\hline & 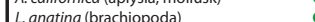 & CCG & nd & SNORD13-like (this study) & yes \\
\hline & O. bimaculoides (octopus) & CCG & n.d. & $\begin{array}{l}\text { SNORD 13-like (this this study) } \\
\text { SNOdy) }\end{array}$ & yes \\
\hline & A. viridis (anemona cnidaria) & CCG & yes (this study) & n/a & yes \\
\hline & 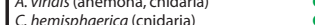 & CCG & $\begin{array}{l}\text { yes (this study) } \\
\text { yes (this study) }\end{array}$ & & $\mathrm{n} / \mathrm{a}$ \\
\hline & $\begin{array}{l}\text { C. hemisphaerica (cnidarla) } \\
\text { P. aracillis (cnidaria) }\end{array}$ & CCG & $\begin{array}{l}\text { yes (this study) } \\
\text { yes (this study) }\end{array}$ & $\begin{array}{l}\text { SNORD 13-like (this study) } \\
\text { na }\end{array}$ & yes \\
\hline & & CCG & yes (this study) & $\begin{array}{l}\text { n/a } \\
\text { SNORD13-like (this study) }\end{array}$ & $\mathrm{n} / \mathrm{a}$ \\
\hline & N. vectensis (Starlet sea anemone, cnidaria) & SCG & n.a. & SNORD 13-like (this study) & yes \\
\hline & D. gigantea (coral, cnidaria) & CCG & n.d. & & yes \\
\hline & D. australiensis (sponge) & CCG & yes (this study) & $\mathrm{n} / \mathrm{a}$ & $\mathrm{n} / \mathrm{a}$ \\
\hline & Haliclona sp (sponge) & CCG & yes (this study) & $\mathrm{n} / \mathrm{a}$ & $\mathrm{n} / \mathrm{a}$ \\
\hline & IE. muelleri (sponge) & CCG & n.d. & SNORD13-like (this study) & yes \\
\hline & U. maydis (corn smut fungus) & CUG & no & not found & yes \\
\hline & m Paris») & $\mathrm{cc}$ & yes (this study) & not found & yes \\
\hline & S. pombe (fission yeast) & CCG & yes (Taoka et al 2014) & not found & yes \\
\hline Fungi & N. crassa (filamentous fungu) & CCG & n.d. & not found & yes \\
\hline & P. anserina (filamentous fungu) & CCG & n.d. & not found & yes \\
\hline & A. niger (filamentous fungu) & CCG & n.d. & not found & yes \\
\hline & S. cerevisiae (budding yeast) & CCG & yes (Ito et al 2014) & SnR45 (Sharma et al 2017) & yes \\
\hline Amoebozoa & D. discoideum (social amoeba & CCG & yes (McCarrol et & not found & yes \\
\hline & P. polycephalum (slime mod) & UCG & yes (this study) & not found & yes \\
\hline & A. thal & CCG & yes $(s h$ & $/ 108 / 2 / 2$ & 2010) yes \\
\hline & & CCG & n.d. & SNORD13-like (this study) & yes \\
\hline & A. trichopoda (ancestor of angiosperms) & CCG & n.d & SNORD13-like (this study) & yes \\
\hline & N. colorata (water lily) & CCG & n.d. & SNORD13-like (this study) & yes \\
\hline & C. revoluta (Japanese sago palm) & CCG & yes (this study) & & $n / a$ \\
\hline & S. moellendorffii (lycophyte) & CCG & n.d & SNORD13-like (this study) & yes \\
\hline Archaeplastida & P. patens (moss) & CCG & n.d & SNORD13-like (this study) & yes \\
\hline Arctiatepistiud & H. cupressiforme (moss) & CCG & yes (this study) & & $\mathrm{n} / \mathrm{a}$ \\
\hline & M. polymorpha (liverwort) & CCG & n.d. & SNORD13-like (this study) & yes \\
\hline & C. braunii (freshwater algae) & CCG & n.d. & SNORD13-like (this study) & yes \\
\hline & C. reinhardtii (green algae) & CCG & yes (this study) & not found & yes \\
\hline & G. sulphuraria (red algae) & CCG & yes (this study) & not found & yes \\
\hline & C. crispus (red algae) & CCG & n.d. & not found & yes \\
\hline & Ectoc & CCG & & & yes \\
\hline & liatom, microalgae) & CCG & his study) & not found & yes \\
\hline TSAR & T. thermophila (ciliata) & CUG & no (this study) & not found & yes \\
\hline ISAK & P. tetraurelia (ciliata) & CCG & yes (this study) & not found & yes \\
\hline & P. falciparum (agent of malaria, parasite) & CCG & yes (this study) & not found & yes \\
\hline & T. gondii (agent of toxoplasmosis, parasite) & CCG & yes (N. Gao, pers. com.) & not found & yes \\
\hline & tinalis (flagellated protozan parasite) & e)CCG & n.d. & not found & \\
\hline & (flagellated protozan parasite) & CGG & no & not found & yes \\
\hline vat & lagellated green algae) & Cuc & no & not found & to \\
\hline & & $\mathrm{Cu}$ & no & not found & yes \\
\hline & major (flagellated protozan parasite & CUG & no & not found & es \\
\hline
\end{tabular}

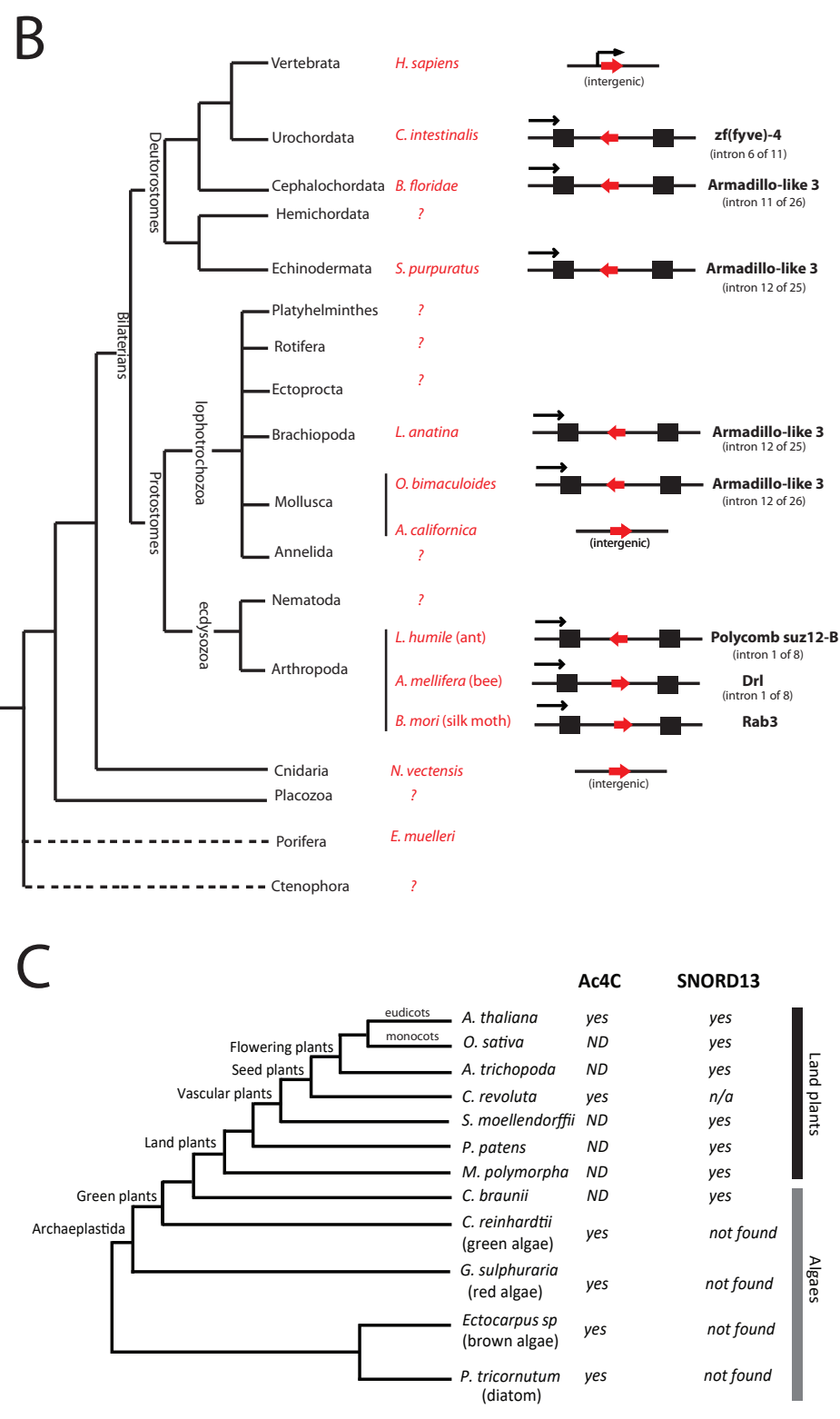


bioRxiv preprint doi: https://doi.org/10.1101/2021.11.30.470322; this version posted November 30, 2021. The copyright holder for this preprint (which was not certified by peer review) is the author/funder. All rights reserved. No reuse allowed without permission.

A

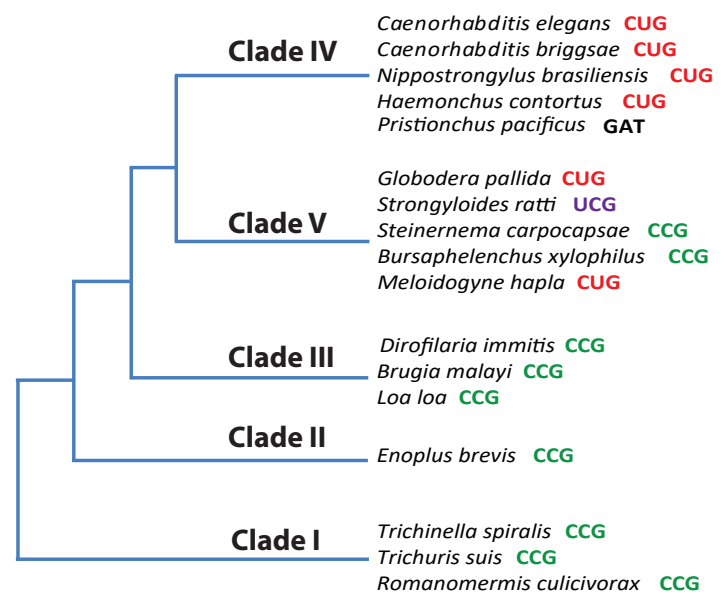

B

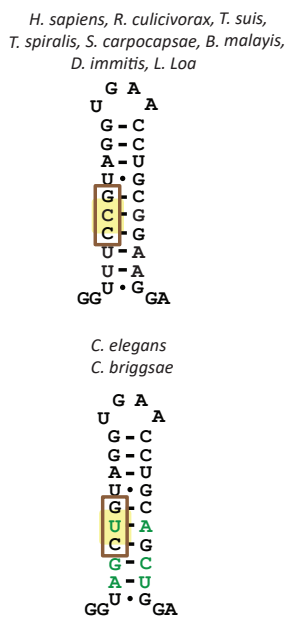

C
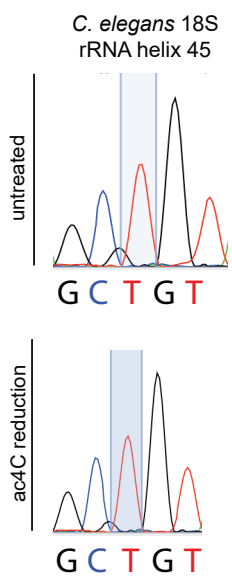

$\mathrm{E}$
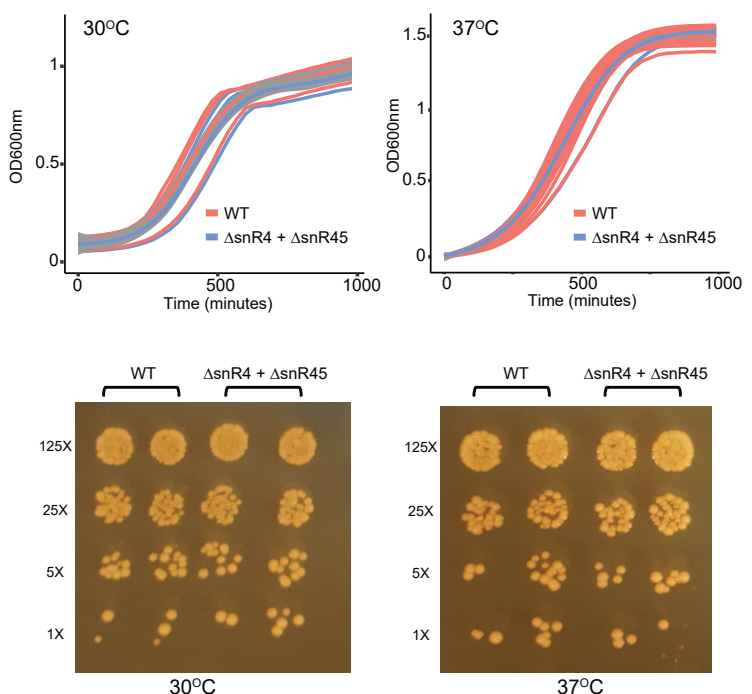
A

SNORD

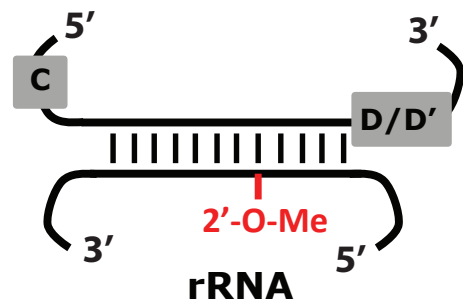

C

SNORD13 (H. sapiens)

SNORD45 (S. cerevisiae)

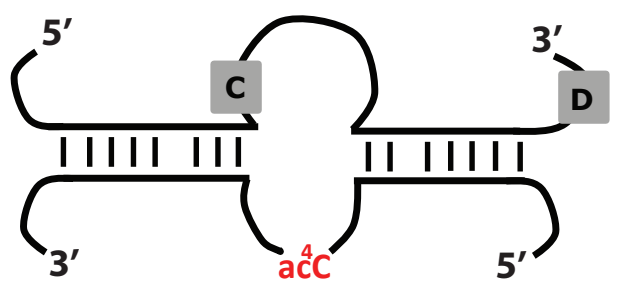

rRNA
B

\section{SNORA}

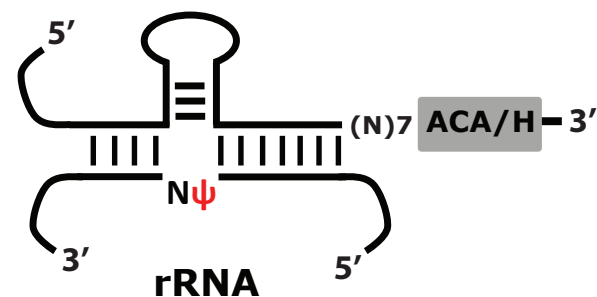

D

SNORD13/Or-CD1

(D. melanogaster)

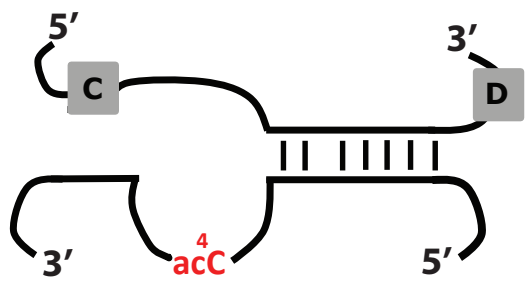

rRNA 
B. floridae GCAUUGUŨC

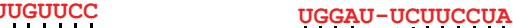

5' -AGUC'CGUA' ' ' ' ' ' ' ' '

\section{C. intestinalis}

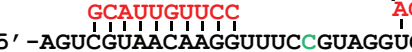

\section{S. purpuratus}

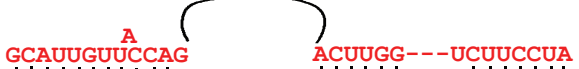

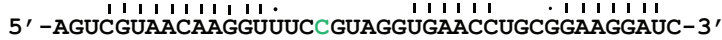

\section{A. californica}

GCAUUGU UCCAA

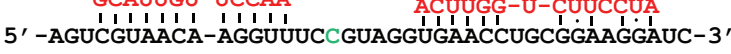

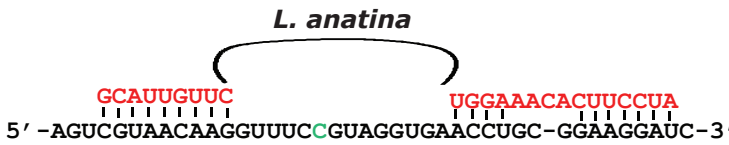

\section{N. vectensis}

GCAUUGUUCC

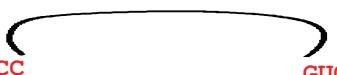

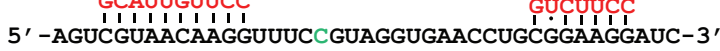

\section{E. muelleri}

GCAUUGUUUUA

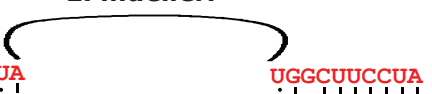

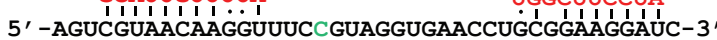

o. sativa

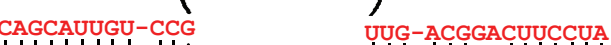

5' -AGUC' ' ' ' ' ' ' ' I' ' '.

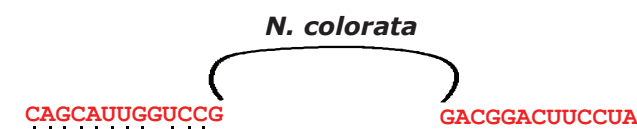

5' -A' ' ' ' ' ' ' ' ' ' ' '

\section{A. trichopoda}

\section{CAGCAUUGGUCCG}

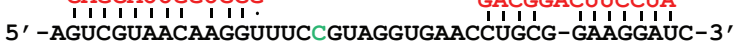

\section{S. moellendorffii}

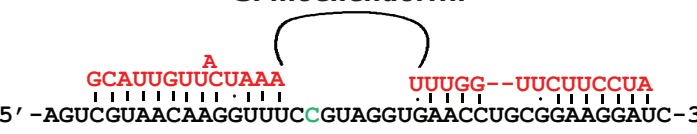

C' ' ' ' ' ' ' ' ' ' ' ' ' '

\section{M. polymorpha}

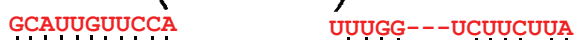

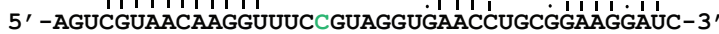

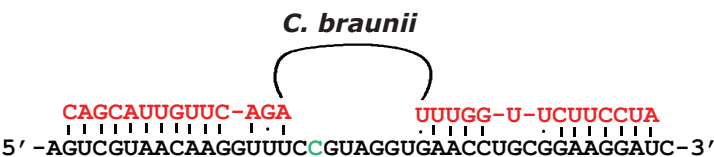

\section{D. melanogaster}

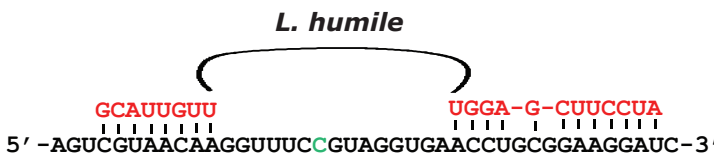

A. mellifera
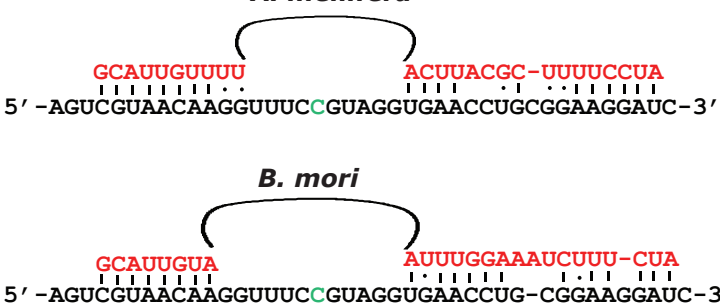


A pools $\begin{array}{ccc}\text { KO } & \text { WT } & \text { B } \\ \text { clones } & \text { clones }\end{array}$

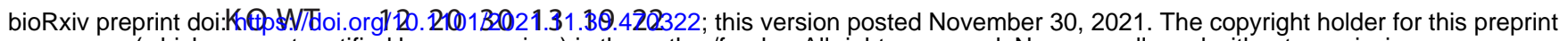
(which was not certified by peer review) is the author/funder. All rights reserved. No reuse allowed without permajssion.

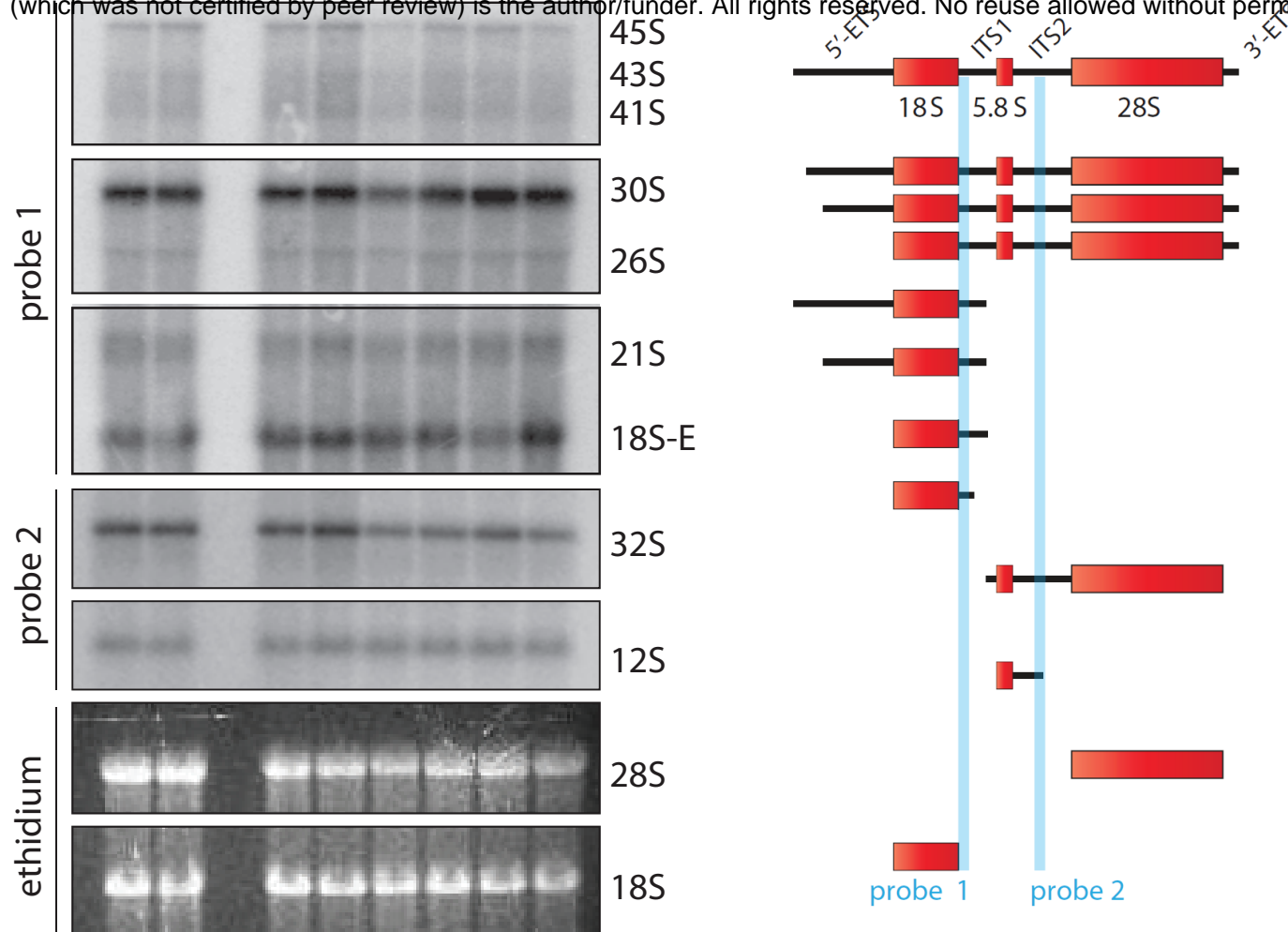

C

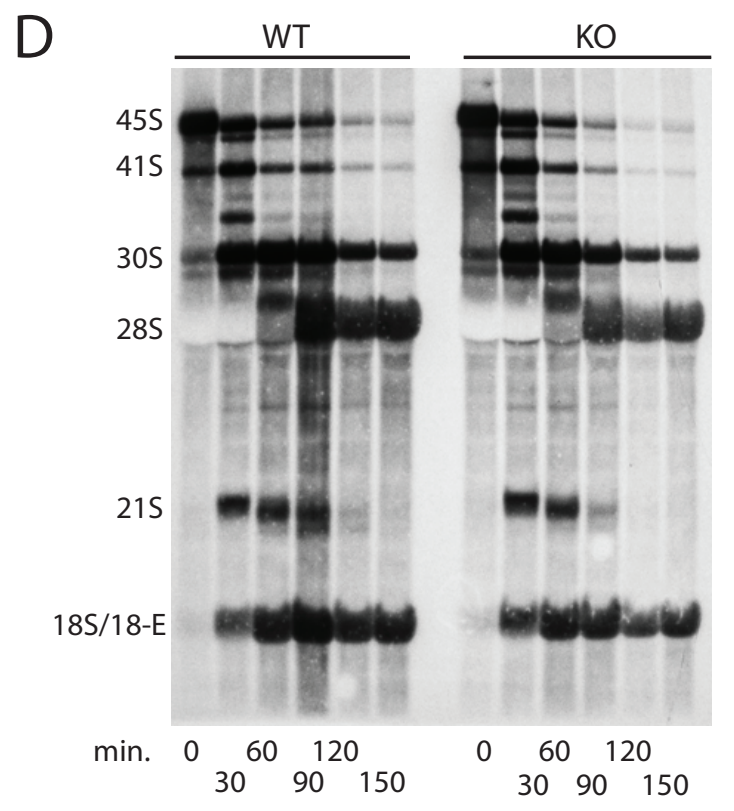

$E$
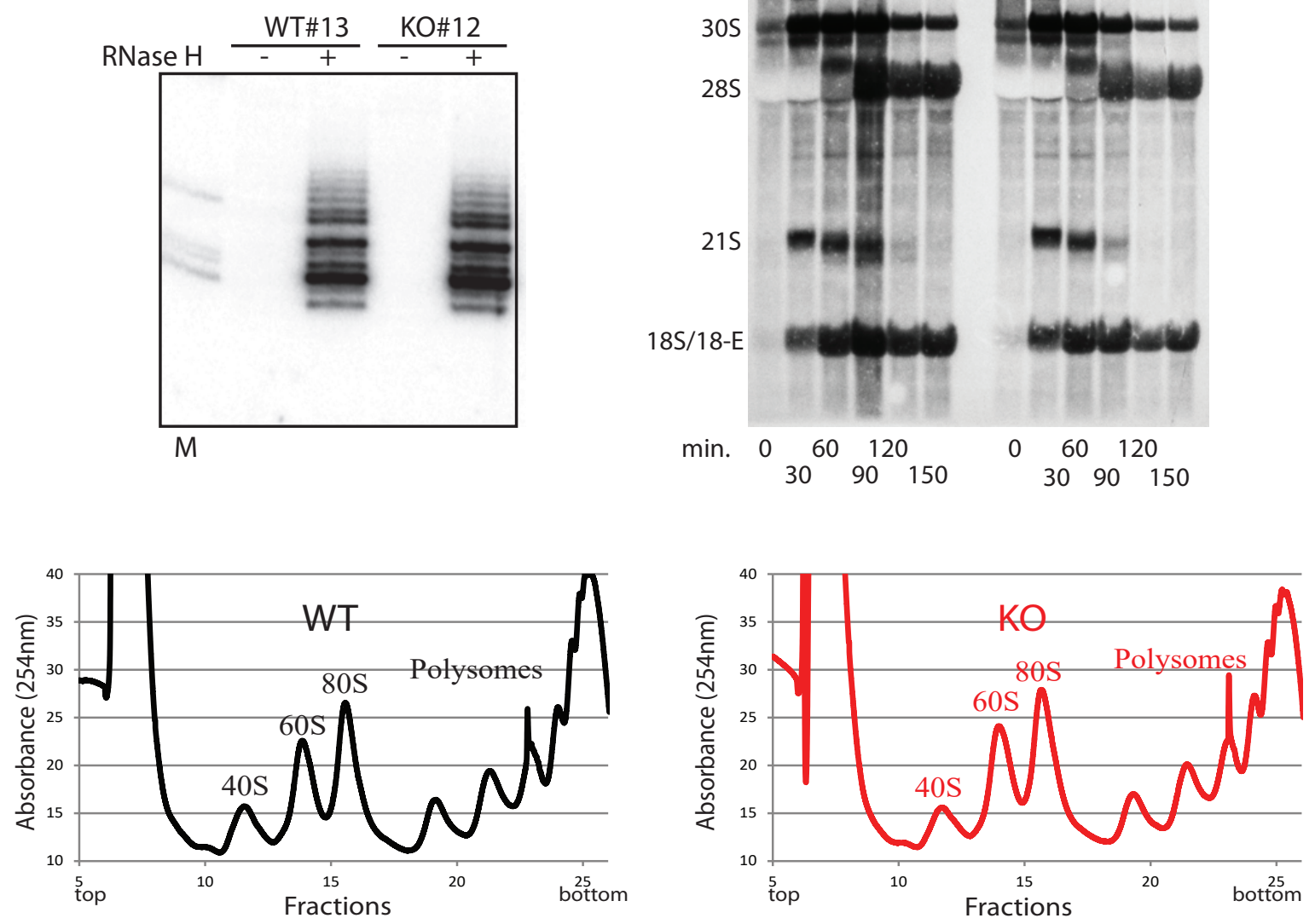
bioRxiv preprint doi: https://doi.org/10.1101/2021.11.30.470322; this version posted November 30, 2021. The copyright holder for this preprint (which was not certified by peer review) is the author/funder. All rights reserved. No reuse allowed without permission.

A

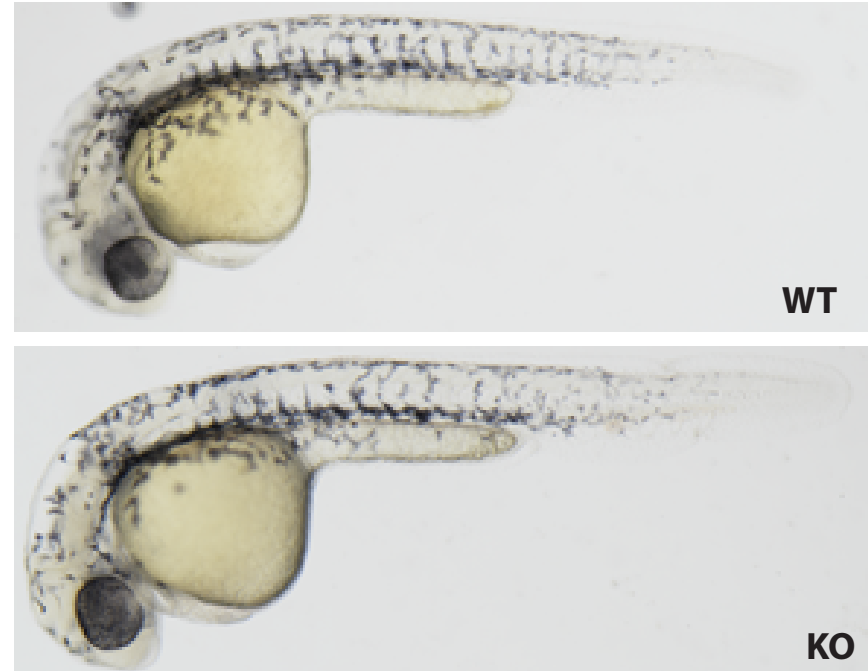

B

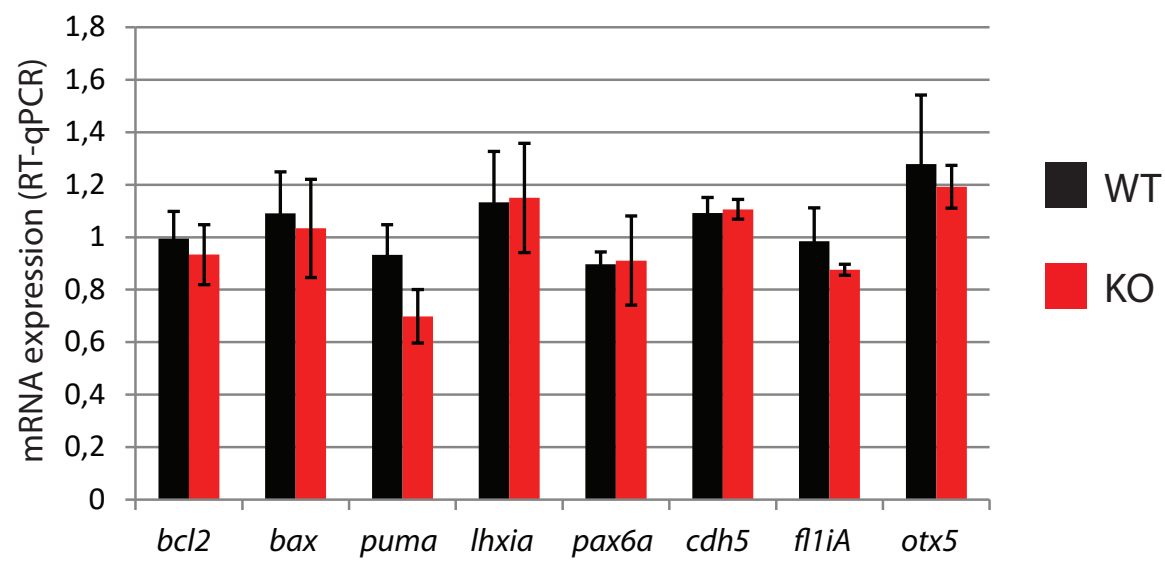




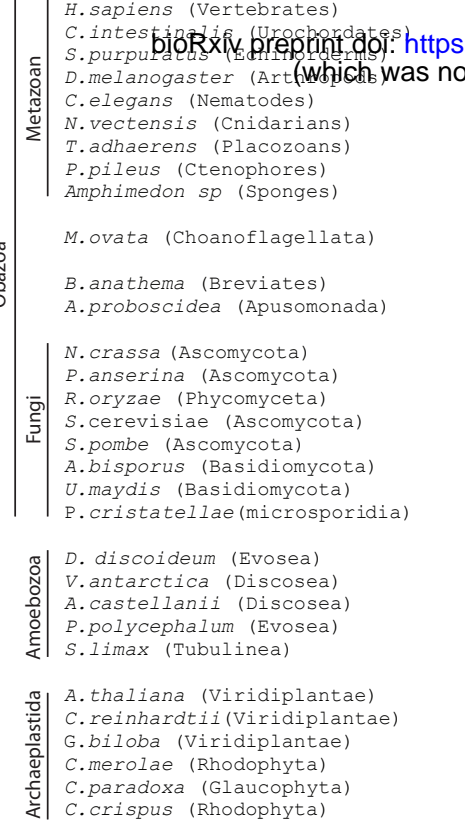

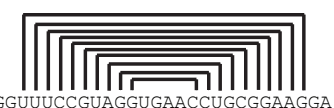

E.siliculosus (Stramenopila)

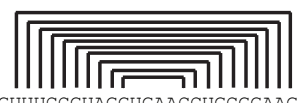

:

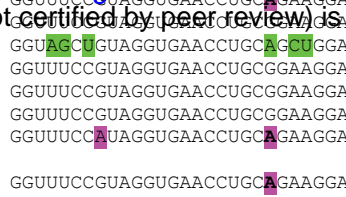

GUUDCGUAGUGAACCUGCA AAAGG

GGUUUCCGUAGGUGAACCUGCGGAAGG

GGUCUCCGUÜGGUGAACCĀGCGGAGGGA

GGUUUCCGUAGGUGAACCUGCGGAAGGA

GGUUUCCGUAGGUGAACCUGCGGAAGGA

GGUUUCCGUAGGUGAACCUGCGGAAGGA

GGUAUCUGUAGGUGAACCUGCAGAUGGA

GGCUGCUAUAGGUGAACCUGUGGCAGGA

GGUAUCCGUAGGUGAACCUGCÄGAAGGA

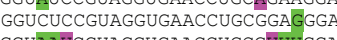

GGUCUCCGUAGGUGAACCUGCGGAGGGA

GGUUUCCGUAGGUGAACCUGCGGAAGGA

GGUUUCCGUAGGUGAACCUGCGGAAGGA GGUUUCCGUAGGUGAACCUGCGGAAGGA GGUUUŪCGUAGGUGAACCUĀCGAAAGGA GGUUUCCGUAGGUGAACCUGCGGAAGG GGUUUCCGUAGGUGAACCUGCAAGAAGA AC4C

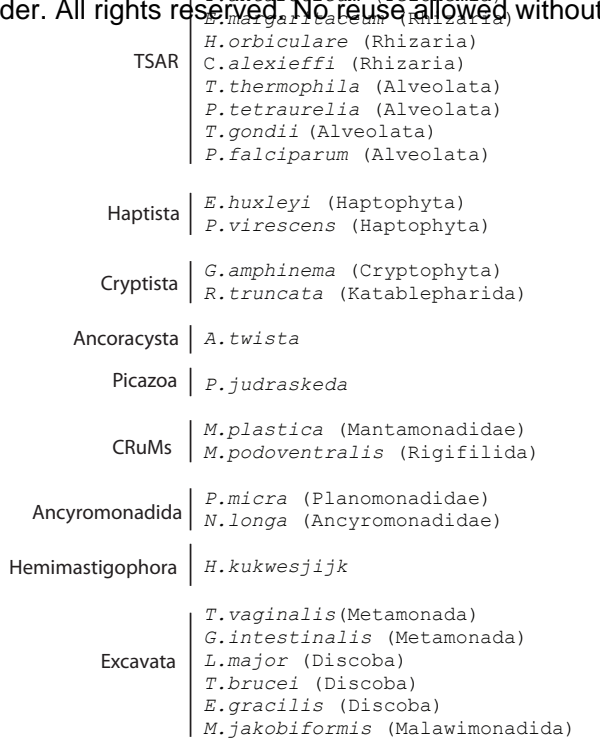

missiona GGCAUCGGUAGGUGAACCUGCCGAUGGA GGUAUCUGUAGGUGAACCUGCAGADGGA GGUUUCCGUAGGUGAACCUGCA GAAGGA GUUUCCGUAGGUGAACCUGCGGAAGG

GGUUUCCGUAGGUGAACCUGCGGAAGGA GGUUUCCGUAGGUGAACCUGCA GAAGGA GGUUUCCGUAGGUGAACCUGCÄGAAGG GGUUUCCGUAGGUGAACCUGCGGAAGGA GGUUUCCGUAGGUGAACCUGCGGAAGGA GGUÄUCCGUAGGUGAACCUGCGGAUUGGA GGUUUCCGUAGGUGAACCUGCGGAAGG

GGUUUCCGUAGGUGAACCUGCGGAAGG GGUUUCCGUAGGUGAACCUGCGGAAGGA

GGUUUCCGUAGGUGAACCUGCGGAAGGA

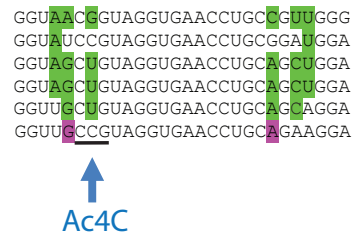

B

H. sapiens, S. pombe

S. purpuratus, D. discoideum,

G. intestinalis (Excavata, Metamonada)

N. crassa, P. anserina

(Fungi)

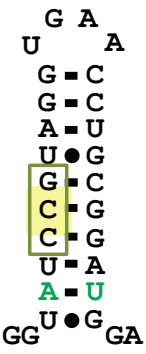

E. gracilis (Excavata, Discoba)

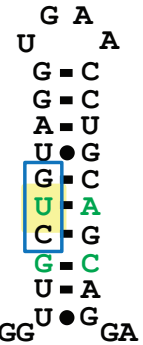

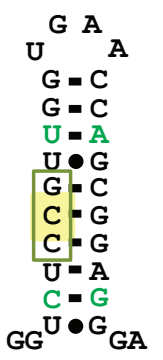

T. brucei, L. major (Excavata, Discoba)

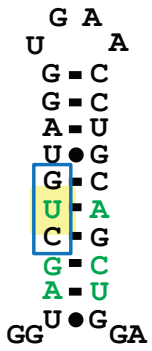

B. daphniae (Fungi, microsporidia)

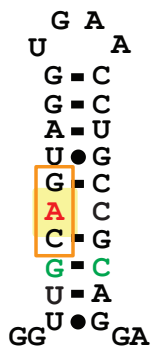

P. polycephalum
(Amoeboza)



S. silvae (Fungi, budding yeast)

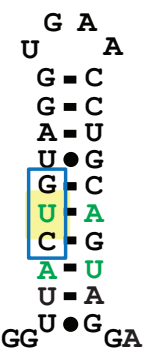

P. cristatellae (Fungi, microsporidia)

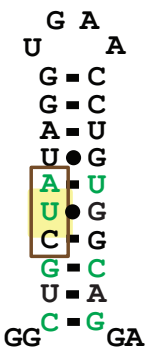

S. robustus (Nematoda)

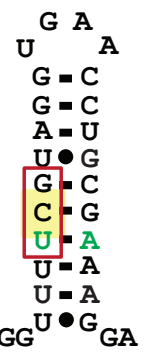

A. tamaren (TSAR, Dinoflagellata)

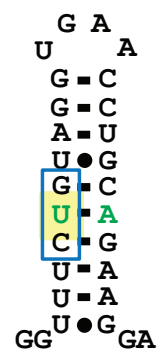

T. vaginalis
(Excavata, Metamonada)

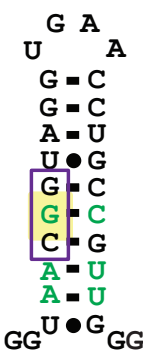

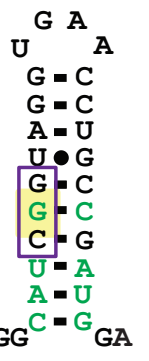

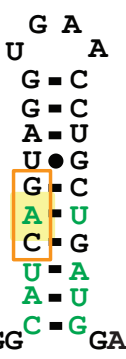

Amphimedon sp.

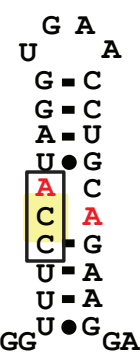


bioRxiv preprint doi: https://doi.org/10.1101/2021.11.30.470322; this version posted November 30, 2021. The copyright holder for this preprint (which was not certified by peer review) is the author/funder. All rights reserved. No reuse allowed without permission.

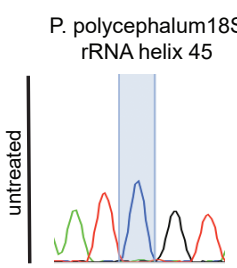

A T C G T



P. polycephalum $18 \mathrm{~S}$

rRNA helix 34

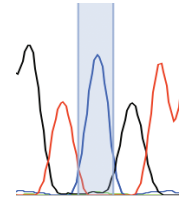

G T C G T

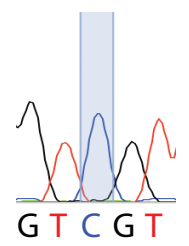

T. thermophila $18 \mathrm{~S}$

rRNA helix 45

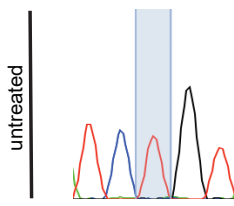

T C T G T

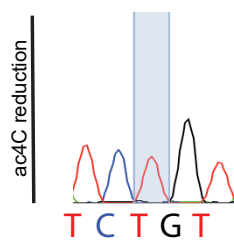

T. thermophila $18 \mathrm{~S}$

rRNA helix 34

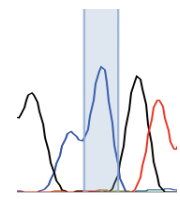

G C C G T

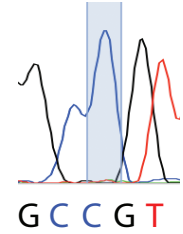

S. carpocapsae 18S

rRNA helix 45

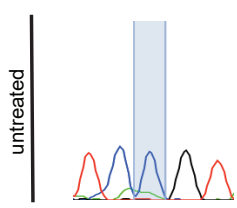

$\mathrm{T}$ C C G T

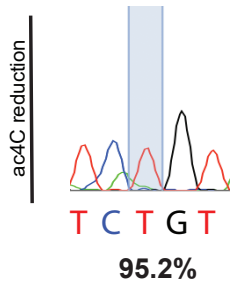


bioRxiv preprint doi: https://doi.org/10.1101/2021.11.30.470322; this version posted November 30, 2021. The copyright holder for this preprint (which was not certified by peer review) is the author/funder. All rights reserved. No reuse allowed without permission.

\section{H. sapiens}

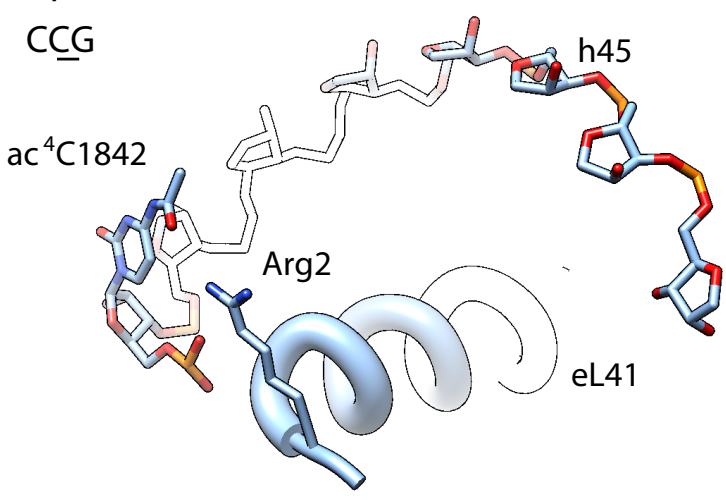

P. falciparum

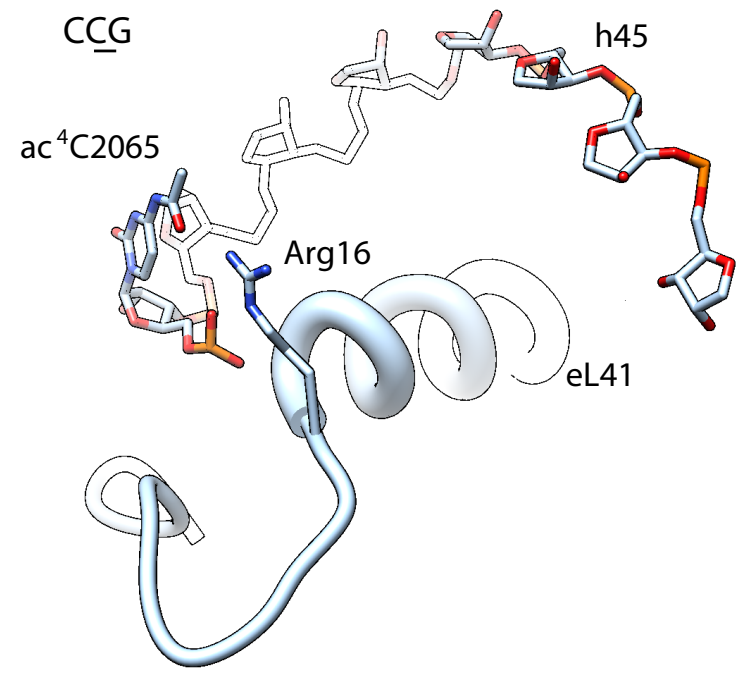

\section{T. gondii}



T. vaginalis

CGG

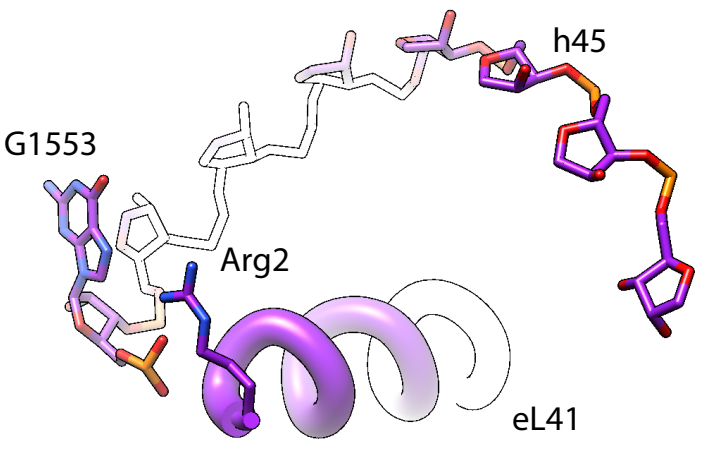

L. donovani

CUGG

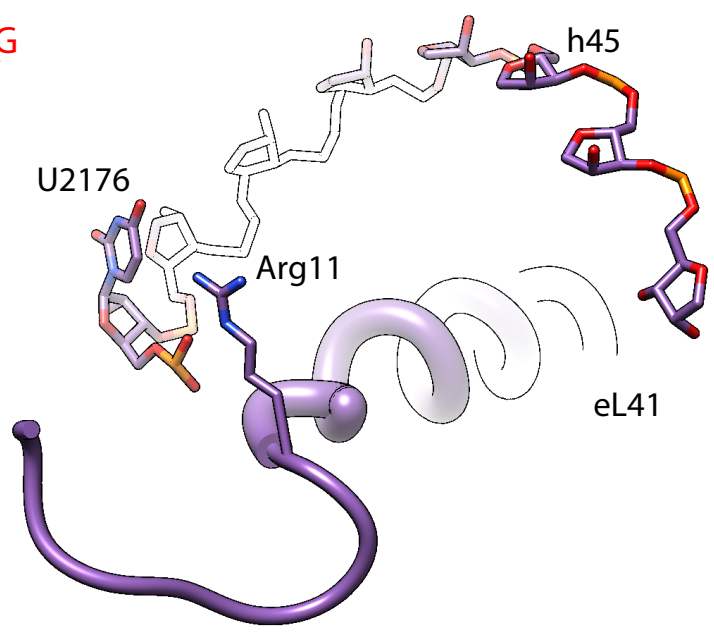

E. gracilis

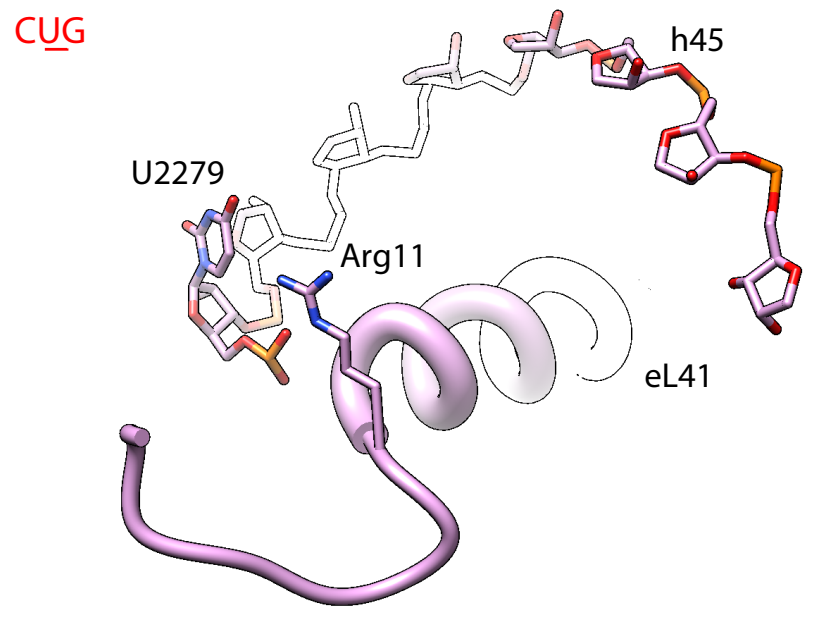


bioRxiv preprint doi: https://doi.org/10.1101/2021.11.30.470322; this version posted November 30, 2021. The copyright holder for this preprint (which was not certified by peer review) is the author/funder. All rights reserved. No reuse allowed without permission.

\section{Supplementary data S1}

\section{- Vertebrata - A few representative species are listed}

>Homo_sapiens

GATCCTTTTGTAGTTCATGAGCGTGATGATTGGGTGTTCATACGCTTGTGTGAGATGTGC

CACCCTTGAACCTTGTTACGACGTGGGCACATTACCCGTCTGACC

$>$ Callithrix_jacchus (monkey)

GATCCTTTTGTAGTTCATGAGCGTGATGATTGGGTGTTCATACGCTTGTGTGAGATGTGC

CACCCTTGAACCTTGTTACGACGTGGGCACATTACCCGTCTGACC

$>$ Mus musculus (mouse)

GATCCTTTCTGGTTCATAAAGCGTGATGATTGGGTGTTCACGCCATTGCGTGACATGTGC CGCCCATAAACCTTGTTACGACGTGGGCACATTACCCGTCTGACA

$>$ Oryctolagus_cuniculus (rabbit)

GATCCTTTTCGTAGTTCATAAGCGTGATGATTGGGTTGTTCACGCAATTGCGTGAGATGT GCCACCCTCGAAACCTTGTTACGACGCCGGCACATGACCCGTCTGACG

$>$ Erinaceus_europaeus (hedgehog)

GATCCTTTTGTAGTTCATAAGTGTGATGATTGGGTTGTCATGCGACTGCGTGAGGTGTGC CACCCATAAACCTTGTTACGACGTCGGCACATTACCCGTCTGACA

$>$ Tursiops_truncatus (dolphin)

GATCCTTTTGTAGTTCATAAGTGTGATGATTGGGTGTTCACGCGCTTGTGTGACATGTGC TTCCTTCCAACCTTGTTACGACTGTGGCACATTACCCGTCTGACA

$>$ Ceratotherium_simum (Rhinoceros)

GATCCTTTTGTAGTTCATAAGCATGATGATTGGGTGTTCACGCGTCTGTGTGAGATGTGC

CTCCCACAAACATTGTTACGACGTCGGCACATCACCCGTCTGACG

$>$ Canis_lupus_familiaris (dog)

GATCCTTTTGTAGTTCATAAGCGTGATGATTGGGTGTTCACGCCGTTGTGTGACATGTGC CTCCCTCAAACCTTGTTACGACGCGGCACATTACCCGTCTGACG

$>$ Pteropus_vampyrus (megabat)

GATCCTTTTGTAGTTCATAAGCGTGATGATCGGGTGTTCATGCTGTTGTGTGAGATGTGC CGCCCATCTAACCTTGTTACGACATTAGGCACATGACCCGTCTGACG

>Loxodonta_africana (Elephant)

GATCCTTTTTCGGTTCATAAGCGTGATGATTGGGTTTTCATACGTCTGTGTGATATGTGC CTCCCCCAAACCTTGTTACGACGTCGGCACATTACCCGTCTGACG

$>$ Dasypus_novemcinctus (Armadillo)

GATCCTTTTGTGGTTCATAAGTATGATGAATGGGTGTTCACGCGCTTGTGTGAGATGTGC CTCCCTCCAACCTTGTTACGACGTGGGCACATTACCCGTCTGACA

$>$ Monodelphis_domestica (Opossum)

GATCCTTCTGGTTCATACAAGTGATGACTGGGTTTTCACGCGTTTGTGTGAGATGTGCCG CCCTTACTTTTCTTGTTACGCATAGGTACATTACCCGTCTGATT

$>$ ornithorhynchus_anatinus (Platipus)

GATCCTTTCGGTTCATTAAAAATGATGATTGGGTTGCCACGCGCCTGCGTGAGATGTGCC GCCCTTTTGTTACGCATGCGCACATTACCCTTCTGATC

$>$ Gallus_gallus (chicken)

GATCCTTCCGGTTCATAGCAAATGATGAATGGGAGTTGCACGCGGCTGCGTGACGTGTGC GCCCTTTGTTACGACGTGCACAGCCCCTTCTGAGC

$>$ Anolis_carolinensis (lizard)

GATCCTTCAGGTTCTTCCATGTGATGAGTGGGTTGTTCATGCGCATGCGTGAGATGTGCC GTCCCAATTGTTACGACAGGGCACAGTACCCATCTGATC

>Xenopus_tropicalis (frog)

GATCCTTCTGGTTCTTACATGTGATGAGTGGGTTATCACACGGCTGTGTGAGATGTGCCT TCCCGACTTGTTACGACTCGGCATAGTACCCATCTGATC

$>$ Takifugu_Rubripes (fugu)

GATCTTTCTGGTTCATGCATGTGATGATTGGTCAAATCACGGATCAATCTGTGAAAGGTG CAGCCTTCTCTTGTTACGCTATGCGCCTCAGCCTCTCTGATC

$>$ Danio_rerio (Zebrafish)

GATCCTTGAGGTTCATACAACATGATGATTGGTCTATCACGACTGTTCGTGAAATGTGCA GCCCTTTGTTACGACGTGGCACATTGACCGTCTGATT

>Petromyzon_marinus (lamprey) 
bioRxiv preprint doi: https://doi.org/10.1101/2021.11.30.470322; this version posted November 30, 2021. The copyright holder for this preprint (which was not certified by peer review) is the author/funder. All rights reserved. No reuse allowed without permission. GATCCTTCCTGGTCATTGAACATGATGAATGGGTCAATCTCGCGAGCTGCGTGAGATGTG

TCTCCCCTTACTCTTGTTACGCAATGGCACATTACCCGTCTGACT

\section{- Urochordata}

$>$ Ciona_intestinalis

GATCCTTCTCAAGGTCAAACGGGCATGATGACGGGTTAATCTCATTGATATATGGGAGATGTGCCGACCCACCCC TTGTTACGCATTGGCGCATAGGACCGTCTGACCCA

\section{- Cephalochordata}

$>$ Branchiostoma_floridae

GATCCTTCTTAGGTCAATGGGTATGATGAGTGGATAGTCATGCCTGTGCGTGACAGGTGTCATCCCCCCATTGTT ACGCAATCGGCACCTGAGCCTTCTGACCTT

$>$ Branchiostoma_belcheri

GATCCTTCTTAGGTCAATAGGTATGATGAGTGGTTCCTCATGCCTGTGCATGACAGGTGTTGTCCCACCATTGTT ACGCATTCAACACCTGAGCCCTCTGACCTT

>Branchiostoma_lanceolatum

GATCCTTCTTAGGTCAATAGGTATGATGAGTGGATACTCATGCCTGTGCGTGACAGGTGTCATCCCCCCATTGTT ACGCAATCGGCACCTGAGCCCCCTGACCTT

\section{- Echinodermata}

>Strongylocentrotus_purpuratus

GATCCTTCTGGTTCAGAACAGATGATGACTTGCAGCATCATGTACCATACATGAGATGTGTCAGCAGACCATTGT TACGCATGGCACATGTGCAATCTGATGCC

>Lytechinus_variegatus

GATCCTTCTGGTTCAGAACAGGTGATGACTTGCATGATCATGTAACTATACATGAGATGTGTCAGCAGACCATTG TTACGCAAGGCACATATGCAATCTGATGCT

>Patiria_miniata

GATCCTTCTAAGGTCAAACGGGAGATGATGATTGGTTGATCATGTCTTAGTACATGAGACGTGTGACCATTTTAT CCTTGTTACATACCATCACGTTGACCATCTGATCCC

$>$ Asterias_rubens

GATCCTTCTAAGGTCAACGGGAGATGATGATTGGTTATTCATGCTCTATACATGAGACATGTCACCATTTATCCT

TGTTACATTCTGACATGAAACCATCTGATCCC

$>$ Anneissia_japonica

GATCCTTCCATAATCATGGAATAATGATGAAAGGTATTTCATGCACCATGCATGAGAGGGGCTGACCTCCCTACT

TGTTACGTGAAGCACCTCACCTATCTGAATCC

$>$ Acanthaster_planci

GATCCTTCTAAGGTCAATTGGGAGGTGATGATTGGTTTATCATGTCCTGTACATGAAACGTGTGCCCATGGTTAC GCACTGACACGTTAGCCATCTGATCCC

\section{- Mollusca}

$>$ Mytilus_galloprovincialis

GGCCTTCCTGGTTTATACATTATGATGAATAGGGTAATCATGTGACTGCATGAGACATG

TCTCCTTCTAACTTGTTACGCAGAAGGCATGTTCCCATCTGAATAA

>Pecten_maximus

GACCTTCTTTACTGATGGTTCATTAAAGAATGATGAATGGGTTTTCAGGCGTCTGTCTGACACATGTCGTCCTCC ATTGTTACGCATGACATGTTACCCAACTGACTCC

$>$ Pomacea_canaliculata

GATCCTTCTGGTTCAAAACGCATGATGAATGGGAAGTCACGCGACTGCGTGATGGTGCCAACCTCCAGTTACGCA ATGGCACATCCCGTCTGACTTG

$>$ Crassostrea_virginica

GTTAATGAAACCTTCTGGTTCTGTCAAATGATGACTGGCTGGGACATGCAACATGCATGACACATGCCACCGACT TGTTACGTTAGGCATGTTTACCCTCTGATTCA

>Aplysia_californica

GATCCTTCTGGTTCAGAAGACGTGATGATCGGGTACTCACGTGGCTACGTGATAGGGTAACCTAATGTTACGCCA AGACCCATAACTGTCTGACTCT 
bioRxiv preprint doi: https://doi.org/10.1101/2021.11.30.470322; this version posted November 30, 2021. The copyright holder for this preprint (which was not certified by peer review) is the author/funder. All rights reserved. No reuse allowed without permission.

$>$ Mizuhopecten_yessoensis

GACCTTCTTTACTGATGGTTCATTTAGAATGATGAAAGGTTTCTCAGGCGTCTGTCTGAGACATGTCACCTCCAT TGTTACGCACGACATGTTACCTCTCTGACTCT

$>0$ ctopus_bimaculoides

GATCCTTCTGTGTTCAGAAGAAATGATGAATCGGGTTATCACGTACTGATATGTGAGACATGTCGCCGTTTACCT TGTTACGACTATACGACATGACACCCTGTCTGATTCT

$>0$ ctopus_sinensis

GATCCTTCTGAGTTCAGAAGAAATGATGAATCGGGTTATCACGTACTGATACGTGAGACATGTCGCCGTTAACCT TGTTACGACATACGACATGACACCCTGTCTGATTCT

$>$ Batillaria_attramentaria

GATCCTTCATGGTTCGAACACATGATGAATGGAGTACATGCGTCTGCATGACAGTGCCAGCCTCCTTGTTACGCT CAGGCACTCAGCCATCTGACTCA

$>$ Crassostrea_gigas

GTTAATGAAACCTTCTGGTTCTGTTACATGATGACTGGTGGGGACATGCTCCATGCATGAGACATGCCGCCGGCT TGTTACGCTAGGCATGTGTACCTTCTGACTCA

>Lottia_gigantean

GGTAATGACCTTCTAGGTCATATGGGATATGATGAACCGGTTAATCTCAGGCATTGTGGAGATATGTGTCAGCCA ACCTTGTTACGCTCTGACACAGAGAACCATCTGATCCC

$>$ Gigantopelta_aegis

GGCCTTTTGGTTCATTAAAAATGATGAATGGGTTATTCATGTGTCTACATGAGATATGTCTCCAGCATTGTTACG CACAGACATATTACCTCTCTGATA

\section{- Brachiopoda}

$>$ Lingula_anatina

GATCCTTCACAAAGGTCATATGGGATGTGATGAACGGGTTAATCATGTTATTACATGAGATGTGCTCCCCTTCAC TTGTTACGCATGAGTACATTAGCCTTCTGATCCC

\section{- Cnidaria}

$>$ Clytia_hemisphaerica

GATCCTTCTGGTTCAACGGAACTGATGAGTCGGTCCTTCACTTTACGTAAGTGATACGTGTTCACGCCAACCTTG TTACGCACGGGCACGTCACCGAGTCTGATCC

$>$ Acropora_millepora

GAATCCTTCAGGATTTAACAAGGGATATGATGAGTGGTTACTTCACTGGTACGTCAGTGAGACGTGTCACCCTTT CGTTACGACACCGACACGAGACCTCTCTGATCCC

$>$ Acropora_digitifera

GAATCCTTCAGGGTTTAACAAGGGATATGATGAGTGGATACTTCACTGGTACGTCAGTGAGACGTGTCACCATTT CGTTACGACACCGACACGAGACCTCTCTGATCCC

$>$ Nematostella_vectensis

GACCTTCTGGTTCAATAGGGAGTTGATGAACGGGTTACTCTCTGTCTGTCAGAGACACGTGCGCCCTTCCTTGTT

ACGCATAGACACGTGACCCTTCTGATCCC

$>$ Pocillopora_damicornis

TATCCTTCTGGTTCATTGAGGAGATGATGATTGGTCCAATCACTGTTCCATCAGTGAGACGTGTCTCCAACTTGT TACGCATAGACACGTGACCTCTCTGATCCT

>Stylophora_pistillata

CATCCTTCTGGTTCATTGAGGAGATGATGATCGGTCCAATCACTGCTCTATCAGTGAGATGTGTCTCCATCTTGT TACGCATAGACACGTGACCTCTCTGATCCT

$>$ Dendronephthya_gigantea

GATTCTTCATTGGTTCATTAGGGAGATGATGATTGGTTAATCACCTCATTAGGTGATAAGTGTCGTCCATTTTTG TTACGCATAGACACTTGCCCATCTGATCCC

>Paramuricea_clavata

TCCTTCATTGGTTCATTAGGGAGATGATGATTGGTTAATCACCTCATTAGGTGAGAAGTGTCGTCATAATTTGTT ACGCATAGACACTTGACCTCTCTGAT

$>$ Exaiptasia_pallida

GRACTTCTGGTTCATGCGGGAAGTGATGAATGGGGTTTTCACTTTTCTTAGTGAGACGTGTCACTCTCCTGTTAC GCACGACACGTAACCCATCTGATCCC

$>$ Actinia_tenebrosa GCCTTCATGGTTCATGCGGGAAGTGATGAACTGGGTAATCGCTAGTATTAGTGAGACGTGTCACCCACCAATGTT ACGCACAGACACGTGACCCTCCTGATCCC 
bioRxiv preprint doi: https://doi.org/10.1101/2021.11.30.470322; this version posted November 30, 2021. The copyright holder for this preprint (which was not certified by peer review) is the author/funder. All rights reserved. No reuse allowed without permission. $>$ Actinia_equinia

GGCCTTCATGGTTCATGCGGGAAGTGATGAACTGGGTAATCACTAGTATTAGTGAGACGTGTCACCCACCAATGT TACGCAAAGACACGTGACCCTCCTGATCCC

\section{- Porifera}

$>$ Ephydatia_muelleri

GATCCTTCGGTTCAAAGTGGAGAGGATGAATGGTTGAGTCACTTGCTATAAGTGAGATGTGCGGCCATTTTGTTA CGCATCGCACAAAGAACCATCTGATCCA

\section{- Arthropoda}

$>$ Lucilia_sericata

AGAAAGTGATGTTTGAGTTCTAACAAACTTTTTTTATGTTTGAGGGCTGTGTGCTCAACC TTTCTATGTTACGACTAATAGCACAACCTTGAAATACAGCTGATTCT

>Stomoxys_calcitrans GGGATATGATGATTGAGTTTCTTGCAGGCATGCTAATGCTTGAGGGCTGTGTTCTCAACA CATAATGTTACGACTGTTAACACAGCTGTGAAATTTATCTGATCCC

>Syritta_pipiens AGGAAGTGAGGATTGGGTTATGTGCTTGCATCGATGCATGATGGCTGTTAGCCCAACAAT TTTTCCTTGTTACGAATTGATAACAGAAGAAAACAATCTGATCCT

$>$ Eristalis_pertinax

GGGAAATGAGGATTGGGGATTGCTTGCTGCTATGCATGAAAGCTGTTGGCTCAACAATTT TTTTTCTTGTTACGATATGTTGACAGAAGAGAACAGTCTGATCCC

>Scaeva_pyrastri

AGGAAATGACGATTGGGTGAAGGACTTGTATTCTATACTTGAAGGCTGTTAGCCCAACAA AATTCTTGTTACGAAATAATAACAGGAGTGAACCATCTGATCCT

$>$ Ceratitis_capitata GGGAAATGAGGATTGGGTTTGTTGCTTGCTTCAGATGCATGATGGCTGTTTACCCAACAA ATATGTTACGACTTTTGAAACAGCCGTGGCATTATCTGATCCC

$>$ Bactrocera_tryoni

GGGAAATGAGGATTGGGTTTGAACTTGCTAACTATGCATGATGGCTGTTTGCCCAACAAT TTTGTTACGACTAATAGAACAGCCGTGGCATTATCTGATCCC

>Bactrocera_oleae GGGAAATGAGGATTGGGTTTGAACTTGCTAACAGTGCGTGAAGGCTGTTTGCCCAAAAGT TTTGTTACGACTAATAGGACAGCCGTGGCATTATCTGATCCC

$>$ Bactrocera_latifrons

GGGAAATGAGGATTGGGTTTGAACTTGCTAGCAATGCGTGAAGGCTGTTTGCCCAACAAT TTTGTTACGACTATTAGAACAGCCGTGGCATTATCTGATCCC

>Rhagoletis_zephyria

GGGAAATGAAGATTGGGTTTGTTCTTGCTTTCACTGCATGAAGGCTGTTCGCCCAACAAT TTTGTTACGACTTATTTGAACAGCCGTGGCATTGTCTGATCCC

$>$ Glossina_austeni

GGGGCGTGAAGATTGTGGTTTGTACTTGCTGTAAGTGCTTGATGGTTGTCTGCCCAAAAC TTAATGTTACGACAAATAAAGACAACCTTGAAACCGTCTGATCCC

$>$ Glossina_pallidipes

GGGGCGTGAAGATTGTGGTTTGTACTTGCTGTAAGTGCTTGATGGTTGTCTGCCCAAAAC TTAATGTTACGACAAATAAAGGCAACCTTGAAACCGTCTGATCCC

$>$ Glossina_morsitans

GGGGCGTGAAGATTGTGGTTTGTACTTGCTTTAAATGCTTGATGGTTGTCTGCCCAAAAC TTAATGTTACGACAAATAAAGGCAACCTTGAAACCGTCTGATCCC

$>$ Glossina_brevipalpis

GGGAAGTGACGATTGTGGCATGTACTTTCTTTAAATGTTTGATGGTTGTCAGCACAAATA

TTAATGTTACGACTGATAAGACAGCCTTGAAACCATCTGATCCC

$>$ Glossina_palpalis

GGGACATGAAGATTGTGGTTTGTACTTGCTGTAAGTGCTTGATGGTTGTCTGCCCAAAAT

TTTATGTTACGACAAATAAAGACAATCTTGAAACCGTCTGATCCC

$>$ Glossina_fuscipes fuscipes

GGGACATGAAGATTGTGGTTTGTACTTGCTGTAAGTGCTTGATGGTTGTCTGCCCAAAAT TTTATGTTACGACAAATAAAGACAATCTTGAAACCGTCTGATCCC 
bioRxiv preprint doi: https://doi.org/10.1101/2021.11.30.470322; this version posted November 30, 2021. The copyright holder for this preprint (which was not certified by peer review) is the author/funder. All rights reserved. No reuse allowed without permission.

$>$ Drosophila_melanogaster

GAGAGGTGACGATTGGGTTCGATCTCGCTATATAATGCTTGAGGGCCTTGTTCTCCCAAC CAATATGTTACGACTTACGGGACAGCCTTGAAAACATCTGATCTC

>Drosophila_ananassae

GAGAGGTGAAGATTGGGCTAGGTCTCGCGAACTGATGCTTGAGGGCCTTGTTCTCCCAAC

CAATATGTTACGACTTACGGAACAGCCTTGAAACGATCTGATCTC

$>$ Drosophila_simulans

GAGAGGTGACGATTGGGTTCGATCTCGCTATAAAATGCTTGAGGGCCTTGTTCTCCCAAC

CAATATGTTACGACTTACGGGACAGCCTTGAAAACATCTGATCTC

$>$ Drosophila_sechellia

CGAGAGGTGACGATTGGGTTCGATCTCGCTATAAAATGCTTGAGGGCCTTGTTCTCCCAA CCAATATGTTACGACTTACGGGACAGCCTTGAAAACATCTGATCTC

$>$ Drosophila_yakuba

GAGAGGTGACGATTGGGTTCGATCTCGCTATATAATGCTTGAGGGCCTTGTTCTCCCAAC CAATATGTTACGACTTACGGGACAGCCTTGAAAACATCTGATCTC

$>$ Drosophila_erecta

GAGAGGTGACGATTGGGTTCGATCTCGCTATATAATGCTTGAGGGCCTTGTTCTCCCAAC CAATATGTTACGACTTACGGGACAGCCTTGAAAACCTCTGATCTC

$>$ Drosophila_pseudoobscura

GAGAAGTGAGGATTGGGCCAGGTCTCGCAATACTATGCTTGAGGGCCTTGTTCTCCCAAC AAATATGTTACGACTTGTGGAACAGCCTTGAAAACATCTGATCTC

$>$ Drosophila_persimilis

GAGAAGTGAGGATTGGGCCAGGTCTCGCAATACTATGCTTGAGGGCCTTGTTCTCCCAAC AAATATGTTACGACTTGTGGAACAGCCTTGAAAACATCTGATCTC

$>$ Drosophila_mojavensis

GAGAGGTGAAGATTGGGTTATTCTTGCCTTAACCATGCTTGAAGGTTCTGTTTGCCCAAC

CAAATATGTTACGATTTACAAAACAGCCTTGAAAACATCTGATCTC

$>$ Drosophila_virilis

GAGAGGTGAAGATTGGGTTACTCTTGCCTTAACCATGCTTGAGGGTCTTGTTCGCCCAAC TTATACGTTACGACTAACAGAACAGCCTTGAGAACATCTGATCTC

$>$ Drosophila_grimshawi

GAGAAGTGAGGATTGGGTTAATCTTGCGTTAACCATGCTTGAGGGCTGTGTTCGCCCAAC ATTTTGTTACGACTAACAGAACAGCCTAGGATAACATCTGATCTC

$>$ D. Willistoni

GAGGTGTGAGGATTGGGTTTAGTTCTAGCATTTTCAATGCTTGATCGCTGTGTTCGCCCA ACTGTTTATGTTACGACTAAAGAACAGCCATGAGAAACATCTGATCTC

$>$ Frankliniella_occidentalis

GATCCTTCTAGGTTTAACGGGAATTGATGAATGGATTATCAAACGATACATTTGTTTGAA GCCTGAGTCCTATCTTGTTACGACTTGTCAGGCGAAACCTATCTGATCCCTC

$>$ Melanaphis_sacchari

GATTCTTCCAGATTCATATGGGACATGATGAACGGGTTTAATCTCTTCTTGAAGAGAAGC CTGTGCCCTCACACTTGTTACGTATTAACAGGTCAAACCTATCTGATCCT

$>$ Diaphorina_citri

GATCCTTCTGTGATTCAGAATGGGATATGATGAATGGGGTATGTCACGTGACTCCGTGAA

GCCTACACTCCTTACCTTGTTACGATTAGGTAGGCGAAACCTCTCTGATCCC

$>$ Cimex_lectularius

GATCCTTCTATGGTATCATATGGGATGTGATGACTGGGTATTCTGATCTTATTCAGGCGC CTGCACCCGTAAAATATGTTACGACTAATACGGCAGGGCAAAACCCAACTGATCCC

$>$ Nilaparvata_lugens

GATCCTTTCAGTTCAATGAAAGATGATGAATAAGGTAAACATCCATGTGGGTGAAACCTG AATCTTTCCTTGTTACGCTTATTCAGGTCAAACCTCTCTGATTTC

$>$ Aphis gossypii

GATTCTTCCAGATTCATATGGGATATGATGAACGGGTCTTATCTCTTCTTATGGAGAATC CTGTGCCTTCAAACTTGTTACGTATTTATCAGGTTAAACCCATCTGATCCCT

$>$ Diuraphis_noxia

GATTCTTCCAGATTCATATGGGACATGATGAATGGGTCTAAACTCTCCTCAAAGAGAAGC CTGAGCCTTCAAACTTGTTACGTATTTAACAGGTCAAACCTATCTGATCCCT

$>$ Myzus_persicae

GATTCTTCCAGATTCATATGGGAAATGATGAACGGGTCTAATCTCTTCTCAAAGAGAAGC CTGTGCCCTCAAACTTGTTACGTATTAACAGGTCAAACCTTTCTGATCCC 
bioRxiv preprint doi: https://doi.org/10.1101/2021.11.30.470322; this version posted November 30, 2021. The copyright holder for this preprint (which was not certified by peer review) is the author/funder. All rights reserved. No reuse allowed without permission.

>acyrthosiphon_pisum

GATTCTTCCAGATTCATATGGGACATGATGAACGGGTGTAAACTCTTCTCAAAGAGAAGC CTGTACCTTCAACCTTGTTACGTATTAACAGGTCAAACCCATCTGATCCC

$>$ Bemisia_tabaci

GATTCTTCTGAGTTCATTGGGAAATGATGATCGGTCATTTATGACTCCTACTGATGACGC CTGAAGCCATTTGTTACGACTTGGTCAGGTGAAACCCATCTGATCCC

$>$ Sipha_flava

GATTCTTCCAGATTCATATGGGACATGATGAACGGGTTTAAACTTCATTCGTGAAGAATC

CTGTGCCTTTAAACTTGTTACGATTTCATCAGGTCAAACCTATCTGATCCC

$>$ Gerris_buenoi

ATGTAGATATTTGTTTGTATGGGGATATGATGATTGGTTGTAACGCGTGTTCTGCGCGAA

GCCTGGACCTCTTTATTAACTTGTTACGATTAAACCCAGGTTAAATACCATCTGATCCT

$>$ Laodelphax_striatellus

GATCCTTTCAGTTCAATGAAAGATGATGAATAAGGTAATCATCCTTCATGGGTGAAGCCT

GAGTCTTTACTTGTTACGCATATTCAGGTCAAACCTCTCTGATTTC

$>$ Ixodes_scapularis

GATTCTTCCGAGATTCAATGGGGAGTGATGAGTCGTGGGTGTTCATGCCAATGCGTGATA CGTGCGACGCCCTGATTGTTACGACGATTGCACGTGACCCCTCTGACCCC

$>$ Varroa_destructor

GATCCTTCTGACCAGTTCAGAAGCATGATGAGTCGGGAGTCATACCTTCCATGGTGACAC GTGTGACGCTATAACCTTGTTACGCATTCGCACGGGACCCGCCTGATTCT

$>$ Varroa_jacobsoni

GATCCTTCTGACCAGTTCAGAAGCATGATGAGTCGGGAGTCATACCTTCCATGGTGACAC GTGTGACGCTATAACCTTGTTACGCATTCGCACGGGACCCGCCTGATTCT

>Dermacentor_silvarum

GATCCTTTAACGGTTCTACTGGGGAGTGATGAGTTGTGGGTTCGCACGCCTCATGCGTGA

TACGTGCCACGCCCTTTTAACCTTGTTACGACGACAGGCACGTAGCCCTTCTGACCCC

$>$ Galendromus_occidentalis )

GATCCTTCTGACTAGATTCAGAAGCATGATGAGTCGGGATTCTGACACCGCAGTGGTGAC ACGTGTGACGCCAACTCCTTGTTACGCAAACGCACGCGACCCGCCTGATTCTCG

$>$ Rhipicephalus_sanguineus

GATCCTTTTTGGTTCTACTGGGGAATGATGAGCTGTGGGTTCGCACGCCTCATGCGTGAT ACGTGCCACGCCCTTTTACCTTGTTACGACGATTGGCACGTAGCCCTTCTGACCCC

$>$ Rhipicephalus_microplus

GATCTGTTGCGGTTCTACTGGGGAATGATGAGTTGTGGGTCTGCATGCCTCGTGCGTGAT

ACGTGCCGCGTCCTTTTACCTTGTTGCGACGATTGGCACGTAGCCCTTCTGACCCC

>Stegodyphus_mimosarum

GATCCTTTCACAGATCAATGGGATGTGATGAGTCGGGTCTCATGCAATTGCATGACACGT GCGACGCTGATCTTGTTACGCACAGTGCACGAGAAAAATCTGATCCC

$>$ Stegodyphus_dumicola

GATCCTTTCACAGATCAATGGGATaTGATGAGTCGGaTCTCATGCAAgTGCATGACACGT

GCGACGCTIGATgTTGTTACGCACAGTGCACGAGAAAAgTCTGATCCC

$>$ Loxosceles_reclusa

GATCCTTTCTGGTTCAAAGGGAAGTGATGAGTCGGTCTTCACGTGATATACGTGAAACGG

GTACCGCTGTTTGTTACGACGTTTGCCCGTAAGCCGTCTGATCCC

>Zootermopsis_nevadensis

GATCCTTCTAAGGTAAAATGGGAGATGATGATTTGGAAAACCACATAATTATATGTGAAG

CCTGAACTGTTATTGTTACGAACACTCAGGTTAATTCCATCTGATCCC

$>$ Blattella_germanica

GATCCTCAAGGTTCAAATGGGAAATGATGAATTGGATATCACGCTTGTGTGTGAAGCCTG

TAACATCTTGTTACGCAAACTCAGGCTGGAAATATCTGATCCC

$>$ Centruroides_sculpturatus

GATCCTTTTGCATTTTCACAAGTAATGGGAAATGATGAGTTGGGGTTCACGATTCTTCGT

GACACGTGCGCCACCTTCTGTTACGCATTGCACGTGACCTTCTGATCCC

$>$ ostrinia_furnacalis

GATCTTTCCAAAGGTTAAATGGGGGATGATGATCGGATTGGACACGCGACTGCGTGACGT CCGCCGCCGACTGTTACGCTTGGGCGGGCCAAAATCCTACTGACTCC

$>$ Vanessa_tameamea

GATCTTTCTAAAGGTTACAACGGGGAATGATGATCGGGCTAATCATGCGTCTGCGTGAAG

TCCGCCACCGATTGTTACGCATTCAGGCGGGCCAAAATCCTTCTGACCCC 
bioRxiv preprint doi: https://doi.org/10.1101/2021.11.30.470322; this version posted November 30, 2021. The copyright holder for this preprint (which was not certified by peer review) is the author/funder. All rights reserved. No reuse allowed without permission.

$>$ Trichoplusia_ni

GATCTTTCCAAAGGTTAAACGGGGGATGATGATCGGATTGGACTTGCGTCTGCATGATGT CCACCACCGAATGTTACGCTTTGGTGGGCTAAAATCCTTCTGACTCC

$>$ Pieris_rapae

TATCCTTTCCCAGGACCAAACGGGGGATGATGATCGGTTTATTCACGCGTCTGCGTGACG TCCGTCACCGATTGTTACGCTTAGACGGGCTAAAAACCTTCTGACCCC

>Papilio_machaon

GATCTTTCCAAAGGTTTAACGGGGAATGATGAATTCGGTTAATCACGCGGCTGCGTGAAG

TCCGTCACCGAATGTTACGCATTTGGCGGGCCTAAACCTTACTGACCCC

>Plutella_xylostella

GATCTTTCCAAAGGTATACGGGGGATGATGATCGGATTGGACATGCGCCTGCATGAAGTC

CGCAGCCGATTGTTACGCTTAGCGGGCTAAAATCCTTCTGACCCC

$>$ Zerene_cesonia

GTATCCTTCCCAGGACCAAACGGGGGATGATGATCGGTTGAATCACGTGTCTACGTGATG TCCGTCACCGAATGTTACGCATTAGACGGGCTAAAACCATTCTGACCCC

$>$ Galleria_mellonella

GATCTTTCTAAAGGTTTTAAATGGAGTATGATGATCGGTTTTTTCACACGTCTGTGTGAT GTCTGCCACCGAAATGTTACGCTTTGGCGGGCTAAATTCCTTCTGACTCC

>Danaus_plexippus

GATCTTTCTAAAGGTTTTAACGGGGAATGATGATCGGGTTAATCACACGTCTGTGTGATG TCCGCTGACCATTGTTACGCAATGGCGGGCTAAAATCCTTCTGACCCC

$>$ Amyelois_transitella

GATCTTTCCAAAGGTTAAACGGGGAATGATGATCGGAATTTTCACGCGACTGCGTGAAGT CCGCAGCCGATTGTTACGCTTGTGCGGGCGAAAATCTTTCTGACCCC

$>$ Hyposmocoma_kahamanoa

GATCTTTCTAAAGGTTAAACGGGGGATGATGATCGGATGGGACACGCGTCTGCGTGATGT

CCGCCACCGAATGTTACGCATTAGGCGGGCCAAAATCCTTCTGACCCC

$>$ Spodoptera_litura

GATCTTTCCAAAGGTTAACGGGGAATGATGATCGGATTGGACATGCGTCTGCATGATGTC

CGTTGCCGATTGTTACGCTTTGACGGGCTAAAATCCTACTGACCCC

$>$ Pararge_aegeria

GATCTTTCTGTAAGGTTTTAAACGGGGGATGATGATTCGGATTGAACACGCGTCTGCGTG AGGTCCGCCGCCGATTGTTACGCATTCGAGCGGGCTAAAATCCTTCTGACCCC

>Bicyclus_anyna

GATCTTTCCAAAGGTTAAACGGGGGATGATGATCCGGGTTTGACACGCGTCTGCGTGATG TCCGCCACCGTACTGTTACGCTTTACGGCGGGCTAAAATCCATCTGACCCC

$>$ Bombyx_mori

GATCTTTCTAAAGGTTTAACGGGATATGATGATCGGATTATTCATGCGACTGCATGATGT TCGTCGCCGAATGTTACGCATAAGGCGGGCTAAAATCCTTCTGATCCC

$>$ Bombyx_mandarina

GATCTTTCTAAAGGTTTAACGGGATATGATGATCGGATTATTCATGCGTCTGCATGATGT

TCGTCGCCGAATGTTACGCATAAGGCGGGCTAAAATCCTTCTGATCCC

$>$ Manduca_sexta

GATCTTTCTAAAGGTTAACGGGGGATGATGATCCGATTAATCACGCGTCTGCGTGAAGTC

CGCCATCGATTGTTACGCATTTGGCGGGCTAAAATCAGACTGACCCC

$>$ Heliconius_melpomene

GATCTTTCTGTAAGGTTTTACGGGGAATGATGATCGGGATGATCACGCGTCTGCGTGAGG

TCCGCCACCGAATTGTTACGCAATACGGCGGGCTCAAATCCTTCTGACCCC

$>$ Melitaea cinxia

GATCTTTCTAAAGGTTAAACGGGGAATGATGATCGGGTTCATCACGCGTCTGCGTGATGT CCGCCACCGATTGTTACGCATTCAGGCGGGCTAAAATCCTTCTGACCCC

$>$ Heliothis_virescens

GATCTTTCCAAAGGTTAACGGGGAATGATGATCGGATTGGACTTGCGATTGCATGATGTC CGTCGCCGAATGTTACGCTTATGGCGGGCTAAAATCCTTCTGACCCC

$>$ Anoplophora_glabripennis

GATCCTTCTGATTGTTCAGATGAGAAGTGATGTTTGGGGTTTTTCAGAAGATTATTTCTG AAGTCTGCTCCCCAGTTTTTTTGTTACGACTTGGAGCAGACGATACTCTTCTGATCTC $>$ Leptinotarsa_decemlineata

(GATCCTTCTGATTGTTCAGATGAGAAGTGATGATTGGGGTTTTCTGGTTAAATACCTGAA

GCCTGTTCCCAATTCTTGTTACGAATTTTGTCAGGCAATACTTTTCTGATCTC

>Diabrotica_virgifera 
bioRxiv preprint doi: https://doi.org/10.1101/2021.11.30.470322; this version posted November 30, 2021. The copyright holder for this preprint (which was not certified by peer review) is the author/funder. All rights reserved. No reuse allowed without permission.

GATCCTTCTGATAGTTCAGATGAGAGATGATGAATGGGTTTTTCAGAAACAAAATTTCTG

AAGTCTGTAACTCCACTTGTTACGATTTGGCAGACGATACCTACCTGATCTC

$>$ Tribolium_castaneum

GATCCTTCTGATGTTCAGATGGGAGATGATGAATGGGTCGTTCAGAAGTCCATTTCTGAG

GCCTGTAGCCCTTTTTGTTACGACTTTAAGCAGGCAATACTTTTCTGATCCC

$>$ Aethina_tumida

GATCCTTCTGATTGTTCAGATGGGAGATGATGAACGGGTTCTTGCAGGGACGTCCCTGAA GCCTGTTCCCAAACTTGTTACGATTTTTGGCAGGCCACTACCCATCTGATCCC

>Sitophilus_oryzae

GATCCTTCTGATCGTTCAGATGGGAGATGATGATTGGGATTTAACAGAATTTTTTCTGAA

GCCTGTACCCATATTGTTACGACTATGGCAGGCAATCCTCATCTGATCCC

$>$ Dendroctonus_ponderosae

GATCCTTTCACAATGGTGAAATTGGAAATGATGAACGGGGACGATCAGATTTTTGTCTGA AGTCTGCACCTATTTGTTACGACTTTTGGCAGACCATCCCTATCTGATCC

$>$ Anoplophora_glabripennis

GATCCTTCTGATTGTTCAGATGAGAAGTGATGTTTGGGGTTTTTCAGAAGATTATTTCTG AAGTCTGCTCCCCAGTTTTTTTGTTACGACTTGGAGCAGACGATACTCTTCTGATCTC $>$ Tigriopus_californicus

GATCCTTCTGAACACAGTTCAAACAGGAATTGATGAATTGGGTTGCATCTTCATAGATGA GGCTTGAGCCAATACTTGTTACGCTCATCAAGCTATACCTATCTGATCCT

$>$ Catajapyx_aquilonaris

GATCCTTCTAAAGTTCAAATGGGAAGTGATGAGCTGGTTTTCTTACCACCATGGTGAGGC CTGCACCACAAAATATACGACACTTGCAGGATAAACCTATCTGATCCC

$>$ Cyphomyrmex_costatus

GATCCTTCAATCAGGTATCAAAATGGGAGATGATGAATGGGTTCACTCAAATTTGTTTGA GACGCCTGCACCCTACTTGTTACGACGAAAGCAGGTTAAACCCCTCTGATCCC

$>$ Dinoponera_quadriceps

GATCCTTCTTAAGTTCGAATGGGAAATGATGAATGGGTTTTGCTCAAATTGTTTGAGACG CCTGAACCTTACTTTGATACGACATTCGGCAGGCTAAACCTCTCTGATCCC

$>$ Nylanderia_fulva

GATCCTTCATTCAGGTATCAAATGGGATGTGATGAATGGGTTCAACTCGAAATTATTCGA GACGCCTGCGTCCCGCTATGTTACGACACTAGCAGGTTAAACCTCTCTGATCCC

$>$ odontomachus_brunneus

GATCCTTCTTGAGTTCGAATGGGAAATGATGAATAGGTTTGGCTCAAATTATTTGAGATG CCTGCATCTTACTTTGTTACGACTTCCAGCAGGCTAAACCTCTCTGATCCC

$>$ Pseudomyrmex_gracilis

TATCCTTCGCGGTTTTAAATTGGGAGATAATGAATGGGTTTATTTCAAATTTTTATTTGA

GACACCTGCACCCTGTATTGTTACGACATTTGCAGGTAAAACCTGTCTGATCC

$>$ Trachymyrmex_cornetzi

GATCCTTCAATCAGGTATCAAAATGGGATGTGATGAATGGGTTTACTCAAATTTGTTTGA

GACGCCTGCACCCTATTTGTTACGACGCAAGCAGGTTAAACCTCTCTGATCCC

$>$ Temnothorax_curvispinosus

GATCCTTCAATCAGGTATCAAATGGGAGGTGATGAACGGGTTTGACTCAAAATTATTTGA

GACGCCTGCAACCCTAGTTGTTACGACGCTAGCAGGTTAAACCCCTCTGATCCC

$>$ Trachymyrmex_septentrionalis

GATCCTTCAATCAGGTATCAAAATGGGACGTGATGAATGGGTTCACTCAAATTTATTTGA

GACGCCTGCACCCTCCTTGTTACGACGCAAGCAGGTAAAACCCCTCTGATCCC

$>$ Trachymyrmex_zeteki

GATCCTTCAATCAGGTATCAAAATGGGATATGATGAATGGGTTTACTCAAATTTGTTTGA GACGCCTGCACCCTACTTGTTGTTACGACCCAAGCAGGTTAAACCTCTCTGATCCC

$>$ Vollenhovia_emeryi

GATCCTTCATTCAGGTATCAAAATGGGAAGTGATGACTGGGTTTAAGCTCAAGATTATTT

GAGACTCCTGCGACCCGTAATTGTTACGACTATGCAGGCTAAACCTCTCTGATCCC

$>$ Linepithema_humile

GATCCTTCGAGGTATCAAATGGGAGATGATGAACGGGTTTAACTCGAATTTTTCGAGACG

CCTGCCACCCTGTTGTTACGACGACAGCAGGTTAAACTCCTCTGATCCC

$>$ ooceraea_biroi

GATCCTTCATTGTCAGGAATCAAACTGGGATATGATGAATAGGTTTACCTCAAATTGTTT

GAGACGCCTGTAACCTTTTTTAACGTTACGACATCGGCAGGCTAAACCGCTCTGATCCC

$>$ Camponotus_floridanus

GATCCTTCATTCAGTTTCAAATGGGAAATGATGAATGGGTTTTACTCAAAATTATTTGAG 
bioRxiv preprint doi: https://doi.org/10.1101/2021.11.30.470322; this version posted November 30, 2021. The copyright holder for this preprint (which was not certified by peer review) is the author/funder. All rights reserved. No reuse allowed without permission.

ACGCCTGCATCCCTTACTTTGTTACGACACTAGCAGGCAAAACCTCTCTGATCCC

$>$ Harpegnathos_saltator

GATCCTTCTTCAGTTCGAATGGGAAATGATGAATAGGTTGGCTCAAGTTATTTGAGACGC

CTGCACCTTACTTTGTTACGACTTTCCTGCAGGTTAAACCTCTCTGATCCC

$>$ Wasmannia_auropunctata

GATCCTTCGATCAGGTATCAAAATGGGAGgTGATGAACGGGTTTTGACTCAAAATTGTTT

GAGACGCCTGCACCCTAGTTGTTACGACGTTAGCAGGTTAAACCCCTCTGATCCC

$>$ Acromyrmex_echinatior

GATCCTTCTCTTAGGTATCAAAATGGGATGTGATGAATGGGTTTACTCAAATTTGTTTGA

GACGCCTGCATCCTACTTGTTGTTACGACTCAAGCAGGTAAAACCTCTCTGATCCC

$>$ Monomorium_pharaonis

GATCCTTCTCGAGGTATCAAAATGGGAGATGATGAATGGGTTTGACTCAAAGTTATTTGA

GATGCCTGCACCCTGATTGTTACGACACAAGCAGGCTAAACTCCTCTGATCCC

$>$ Solenopsis_invicta

GATCCTTCTCGAGGTATCAAAAGGGAGATGATGAATGGGTTTATCTCAAAATTATTTGAG

ACGCCTGCACCCTGACTGTTACGACGAGAGCAGGCTAAACCCCTCTGATCCC

$>$ Pogonomyrmex_barbatus

GATCCTTCAATCAGGTATCAAGATGGGAGATGATGAACGGGTTTGACTCAATATTATTTG

AGACGCCTGCACCCTGTTTGTTACGACGCAAGCAGGCTAAACCCATCTGATCCC

$>$ Pogonomyrmex_barbatus

GATCCTTCAATCAGGTATCAAGATGGGAGATGATGAACGGGTTTGACTCAATATTATTTG AGACGCCTGCACCCTGTTTGTTACGACGCAAGCAGGCTAAACCCATCTGATCCC

$>$ Cardiocondyla_obscurior

GATCCTTCTTTAAGGTGAAAATGGGATATGATGAACGGGTTTAGCTCGAATTTGTTTGAG ACGCCTGCAGCCTTTATTGTTACGACGATTGCAGGTTAAACCTCTCTGATCCC

$>$ Bombus_terrestris

GATCCTTCTTTAAGGAAAAATGGGAAATGATGATTAGGTTATCCTCATTTTCATATGAGA AGCCTGCATTCTTACATACTTGTTACGCTTTATGCAGGTCAAACCTCTCTGATCCC

>Bombus_impatiens

GATTCTTCTTTAAGGAAAAATGGGAAATGATGATTAGGTTATTCTCGTTTCCATATGAGA

AGCCTGCATTCTTTCATACTTGTTACGCTTTATGCAGGTTAAACCTCTCTGATCCC

$>$ Cephus_cinctus

GATCCTTCTGTGATTCAGAACGGGGTGTGATGATATGGTTTTGCTCGTTCTGTTACGAGA

CGCCTGCGGCCACAATCCTTGTTACGATTATAAGGCAGGCTAAACCGCTCTGATCCC

$>$ Trichogramma_pretiosum

GATCCTTCTGGATTCAGAACGGGAGATGATGACTGAGTAAGTCGCTCGTTACCCATACGA

GATGTCTGTTCGCTCGGAACCTTGTTACGCAATCGGCAGACTATGACACTCTGATCCC

$>$ Fopius_arisanus

GATCCTTCTGTGATTCAGAACGGGAGATGATGACTCGTTTTGGCTCGCAATTGCGAGATG

CCTGTTACGGCAATCCTTGTTACGATTATACAGGCCAAACCTCTCTGATTCC

$>$ Chelonus_insularis

GATCCTTCGCGATTCAAAACGAGAGATGATGATTCGTTAGGACTCGTTACATACGAGAAG

CCTGCACGGAAACTTGTTACGATTCAGCAGGCTAAACGGCTCTGATCTC

$>$ Neodiprion_lecontei

GATCCTTCTGAGATTCAGAACGGGAAATGATGACGCGGTCGACCCTGTTTCATACGGGAC GCCTGCACCGGCAAACCTTGTTACGATAACGCAGGTCAAACCTCTCTGATCCC

$>$ Orussus_abietinus

GATCCTTCTGTGATTCAGAACGGGGTGTGATGACTGGGTTGTTCTCGTTTTCATACGAGA

CGCCTGCACCGGGATTCTTGTTACGATTCTAGCAGGTCAAACCTCTCTGATCCC

$>$ Diachasma_alloeum

GATCCTTCTGTGATTCAGAACGGGAGATGATGACTCGTTTTGGCTCGCATTTGCGAGATG

CCTGCTACGGCAAACCTTGTTACGATCAAGCAGGCGAAACCTCTCTGATTCC

$>$ Belonocnema_treatae

GATCCTTCTGTGATTCAGATGGGAGATGATGATTTGGTTTAGCTCGTTCGATACGAGAAG

CCTGCGCAACCAAACTTGTTACGACAGTGCAGGCAAAACCGCTCTGATCCC

$>$ Vespa_mandarinia

GATCCTTCTGTTGATCAGAATGGGAAATGATGATCTGGTTTCTCTCGTCGTTACGAGATG

CCTGCACCATATTTGTTACGACTTTTGGCAGGTTGAACCTCTCTGATCCC

$>$ Eufriesea_mexicana

GATCTTTCTAAAGTCAATGgGAAATGATGAAGAGgTTATTCTCATTCTTATATGAGAAGC

CTGCACAAATCTTATACTTGTTACAATTTAAAGCAGGTTAAACCTTTCTGATCCC 
bioRxiv preprint doi: https://doi.org/10.1101/2021.11.30.470322; this version posted November 30, 2021. The copyright holder for this preprint (which was not certified by peer review) is the author/funder. All rights reserved. No reuse allowed without permission. $>$ Megalopta_genalis

GATTCTTCCCAATCAAAAGGGATATGATGACTGGGTGAATCTCGTGCTGATACGATACGC CTGCACCGTTTCATTTCTTGTTACGTTTAAAGCAGGTTAAACCCATCTGATCCC

$>$ Nomia_melanderi

GTATCCTTCTTAGGTGTAAAAGGGATATGATGATTGGGATCTTCTCGTTTTTATACGAGA

TGCCTGCACTTTATACTTGTTACGATTAAAGCAGGTTAAACCTCTCTGATCCC

$>$ Apis_mellifera

CATCCTTTTCGCATTCAATGGGAGATGATGAATATCGTGTTCTCATTCTCGATATGAGAG

GCCTGAAGTTTTTTTTTTGTTACGCACTTTGTCAGGTTATACTATTCTGATCCC

$>$ Apis_florea

CATCCTTCTAGCAGTCAAATGGGATATGATGAATGACGTGTTCTCATTCTAGATATGAGA

GGCCTGAAGTTTTTTTTTTGTTACGACATTTATTCAGGTTATACCATTCTGATCCC

$>$ Apis dorsata

CATCCTTCCTGCGATCAATGGGAGATGATGAATGTCGTATTCTCATTCTCGATATGAGAG

GCCTGAGTTTTTTTTTCTTGTTACGTAGTTTGTCAGGTTATACCATTCTGATCCC

$>$ Apis_cerana

CATCCTTCCGATGTTTAATGGGAGATGATGAATGTCGTGTTCTCATTTTCGATATGAGAG GCCTGAAATTTTTTTTTTTTGTTACGCAATTTGTCAGGTTAAACCATTCTGATCCC

$>$ Dufourea_novaeangliae

GATCTTTCCAGTTCAAATGGGATGTGATGATTGGGTAATTCTCGTTTTTATATGAGACGC CTGCATGCTCTTAATTTTGTTACGCTTAAGCAGGTTAAACCCTTCTGATCCC

$>$ Ceratina_calcarata

GATTCTTCCTGAATTTCAAATGGGAAGTGATGATAAGGTTTTTCTCGTCCGAATACGAGA AGCCTGCGGATTTTTATCCTTGTTACGTTCAACGCAGGTTAAACCTCTCTGATCCC

$>$ Melipona_quadrifasciata

GATTCTTCTGTAAGGAAATGTGGGAAATGATGATCTAGGTTATGCTCATCTTTAATATGA

GAAGCCTGCATTTGTTATACTTGTTACATTTTATGCAGGTTAAACCTCTCTGATCCC

$>$ Habropoda_laboriosa

GTTCATATATTTCTTACATGGGAGGTGATGATTAGGTTACTCTCATCCAGATATGAGAAG

CCTGCTTTTCTAAATTCTTGTTACGCTTTAAAGCAGGTTAAACCTTTCTGATCCC

$>$ Copidosoma_floridanum

GATCCTTCTGGGATTCAGAACGGGAAATGATGACCGAGGTGAATCTCGTTTTGATACGAG

ACGTCTGTGCACTCGAACTTGTTACGCATATGGCAGACTATGACCCTCTGATCCC

$>$ Microplitis_demolitor

GATCCTTCTGTGATTCAGAACAAGAAATGATGACTCGTGAGAGCTCGTCGTATACGAGAT GCCTGCAACGGACATCCTTGTTACGATTTAGCAGGCCAAACATTTCTGATCTT

$>$ Nasonia_vitripennis

GATCCTTCTGTGATTCAGAACGGGATATGATGACTGAGTCTAGCTCGTTCTATACGAGAT GTCTGCGCGCTCGCAAACTTGTTACGCTTACGGCAGACCATGACCCTCTGATCCC

$>$ Polistes_canadensis

GATCCTTCTGTTGATCAGAATGGGACGTGATGATTTGGTTTTGCTCGTCGATACGAGATG

CCTGCACCGACTCTTGTTACGACTTCGGCAGGATAAACCTCTCTGATCCC

$>$ Polistes_dominula

GATCCTTCAAAGGTCATATGGGAAATGATGATTTGGTTTTGCTCGTTGTTACGAGATGCC

TGCACCGACATTTGTTACGACTATTGCAGGTTAAACCTCTCTGATCCC

$>$ Athalia_rosae

GATCCTTCTGAGATTCAGAACGGGAGGTGATGACTCGGGGCGGCTTGTCATTGATACGAG ATGCCTACACCGGCAAACCTTGTTACGATACTGTAGGTCAAACCCCTCTGATCCC

$>$ Megachile_rotundata

GATCCTTCGCGTTCAAAAAGGGATATGATGAATCGGTCTATCTTGTCTTTTACAAGAAGC CTGCACCGTGTTACGACGTGGCAGGTTAAACCTCTCTGATCCC

\section{- Archeaplastida - A few representative species are listed}

>Syntrichia_caninervis

GATCCTTCTGGTTTTACAACATGATGAATGGACAATTCTCGCGTCTGCGTGACAGGTGCC

CGCTTCACATACCTTGTTAAACAGGGCACCCTCCATATCTGACCC

$>$ Physcomitrella_patens

GATCCTTCTGGTTTTACAAAATGATGAGAGGAGGAGTCGCGCGTCTGCGCGAGAGGTGCC 
bioRxiv preprint doi: https://doi.org/10.1101/2021.11.30.470322; this version posted November 30, 2021. The copyright holder for this preprint (which was not certified by peer review) is the author/funder. All rights reserved. No reuse allowed without permission.

CGCCCCAAATACCTTGTTACAAAGGGCAACCCCCCCCTGTCTGACCC

$>$ Marchantia_polymorpha

GATTCTTCTGGTTTTTAAATATGATGAATGGCTTATTCTCGCGCTTGCGAGAGAGGTGTC CGCAGCACCTTGTTACGCATGGACACCGCCTGTTCTGATCCTT

$>$ Amborella_trichopoda

GATCCTTCAGGCAGAACGGGAAGATGAGGAGTGGTATTTTCTCACTTGTGAGATGAGTGT CTGCCCCATGCCTGGTTACGACAAATGGGCACTCACATTCTAATCCTATCTGATTCC

$>$ Nymphea_colorata

GATCCTTCAGGCAGCAAGGGAAGGTGATGAGTGGGTCTAATCTCGCTGATAACATGCGGG ATGGGTGTTCGCCCCAACGCCTGGTTACGACGCTCGGGCACCCCAATCTTAACCATTCTG ATTCC

$>$ Selaginella_moellendorffii

GATCCTTCTTGGTTTTACTGGGGGATGATGAGTGGTAAATGCTCGGTTTCTCCACTGTGA GAGGGCTTGCCCGCCCAAAATCATTGTTACGCAAGGGGCAGTCTCCTATTTTGCGCCTCT GACCT

$>$ Chara_braunii

GATCCTTCTTGGTTTTATGAAATGATGATGAGTGGTGACTCACCTCCCTTTTGCATGTGA GAGGTGGCCCTCACCCAGACTTGTTACGACCGGTCGGCCGCCTAAGCCTATCTGACCCTT $>$ Arabidopsis_thaliana

GATCCTTCAGGCAAGTTAAAGGGGATATGATGAATGGTAAAAACTCGCTTATATTGCGAG AAGAGCGTTCCGCCCAAAGCCTGTTACGACTATTGGGCGCTCTCTTTTTTACACAATCTG ATCCCT

>0ryza_Sativa_Japonica_Group

GATCCTTCAGGCAGTTAAAGGGAGATGATGAGTGGTAACATCTCGCTAATTAATGTTGCG AGAGGGGTGTCTGCCCCAACCAAGCCTGTTACGACAACAGGGCACTCCAATATTTTACCA TCTGATCCC

>Theobroma_cacao_Matina_1.6

GATCCTTCAGGCAGTAAACGGGATATGATGAGTGGTCATTACTCACAAGCGCTGTGAGAT GGGTGTCCGCCCCAACGCCTGGTTACGACAACATGGGCACTCCCCTCTGTACCGCCTGAT CCC

$>$ Arabidopsis_halleri

GATCCTTCAGGCAAGTTAAAGGGGAAATGATGAATGGTAAGTACTCGCTTCTATTGCGAG AAGAGCGTTCCGCCCAAAGCCTGTTACGACAATTGGGCGCTCTCATTTTTACACCGTCTG ATCCC

$>$ Beta_vulgaris

GATCCTTCAGGCAGTAAATGGAATATGAGGAGTGGTCATTTCTCGCTTATATAAATTGCG AGAGGGGTGTCCGCCCAAGCCTGGTTACGACAAATTGGGCATCCCCATTCTTAACCATCT GATTCCT

$>$ Brassica_oleracea

GATCCTTCAGGTTTAAAGGGGAAGTGAGGATTGGGTTTAATCTCGCATATTTTATTGCGT GAAGAGCGTTCCGCTCAAACCTTTTTACGACAATTGGACGCTCCAATTTTCTTATCATCT GATCCC

$>$ Arabidopsis_lyrata

GATCCTTCAGGCAAGTTAAAGGGGAAATGATGAATGGTAAATACTCGCTTCTATTGCGAG AAGAGCGTTCCGCCCAAAGCCTGTTACGACAATTGGGCGCTCTCATTTTTTACACAATCT GATCCC

>Brassica_napus

GATCCTTCAGGTTTAAAGGGGAAGTGAGGATTGGGTTTAATCACGCATATTTAATTGTGT

GAAGAGCGTTCCGCTCAAACCTTTTTACGACAATTGGGCGCTCCAATTTTCATATCATCT GATCCC

>Brassica_rapa

GATCCTTCAGGTTTAAAGGGGAAGTGAGGATTGGGTTATTCTCGCATATATGCGTGAAGA GCGTTCCGCTCAAACCTTTTTACGACAATTGGGCGCTCCAATTTTCAATATATCTGATCC $\mathrm{C}$

$>$ Actinidia_chinensis

GATCCTTCAGGCAGTTTAAAGGGGATGTGATGAGTGGTCAACACTCGCAATAAAAAATGC GAGATGGGTGCTACGGCACAAAGCCTGGTTACGACAAATGCGGGCACTCTCATCTGAACC AATCTGATCCC

$>$ Corchorus_capsularis

GATCCTTCAGGCAGTAAACGGGATATGATGAGTGGTCATTACTCGCACGCGATGCGAGAT GGGTGTCCGCCACGCCTGGTTACGACAACCTGGGCACTCCCATTTTTACCATCTGATCCC 
bioRxiv preprint doi: https://doi.org/10.1101/2021.11.30.470322; this version posted November 30, 2021. The copyright holder for this preprint (which was not certified by peer review) is the author/funder. All rights reserved. No reuse allowed without permission.

$>$ Vigna_radiata

GATCCTTCAGGCAGTTAACGGGATGTGATGAGTGGTAAATGCTTGCATATAACATGCAAG AGGGGTGTCCGCCCCAGCCTGGTTACGACTATGGGGCACTCTCTTTTTGACCTATCTGAT CCC

$>$ Vigna_angularis

GATCCTTCAGGCAGTTAATGGGATATGATGAGTGGTAAATGCTTGCACTTAATATGCAAG AGGGGTGTCCGCCCCAGCCTGGTTACGACTATGAGGCACTCTCTTTTTTACCTATCTGAT CCC

$>$ Cynara_cardunculus

GATCCTTCAGGCAAGTTTTAATGGGAAGTGATGAGTGGTCGCTACTCGCATTTAATATGC GAGAAGGGGTGTTCGCCTAAAGCCTTACGACAATTGGCCACCTCCAATTTTTAACCATCT GATCCCT

$>$ Glycine_max

GATCCTTCAGGCAGTTAACGGGATATGAGGAGTGGTGAAATCTCGCACAAAATATGCGAG TGGGGTGTCCACCCCAGCCTGGTTACGACACTAGGACACTCTCTTTTTAAACCAATCTGAT $\mathrm{CCC}$

$>$ Daucus_carota

GATCCTTCAGGCAGATAATGGGATGTGATGAGCGGTCAGAATATTGCAATAAAAAATGCA ATATGGGTGTCTAGCCCAAGCCTGTTACGACAACATAGGCGCTCTCGTTTTTAACCAATC TGATCCC

$>$ Cucumis_sativus

GATCCTTCAGGCAGTAACGGGATATGATGAGTGGTTGGCTCTCGCTAATATAATGCGAGT

TGGGTGTCCGCCCCAACGCCTGGTTACGACAATTGGGCACTCCCATCTCTTCCAATCTGA

TCCC

$>$ Medicago_truncatula

GATCCTTCAGGCAGAAACGGGATATGATGAGTGGTTGAGTCTCGCACATAATATGCGAGT TGGGTGTTCGCCCCAAGCCTGGTTACGACAATTGGGCACTCTCATTTAATCAATCTGATC $\mathrm{CC}$

>Phaseolus_vulgaris

GATCCTTCAGGTTTATTGGATGTGATGAGTGGTAAAGACTTGCATACAATATGCAAGAGG GGTGTCCGCCCCAGCCTTGTTACGACTGGGGGCACTCAATCTTTAGACCAATCTGATCCT >Lupinus_angustifolius

GATCCTTCAGGCAGTTAACGGGATATGATGAGTGGTTAAATGTCGCAATAAAAAATGCGA GTTGGGTGTTCGCCCAAGCCTGGTTACGACAACTGGGCACCCTCTTATTATTCCAATCTG ATCCC

>Prunus_persica

GATCCTTCAGGCAGAAACGGGATGTGATGAGTGGTCAGTATTCGCAATAATTATGCGAGA GATGGGTGTCCGCCCCAAAGCCTGGTTACGACAATTGGGCACCCTAATCTTTGTACCATC TGATCCC

>Helianthus_annuus

GATCCTTCAGGCAAGTTTTGAAGGGAGATGAGGAGTGGTCAATGCTTGCATTTAAATATG CAAGATGGGGTGTCCGCCCAAAGCCTGTTACGACAATTGGGCACCTCCATTTTTAAACCA TCTGATCCC

$>$ Manihot_esculenta

GATCCTTCATGGCAGTTAACGGGATGTGATGAGTGGTCATTTTTCGCGATTAGCTTTGCG AGATGGGTGTCCGCCCCAATGCCAGTTACGACAATACGGGCACTCCCATTAATACCAATC TGATCCC

>Solanum_tuberosum

GATCCTTCAAGCAAGTTTTAATGGGATATGATGAGTGGTCATTACTCGCAATAAAATAAA ATATGCGAGAAGAGCGTTCCAGCCCAGCCTGGTTACGACAATTTGGGCGCTCTCATTTTT ACCAATCTGATCCC

$>$ Prunus_dulcis

GATCCTTCAGGCAGAAACGGGATGTGATGAGTGGTCAGTATTCGCAATAATTATGCGAGA GATGGGTGTCCGCCCCAAAGCCTGGTTACGACAATTGGGCACCCTAATCTTTGTACCATC TGATCCC

$>$ Solanum_lycopersicum

GATCCTTCAGGCAAGTTTTAATGGATGTGATGAGTGGTCATAACTCGCAATAAAAATGCG AGAAGAGCGTTCCAGCCCAGCCTGGTTACGACAATTTGGGCGCTCTTAATTTTACCAATC TGATCCT

>Theobroma_cacao_Belizian_Criollo_B97-61_B2

GATCCTTCAGGCAGTAAACGGGATATGATGAGTGGTCATTACTCACAAGCGCTGTGAGAT 
bioRxiv preprint doi: https://doi.org/10.1101/2021.11.30.470322; this version posted November 30, 2021. The copyright holder for this preprint (which was not certified by peer review) is the author/funder. All rights reserved. No reuse allowed without permission.

GGGTGTCCGCCTCAACGCCTGGTTACGACAACATGGGCACTCCCCTCTGTACCCCCTGAT

CCC

$>$ Capsicum_annuum

GATCCTTCAGGCAAGTTTTAATGGGATATGATGAGTGGTCATTACTCGCAATTTAAAAAT

ATGCGAGATGGGCGTCCAGGCTCAGCCTCGTTACGACATTTTGGGCGCTCTCATTTTTAA

CCAATCTGATCCC

$>$ Prunus_avium

GATCCTTCAGGCAGAAACGGGATGTGATGAGTGGTCAGTATTCGCAATAATTATGCGAGA

GATGGGTGTCCGCCCCAAAGCCTGGTTACGACAATTGGGCACCCTAATCTTTGTACCATC

TGATCCC

$>$ Nicotiana_attenuata

GATCCTTCAGGCAAGTTTAAAGGGGATATGATGAGTGGTCAATGCTCGCATTTAAATATG CGAGAAGGGCGTCCAGCTCAGCCTGTTACGACAATTTGGGCGCTCTCATTTTACCAATCT GATCCC

$>$ Gossypium_raimondii

GATCCTTCAGGCAGCAAACGGGATATGATGAGTGGTCATTACTCGCAAGCGTTGCGAGAT GGGTGTCCGCCGCAAAGCCTGGTTACGACACTTGGGCACTCCCATTTTTACCATCTGATC CC

$>$ Olea_europaea_var._sylvestris

GATCCTTCAGGCAAGTTTTACGGGGATGTGATGAGTGGTCGACACTCGCACTGAACATGC GAGATGGGTGTCCAGCCTAAGCCTGGTTACGACAATATGGGCACTCTCATTTTCACCAAT CTGATCCC

$>$ Malus_domestica

GATCCTTCAGGCAGAAACGGGATGTGATGAGTGGTCATAATTCGCAAATTAAAATGCGAG AGATGGGTGTCTGCCCCAAAGCCTGTTACGACAATTGGGCACTCTCCTGTTTTACCATCT GATCCC

$>$ Pistacia_vera

GATCCTTCAGGCGGTTTAACGGGGATGTGATGAGTGGTCATCACTCGCTATAACTTTGCG AGATGGGTGTCCGACCTATGCCTGGTTACGACAATCTGGGCACTCCCATCTTTACCAATC TGATCCC

>Populus_trichocarpa

GATCCTTCAGGGTTTAACGGGATATGATGAGTGGTCAATGCTCGCATATAAACATGCGAG ATGGGTGTCCAGCCCACTCCCTGGTTACGACAACAGGGCACTCCCATCTTCACCTATCTG ATCCC

$>$ Citrus_clementina

GATCCTTCAGGCAGTAACGGGGATGTGATGAGTGGTCATCGCTCGCTAAAAGTCTGCGAG ATGGGTGTCCGCCCCAAAGCCTGGTTACGACAATCTGGGCACTCCCATCTTTACCGATCT GATCCC

$>$ Coffea_canephora

GATCCTTCAGGCAGTTTACGGGGATGTGATGAGCGGTCAAAGCTCGCATTTAAACATGCG AGATGGGTGCCCGGCCCAAAGCCTGGTTACGACAACCCGGGCGCTCCCATATTTACCGAT CTGATCCC

$>$ Ipomoea_triloba

GATCCTTCAGGCAAGTTTTAAGgGGATGTGATGAGTGGTTAATGCTCGCAGTTTGTGCGA GAAGGGTGCCCGCCTTGCCTGTTACGACATATTGGGCATTCCAATGTTTTACCATCTGAT TCC

$>$ Vitis_vinifera

GATCCTTCAGGCAGTTAACGGGATATGATGAGTGGTCAACGTTCGCAACAAATATGCGAG ATGGGTGTCCGCCCTAGCCTGGTTACGACAATTGGGCACTCTCATCTTTACCAATCTGAT CCC

$>$ Trifolium_pratense

GATCCTTCAGGCAGAAACGGGGATATGATGAGTGGTCTGAGTCTCGCAATAAAAATGCGA GTTGGGTGTTCGCCCCAAGCCTGGTTACGACAATTGGGCACTCTCCTTTAATCAATCTGA TCCC

>Brachypodium_distachyon

GATCCTTCAGGCAGCAAAGGGAAATGATGAGCGGTAACGGCTTGCAAATTTCACTGCGAG AGGGTGCCCGCCCCAACTAAGCCTGTTACGACAATGGGGCACCCCAATGATTTACCATCT GATCCC

$>$ Eragrostis_tef

GATCCTTCAGGCAGTCAAAGGGATATGATGAGTGGTAAAAGCTCGCAAATTTATCTGCGA GAGGGGTGCCTGCCCCAAAATAAGCCTGTTACGACAACAGAGCACTCACATGCTTCACCA 
bioRxiv preprint doi: https://doi.org/10.1101/2021.11.30.470322; this version posted November 30, 2021. The copyright holder for this preprint (which was not certified by peer review) is the author/funder. All rights reserved. No reuse allowed without permission.

TCTGATCCC

$>$ Triticum_dicoccoides

GATCCTTCAGGCAGCAAAAGGGAGATGATGAGTGGTATCAGCTCGCTACAATGCGAGAGG GGTGTCCGCCCCAACCAAGCCTGTTACGACAATGGGGCGCCCCAGTGACTTACCATCTGA TCCC

>0ryza_nivara

GATCCTTCAGGCAGTTAAAGGGAGATGATGAGTGGTAACATCTCGCTAATTAATGTTGCG AGAGGGGTGTCTGCCCCAACCAAGCCTGTTACGACAACAGGGCGCTCCAATATTTTACCA TCTGATCCC

$>$ Oryza_glumipatula

GATCCTTCAGGCAGTTAAAGGGAGATGATGAGTGGTAACATCTCGCTAATTAATGTTGCG AGAGGGGTGTCTGCCCCAACCAAGCCTGTTACGACAACAGGGCACTCCGATATTTTACCA TCTGATCCC

>0ryza_glaberrima

GATCCTTCAGGCAGTTAAAGGGAGATGATGAGTGGTAACATCTCGCTAATTAATGTCGCG AGAGGGGTGTCTGCCCCAACCAAGCCTGTTACGACAACAGGGCACTCCAATATTTTACCA TCTGATCCC

>oryza_longistaminata

GATCCTTCAGGCAGTTAAAGGGAGATGATGAGTGGTAACATCTCGCTAATTAATGTTGCG AGAGGGGTGTCTGCCCCAACCAAGCCTGTTACGACAACAGGGCACTCCAATATTTTTACCA TCTGATCCC

$>$ oryza_barthii

GATCCTTCAGGCAGTTAAAGGGAGATGATGAGTGGTAACATCTCGCTAATTAATGTCGCG AGAGGGGTGTCTGCCCCAACCAAGCCTGTTACGACAACAGGGCACTCCAATATTTTACCA TCTGATCCC

$>$ Oryza_meridionalis

GATCCTTCAGGCAGTTAAAGGGAGATGATGAGTGGTAACATCTCGCTAATTAATGTTGCG AGAGGGGTGTCTGCCCCAACCAAGCCTGTTACGACAACAGGGCACTCCAATATTTTACCA TCTGATCCC

$>$ Oryza_brachyantha

GATCCTTCAGGCAGTTAAAGGGAGATGAGGAGTGGTAAAATCTCGCTATTTTCCGCTGCG AGAGGGGTGCCCGCCCCAACCAAGCCTGTTACGACAATTGGGGCATCCCCGTGTCTACCA TCTGATCCC

$>$ Oryza_punctata

GATCCTTCAGGCAGTTAAAGGGAGATGATGAGTGGTAACATCTCGCTAGTTATGTTGCGA GAGGGGTGTCTGCCCCAACTAAGCCTGTTACGACAACAGGGCACTCCAGTCATTTACCAT CTGATCCC

>Oryza_sativa_Indica_Group

GATCCTTCAGGCAGTTAAAGGGAGATGATGAGTGGTAACATCTCGCTAATTAATGTTGCG AGAGGGGTGTCTGCCCCAACCAAGCCTGTTACGACAACAGGGCACTCCAATATTTTACCA TCTGATCCC

>oryza_rufipogon

GATCCTTCAGGCAGTTAAAGGGAGATGAGGAGTGGTAACATCTCGCAACTTTTACTGCGA GAGGGGTGCCCGCCCCAACCCAAGCCTGTTACGACAACAGGGGCATCCCCATCATTTTAC CATCTGATCCC

$>$ Ananas_comosus

GATCCTTCAGGCAGTAAACGGGATGTGATGAGCGGTAACAGCTCGCATTCTGCGAGAGGG GCACCCGCCCCCATAAGCCTGTTACGACAAACAGGGGCGCTCTCGTAATTTACCAATCTG ATCCC

>Saccharum_spontaneum

GATCCTTCAGGCAGTTAAAGGGAAATGATGAGTGGTAAAAGCTCGCGTTTTTTCAATTGC

GAGAGGGGTGCCTGCCCCAAATAAGCCTGTTACGACAACAGGGCGCTCACACTGTTTACC ATCTGATCCC

>Punicum_hallii_FIL2_Phallii

GATCCTTCAGGCAGTCAAAGGGAAATGAGGAGTGGTATAAGCTCGCAATTTATTATGCGA GAGGGGTGCCTGCCCCAACTAAGCCTGTTACGACAACAGGGGCACCCCAATGAATTTACC ATCTGATCCC

$>$ Hordeum_vulgare

GATCCTTCAGGCAGCAAAGGGAGATGATGAGCGGTGTCAGCTCGCTATATGCGAGAGGGG TGTCCGCCCCAACTAAGCCTGTTACGACAATGGGGCACCCCAATGACTTACCATCTGATC CC 
bioRxiv preprint doi: https://doi.org/10.1101/2021.11.30.470322; this version posted November 30, 2021. The copyright holder for this preprint (which was not certified by peer review) is the author/funder. All rights reserved. No reuse allowed without permission.

$>$ Sorghum_bicolor

GATCCTTCAGGCAGTTAAAGGGAAATGATGAGTGGTAGAAGCTCGCGTTTTTTTATTGCG AGAGGGGTGCCTGCCCCAAATAAGCCTGTTACGACAACAGGGCACTCACACTGTTTACCA TCTGATCCC

$>$ Triticum_turgidum

GATCCTTCAGGCAGCAAAAGGGAGATGATGAGTGGTATCAGCTCGCTACAATGCGAGAGG GGTGTCCGCCCCAACCAAGCCTGTTACGACAATGGGGCGCCCCAGTGACTTACCATCTGA TCCC

$>$ Aegilops_tauschii

GATCCTTCAGGCAGCCAAAAGGGATGTGATGAGTGGTATCAGCTCGCTATAATGCGAGAG GGGTGTCCGCCCCAACTAAGCCTGTTACGACAATGGGGCGCCCCAGTGACTTACCATCTG ATCCC

$>$ Triticum_urartu

GATCCTTCAGGCAGCAAAAGGGATGTGATGAGTGGTATCAGCTCGCTATGTGCGAGAGGG GTGTCCGCCCCAACTAAGCCTGTTACGACAATGGGGCACCCCAGTGACTTACCATCTGAT CCC

$>$ Panicum_hallii_HAL2

GATCCTTCAGGCAGTCAAAGGGAAATGAGGAGTGGTATAAGCTCGCAATTTATTATGCGA GAGGGGTGCCTGCCCCAACTAAGCCTGTTACGACAACAGGGGCACCCCAATGAATTTACC ATCTGATCCC

$>$ Musa_acuminata

GATCCTTCAGGCAGTAAACGGGATGTGATGAGTGGTAAAAGCTCGCGCTGTTTGCGTGAA GAGCAGCCGCCCAGAGCCTGTTACGACAACAGGGCCTGCTCCCATCTTTACCCTTCTGAT CCC

$>$ Triticum_aestivum

GATCCTTCAGGCAGCAAAAGGGATGTGATGAGTGGTATCAGCTCGCTATAATGCGAGAGG GGTGTCCGCCCCAACTAAGCCTGTTACGACAATGGGGCGCCCCAGTGACTTACCATCTGA TCCC

$>$ Leersia_perrieri

GATCCTTCAGGCAGTTAAAGGGAGATGAGGAGTGGTAACAGCTCGCCATTTTTACTGCGA GAGGGGTGCCCGCCCCAACCAAGCCTGTTACGACAACAGGGCATTCCCTTCATCTTACCA TCTGATCCC

$>$ Zea_mais

GATCCTTCAGGCAGTTAAAGGGATATGATGAGTGGTAAAAACTCGGTTTTTTTATTCCGA GAGGGGTGCCTGCCCCAAATAAGCCTGTTACGACAACATGGCGCTCACATTTTTTACCAT CTGATCCC

>Eragrostis_curvula

GATCCTTCAGGCAGTCAAAGGGATATGATGAGTGGTAAAAGCTCGCAAATTTATCTGCGA GAGGGGTGCCTGCCCCAAAATAAGCCTGTTACGACAACAGAGCACTCACATGCTTCACCA TCTGATCCC

$>$ Setaria italica

GATCCTTCAGGCAGTTAAAGGGACATGATGAGTGGTAAAAACTCGCGATTTTACTGCGAG AGGGGTGCCTGCCCCAAATAAGCCTGTTACGACAACAGGGCACTCACAGCGTTTACCATC TGATCCC

$>$ Dioscorea_rotundata

GATCTTTCAGGCGGTCCAATGGGAGGTAATGAGTGGTAAAAGCTAGCGCTTTGATGCGTG ATGGGTGTGCAAAGCCTGTTACGACAATGAGCACACTCTAATCTTTGCCAATATGATCCC

- Fungi (budding yeasts) - See also Sharma et al (2017) PLos Genet

>S_cerevisiae

ATGACCTTCCAAGTTTTTAAAAGAATACGATGATATTATTTGCGTTTCAAATCGAACAAT TCTTCTCGGAGCGATCTGAGGTTTTAATGGAGATAGCGGTTCCTGCGCAACCCATTGATC TTGTTACATTCTTAAGAATGACAAGGACGCTTTTATAAAATTCTGATTCTTT

$>\mathrm{K}$ lactis

ATGACCTTCCAAGTTTTAAAAGAAGACGATGATACTATTTGCGCTTTTTTAATTCTTACA ATTCTTTCGTGGATGGGAATTATTTTCTGTTCATGGTTTTAATGGAGAATGTGGTGCCCT CGCAAGCCTTATAATCTTGTTACATTCTTTGAATGATTTGGGACACTTTTTATAAAATTC TGATTCTTT

$>$ C_Glabatra 
bioRxiv preprint doi: https://doi.org/10.1101/2021.11.30.470322; this version posted November 30, 2021. The copyright holder for this preprint (which was not certified by peer review) is the author/funder. All rights reserved. No reuse allowed without permission.

ATGACCTTCCAAGTTTATTAAGAAGACGATGAAATTATTTGCGTGAATCCTATTCGAACA ATTCTTCTTGCGATCCTATCGTGAGGTTTTAATGGAGAAAGCGGTACCAACGCAGACCTT ATTTCTTGTTACATCCCTAGTTTCTTTCGAGGAGCTAGTTTTCTGGACGCTACTAGAAAA TTCTGATTCTT

>S_eubayanus

ATGACCTTCCAAGTTTTTAAAAGAACACGATGATACTATTTGCGTTTCAAATCGAACAAT TCTTCTCGGAGCAATCTGAGGTTTTAATGGAGATAGCGGTTCCTGCGCAGCCAATTGATC TTGTTACATTCTTAAGAATGACAAGGACGCTTTTATAAAATTCTGATTCTTT

$>$ C_albicans

ATGACCTTCCAAGTTTTATACTTACATGATGAAATCTCGCGTTATATTCAACGATTCGGC CCTTCTGTTGACTGGTTTTCTTTTATGAGAAGTTGAGTCTTTTTCGGAGGATTCCTTTTT GTAAGTTCATGGGGAAACTGGTTAGTAGAAAATGACTTGACCGTCAGGGTAATGAAGTTG ATGAAGGTGTCTGGCGCAACCATTATCTTGTTACATATATTTCAGACGCTATAGAGAACT TCTGACCTA

$>$ A_gossypii

ATGACCTTCCAAGTTTTATAAAGAAGTGGATGATATGATTTGCGTAGCAACTCGAACAAT TCCTCGCCTGTGATCAGGCTGAGGTTTTAATGGAGAAAGTGGTGCCCGCGCAGCAAATAA TCTTGTTACATTCTTTGAATGCCTGGGACACTTGCTATAAAATTCTGATTCTT $>$ L_fermentati

ATGACCTTCCAAGTTTTATAAAGAAGAAGATGAAATTATTTGCGTTTCAATTCGAACAAT TCTTCTCAGTGTAGCTTAGCTGTACTTGAGGTTTTGATGGAGAATGTGGTTCCTGCGCAG CTTTTTAATCTTGTTACATTCAATGAATGCTCAAGGACACTTTTTAGAAAATTCTGATTC TTT

$>$ S_passalidarum

ATGACCTTCCAAGTTTTATACTTACATGATGAAATCTCGCGTTTTAAATCATTCGACAAT TCCCTTTCGAGGTTGAGTTGTTCCCTTCGGGAACGATTCCTTTGGAAAATGCTTCTTTCC AGGAGCTGGGTTTTAATGGTGACGGTGTCCGGCGCAACAATTCTTGTTACTAAATTTACG GACGCTGAGGAGAGCTTCTGACCCC

$>C \_l u s i t a n i a e$

ATGACCTTCCAAGTTTTATAACAAATGATGAAGTCTCGCGTGCATCTATACTATTCCCTC TGGAGTTGCAGTCGGAGCTTTCGGGCTCTGACTCCTCCGGAAATGCTTTTCCGTACGGGA AAGTTGGGTTTGTGTGGTGAAAGTGTTTGGCGCAACCTTTCTTGTTACATTTGACAAACA CTGAAAGAATTTCTGATCCAA

$>C$ jadinii

ATGAACCTTCCATAGTTTTAAACTAACGTGATGAAATCTCGCGTCTATCTCTTCTAACGA TTCTTCCCTCGGAGGTGTGAAAGCACATTTCGAGAATGGATGCCTTTTAGGCGTTCTGGG TTTCGATGGGGATGGGCATTCGACGCAACCTTTTCTTGTTACATAACTATTTGGATGCTC TAAGGAGAAGTTCTGATTTAC

$>$ A_rubescens

ATGACCTTCCAAGTTTTATAACTATGATGAAACTTAGCACTTCTGAAACTCTGACCGATT CCCTTTCCTATGGAAATTGCCGGTGCTCAGCGCCGGCTTGGGTTTTGAATGGAGTCAACG CTTGGTGCACAATTCTTGTTACAACTTAAAGCGTTTGCTATTTCTGATCCTT

$>$ W_anomalus

ATATCCTTCCAAGTTTTATACTATATGATGAAGTCTCGCGTTTTCTTCTAACAATTCCCC AAGGTTGAGTGACTGGCAACGGTTATTCCTTTGAATGGTGTGGTTTAAATCATATCTGGG TTTTAATGGAGATGGTGTTCGGCGCAAAATTATCTTGTTACAATTTAATTATTAGGACGC CTAAATGAGAAATTCTGACCCCT

>S_stipitis

ATGACCTTCCAAGTTTTATACTCATATGATGAAATCTCGCGTTTCTATCCATGACAGATC CCTCTGGAGTTGAGTTGAAGTTCTGCTTCGATTCCTCCGGAAACGGCCTCTTGTACGAGA GACCTGGGTTCTACTGGTGAGAGCGTTTGGCGCAACCATTCTTGTTACATAAAGAAAAGA CGCTGAAAGAACTTCTGACCCC

$>$ D_hansenii

ATGACCTTCCACGTTTTATACTCATGATGAAATCTCGCGTTATTCAAGACAATTCCCTTT AGAGTTGTAGTCTTTCTTTCGGGAGAGATTCCTCTGAAATTGCTTTCTCATACGAGAGAG TAGGGTTTTATTGGTTAAGGTGTTTGGCGCAAACTATTCTTGTTACAAAAATATCACAGA CGCTTAAGAGAACTTCTGAACCTC

$>B$ inositovora

ATGAACCTTCCAAGTTTTATACTCATATGATGAAGTCTCGCGTTTCCTCATGACAGCTGG CCCCTCTCCTGAAGTTGAGTTACCTCACGGTGATTCCTTTGGGGGGGAATTTAGTCCTTG TATGAGGGCTAGGGTGCTAATGGTGATGGTATTCGGCGCACAACCATAATCTTGTTACAA 
bioRxiv preprint doi: https://doi.org/10.1101/2021.11.30.470322; this version posted November 30, 2021. The copyright holder for this preprint (which was not certified by peer review) is the author/funder. All rights reserved. No reuse allowed without permission.

TTTTATTTAACGGATGCTTTGGAGAATTTCTGACCCC

$>$ P_tannophilus

ATGACCTTCCAAGTTTTATTTACATGATGAAATCTCGCGTACTCCATATTATGACTTTTC CCTTCTTCTTTTTTTTATTCCGTATTTCAAGCTGGTTTTGTTTCGCCTTCACAGGCTTGA GACAAGATTAGATTTGGAAGGAATTTCTATATGAAGAAAAATTTTCTTTTCCTTATGGGA AGGAAAATGGGTTTGAGTGGGTAATGGCGATTGGCGCAAAACTTTTCTTGTTACACAATT AATTTTTACAGTCGCTATAAATGAGATGTTCTGACCCTT

$>0 \_$polymorpha

ATTATCCTTCCTAGTTTTTACTAAGAAATGATGAAAACTAGgCGCTGTTTTTTAACACAC CCGGTTTCCCTCCTTCGGGAATAAGTTGTTCTTCGGGATGGCTTTGGGAAAGCGTGGGTG AAAGCGTTTGGCGCAAATTTATCTTGTTACAACATTTGTTGCAAGACGCTTTTGTACCTT CTGAATT

$>$ P_membranifaciens

ATGACCTTCCTAGTTTTTTACTATACATGATGAAAACTAGGTGTTGTCTGTTGACACATC CGGCATCCCTCTTCGGAAGTGCTGTCTTTATGGCAGCCGGGATGGTATGGGTGACTGCGT TTGGCACAAATTTCTTGTTACATAAAACGACGCTTTCATTTTGTACATTCTGACCCC

$>$ L_elongisporus

ATGACCTTCCAAGTTTTATACTACATGATGAAATCTCGCGTTTATTCATTCATTGATTAA AGTCCCTTTCTCAAAGTTGTAGTCGGGGTCTTCTTTCGTTTGAGAGGGGGCTCTTGATTC CTTTGAGAGATGTGTCTCTTGACGGAGGCTGGGTATTTAAATGATGAAAGGTGTCTGGCG CAACCTTATCTTGTTACATTTTACAGACGCTTAAAGAGAACTTCTGACCCT

$>$ T_phaffii

ATGACCTTCCAAGTTTTTAAAAGGATATGATGAAGTTATTTGCATTTTTAATCGAACAAT TCTTCTCAATATAATTGAGGTTTTAATGGAGATTGCGGTGCCTGTGCAGAAAATTATCTT GTTACATTCAATTGAATGAATCAGGACGCTATATTAGAAAATTCTGATCCTT

$>$ Y_lypolytica

ATGACCTTCCACGTTTTAAACGTTTATGATGAAAATTATGCGTGCTAGCTGATTTGCCCC CTCCTCGGAGGTAGTCCTTGACCGGACGGCGGCATCTTATGTTGATAGCGTTTGGCGCAC TTACCTTGTTTAAACAAACGCGGTAGTACTTTCTGATCCTT

$>N_{-}$fulvescens

ATGACCTTCCACGTTTTATAATGTGTCATGATGAAAACTACGCGTTTATCAACTTAGCTT GCCCCCTGGTCGAGTCTACTACGTTGATAATTTGGAGGAAAGGAACTTGTTTTTTTTCAC TAGGTTATTGACCGGTGGAATCCTGGGCGTCCCTGACCGGACTGGGCGTAGCATGGTGAT AGCGTTCGGGCGCAACATTACCTTGTTTTTACGAACGCTTTTGTACTTTCTGATCCCT

$>$ L_starkeyi

ATGACCTTCCAGCGTTTTACGTTGTACATGATGAAAACCAGGCGTTAGCTGTCGGATATG TGTCCGGGTGATTTAGTTCGCCTTTGCGACCGTATGTATGTGATGACGGCGTTCGACGCA ACCTCTTGTTACGTTACCAGGACGCTTTTGGTACCTTCTGATTTTC

$>$ E_cymbalariae

ATGACCTTCCAAGTTCTATAAAAAGGTGATGATATTATTTGCATTTCTAAATCGAACAAT TCTTCTCGTGAGCTTTATGCACCTCGAGGTTTTAATGGAGAATGTGGTGCCCGTGCAGCC TTATAATCTTGTTACATTCATTGAATGCCAAGGGACACTATAATCGTTAGATAATTCTGA TTTTC

$>$ V_polyspora

ATGAACCTTCCAAGTTTTAAAAAGAGTGCGATGATACTATTTGCATTTATTAAATCGAAC AATTCTTCTCGGTGGACTAGATAATATCTAATTTACTGAGGTTTTAATGGAGAATGCGGT TCCTATGCAGCCAATATAATCTTGTTACATTCAATTGAATGACAGGACGCTAATATTATG AAATTCTGATTCTT

$>$ T_blattae

ATGACCTTCCAAGTTTTATTAAGAATACGATGATATTATTTGCGTAATAAGCTAACAATT CTTCTCGTGGGGTTTCGGCTCTGTGAGGTTTTAATGGGGAATGCGGTTCCCGCGCAGCCA ACCAATCTTGTTACATTTTTTTTAAAATGATTTTTTGGGACGCTACAATATAAAATTCTG ATTCTTT $>L_{-}$thermotolerans ATGACCTTCCAAGTTTTATAAAGAAGTGGATGATATGATTTGCGTAGCAACTCGAACAAT TCCTCGCCTGTGATCAGGCTGAGGTTTTAATGGAGAAAGTGGTGCCCGCGCAGCAAATAA TCTTGTTACATTCTTTGAATGCCTGGGACACTTGCTATAAAATTCTGATTCTTT $>$ T_delbrueckii ATGACCTTCCAAGTTTTATAAAGAAGTGGATGATATGATTTGCGTAGCAACTCGAACAAT TCCTCGCCTGTGATCAGGCTGAGGTTTTAATGGAGAAAGTGGTGCCCGCGCAGCAAATAA TCTTGTTACATTCTTTGAATGCCTGGGACACTTGCTATAAAATTCTGATTCTTT 
bioRxiv preprint doi: https://doi.org/10.1101/2021.11.30.470322; this version posted November 30, 2021. The copyright holder for this preprint (which was not certified by peer review) is the author/funder. All rights reserved. No reuse allowed without permission.

$>$ Z_rouxii

ATGACCTTCCAAGTTTTAAAAGAAGAAGATGAAATTATTTGCGTCTATTAAATCGTACAA

TTCTTCCCTCGGGTTTTAAAGCTCGTGGGGTTTTAATGGTGAAGACGGTTCCCGCGCAGC

AATTTTTCTTGTTACACCAATTTTAATGGTTTTATAGGGACGTCTCTAGGAAATTCTGAT

TCTTT 
bioRxiv preprint doi: https://doi.org/10.1101/2021.11.30.470322; this version posted November 30, 2021. The copyright holder for this preprint (which was not certified by peer review) is the author/funder. All rights reserved. No reuse allowed without permission.

\section{Supplementary data $\mathbf{S 2}$}

>H_sapiens

MRQLQKKIKNGTLNIKQDDPFELFIAATNIRYCYYNETHKILGNTFGMCVLQDFEALTPN LLARTVETVEGGGLVVILLRTMNSLKQLYTVTMDVHSRYRTEAHQDVVGRFNERFILSLA SCKKCLVIDDQLNILPISSHVATMEALPPQTPDESLGPSDLELRELKESLQDTQPVGVLV DCCKTLDQAKAVLKFIEGISEKTLRSTVALTAARGRGKSAALGLAIAGAVAFGYSNIFVT SPSPDNLHTLFEFVFKGFDALQYQEHLDYEIIQSLNPEFNKAVIRVNVFREHRQTIQYIH PADAVKLGQAELVVIDEAAAIPLPLVKSLLGPYLVFMASTINGYEGTGRSLSLKLIQQLR QQSAQSQVSTTAENKTTTTARLASARTLYEVSLQESIRYAPGDAVEKWLNDLLCLDCLNI TRIVSGCPLPEACELYYVNRDTLFCYHKASEVFLQRLMALYVASHYKNSPNDLQMLSDAP AHHLFCLLPPVPPTQNALPEVLAVIQVCLEGEISRQSILNSLSRGKKASGDLIPWTVSEQ FQDPDFGGLSGGRVVRIAVHPDYQGMGYGSRALQLLQMYYEGRFPCLEEKVLETPQEIHT VSSEAVSLLEEVITPRKDLPPLLLKLNERPAERLDYLGVSYGLTPRLLKFWKRAGFVPVY LRQTPNDLTGEHSCIMLKTLTDEDEADQGGWLAAFWKDFRRRFLALLSYQFSTFSPSLAL NIIQNRNMGKPAQPALSREELEALFLPYDLKRLEMYSRNMVDYHLIMDMIPAISRIYFLN QLGDLALSAAQSALLLGIGLQHKSVDQLEKEIELPSGQLMGLFNRI IRKVVKLFNEVQEK AIEEQMVAAKDVVMEPTMKTLSDDLDEAAKEFQEKHKKEVGKLKSMDLSEYIIRGDDEEW NEVLNKAGPNASIISLKSDKKRKLEAKQEPKQSKKLKNRETKNKKDMKLKRKK

$>M$ musculus

MNRKKVDNRIRILIENGVAERQRSLFVVVGDRGKDQVVILHHMLSKATVKARPSVLWCYK KELGFSSHRKKRMRFSHNKIMSGTLYLKQDDPFELFVAATNIRYCYYNETHKILGNTFGM CVLQDFEALTPNLLARTVETVEGGGLVVILLRTMNSLKQLYTMTMDVHSRYRTEAHQDVV GRFNERFILSLASCKKCLVIDDQLDILPISSHVASIEALPPQAPDENLSPAALELLELKE SLQDTQPVGVLVDCCKTLDQAKAVLKFIEGISEKTLRSTVALTAARGRGKSAALGLAIAG AVAFGYSNIFVTSPSPDNLHTLFEFVFKGFDALQYQEHLDYEIVQSLNPEFNKAVIRVNV FREHRQTIQYIHPADAVKLGQAELVVIDEAAAIPLPLVKSLLGPYLVFMASTINGYEGTG RSLSLKLIQQLRQQSAQSQVSTTAENKTTTTARLASARTLHEVSLQESIRYAPGDAVEKW LNDLLCLDCLNITRIVSGCPLPEACELYYVNRDTLFCYHKASEVFLQRLMALYVASHYKN SPNDLQMLSDAPAHHLFCLLPPVPPTQNALPEVLAVVQVCLEGEISRQSILNSLSRGKKA SGDLIPWTVSEQFQDPDFGGLSGGRVVRIAVHPDYQGMGYGSRALQLLQMYYEGKFPCLE EKVLETPQEIRTVSSEAVSLLEEVITPRKDLPPLLLKLNERPAERLDYLGVSYGLTPRLL KFWKRAGFVPVYLRQTPNDLTGEHSCIMLKTLADEDEAEQGAWLAAFWKDFRRRFLALLS YQFSTFSPALSLNIIQNRNVAKSALPALGREHLEALFLPYDLKRLEMYSRNMVDYHLIMD LIPAISRLYFLNQLGDLSLSAAQSALLLGIGLQHKSVDQLEKEIELPSGQLMGLFNRIIR KVVKLFNDVQEKAIEEQMVAVKDVVMEPTMKTLSDDLDEAAKEFQEKHKKEVGKLKDMDL SQYVIRGDDEEWNEVLSKAGQNASIVSLKSDKKRKLETKQEPKQS

$>$ R_novergicus

MHRKKVDNRIRILIENGVSERQRSLFVVVGDRGKDQVVILHHMLSKATVKARPSVLWCYK KELGFSSHRKKRMRQLQKKIKSGTLNIKQDDPFELFVAATNIRYCYYNETHKILGNTFGM CVLQDFEALTPNLLARTVETVEGGGLVVILLRTMNSLKQLYTMTMDVHSRYRTEAHQDVV GRFNERFILSLASCKKCLVIDDQLNILPISSHVASIEALPPQAPDENLSPAALELQELKE SLQDTQPVGVLVDCCKTLDQAKAVLKFIEGISEKTLRSTVALTAARGRGKSAALGLAIAG AVAFGYSNIFVTSPSPDNLHTLFEFVFKGFDALQYQEHLDYEIVQSLNPEFNKAVIRVNV FREHRQTIQYIHPADAVKLGQAELVVIDEAAAIPLPLVKSLLGPYLVFMASTINGYEGTG RSLSLKLIQQLRQQSAQSQVSTTAENKTTTTARLASARTLHEVSLQESIRYAPGDAVEKW LNDLLCLDCLNITRIVSGCPLPEACELYYVNRDTLFCYHKASEVFLQRLMALYVASHYKN SPNDLQMLSDAPAHHLFCLLPPVPPTQNALPEVLAVVQVCLEGELSRQSILNSLSRGKKA SGDLLPWTVSEQFQDPDFGGLSGGRVVRIAVHPDYQGMGYGSRALQLLQMYYEGRFPCLE EKVLETPQEIHTVSSEAVSLLEEVITPRKDLPPLLLKLNERPAERLDYLGVSYGLTPRLL KFWKRAGFVPVYLRQTPNDLTGEHSCIMLKTLADEDEAERGAWLAAFWTDFRRRFLALLS YQFSTFSPALSLNIIQNRNIAKSAPPVLGREHLEALFLPYDLKRLEMYSRNMVDYHLIMD LIPAISRLYFLNQLGDLALSAAQSALLLGMGLQHKSVDQLEKEIELPSGQLMGLFNRIIR KVVKLFNDVQEKAIEEQMVAVKDVVMEPTIKTLSDDLDEAAKEFQEKHKKDVGKLKDMDL SQYIIRGDDEEWNEVLSKAGQNASIVSLKSDKKRKLETKQEPKQSKKLKKSGNNRKDTKL KRKK

$>$ G_gallus

MQRRKVDNRIRVLIENGVAERQRGLFVVVGDRGKDQVVILHHMLSKATVKARPSVLWCYK KELGFSSHRKKRMRQLQKKIKSGTLNIKDDDPFELFIAATNIRYCYYNETHKILGNTFGM CVLQDFEALTPNLLARTVETVEGGGIVVILLRTMNSLKQLYTMTMDVHSRYRTEAHQDVV 
bioRxiv preprint doi: https://doi.org/10.1101/2021.11.30.470322; this version posted November 30, 2021. The copyright holder for this preprint (which was not certified by peer review) is the author/funder. All rights reserved. No reuse allowed without permission.

GRFNERFILSLASCKNCIVIDDQLNILPISSHVANITPVPPQSQEDSLRPQDLELKELKE

SLQDTQPVGVLVDGCKTLDQAKAVLKFIEAISEKTLRSTVALTAARGRGKSAALGLAIVG AVAFGYSNIFVTSPSPDNLHTLFEFIFKGFDALQYQEHLDYEIIQSLNPEFNKAVVRVNV FKEHRQTIQYIHPADSVKLGQAELVVIDEAAAIPLPLVKKLLGPYLVFMASTINGYEGTG RSLSLKLIQQLRQQSAQAQVTMTAENKSTATAKLASARTLHEVSLHESIRYAPGDPVEKW LNDLLCLDCLSITRI ISGCPLPEDCDLYYVNRDTLFCYHRASELFLQRLMALYVASHYKN SPNDLQMLSDAPAHHLFCLLPPVPPTQNSLPEVLAVVQVCMEGEISRQSIMNSLSRGKKA SGDLIPWTISEQFQDPDFGALSGGRVVRIAVHPDYQGMGYGSRALNLLQMYYEGKFPCLE EKTVQKPKEIATVSSETVSLLEEVVTPRKDLPPLLLKLSERQAESLDYLGVSYGLTPRLL KFWKRAGFVPVYLRQTPNDLTGEHSCIMMKMLNEEDVEQEPWLTAFWKDFRRRFLSLLSY QFSTFSPSLALNILQNKNIKQQSQPPISRAELESAFIPYDLKRLEMYSRNMVDYHLIMDM VPTIARMFFLNQLGDISLSAAQSALLLGIGLQHKSLDQLEKEVELPSSQLMGLFNRIIRK VVQLFNTVQEKAVEEQMVATKDIVMEPTIKSLNDDLEEAAKEFQEKHKQEVGKLKEMDLT QYIIRGDDEEWNEVLSKAGQNASI ISVKSEKKRKLETVRGHKQQKKFKKTKDIKQKRKK $>$ D_rerio

MATFRKKVDNRIRVQIENGVAEHHRSMFVIVGDRGRDQVVILHHMLSKATVRARPSVLWC YKKELGFSSNRKKRMRQLQKKIKTGTLNLNQDDPFELFVAATNIRYCYYNETHKILGNTF GMCVLQDFEALTPNLLARTVETVEGGGIVVILLRTMNSLKQLYTMTMDVHSRYRTEAHQD VIGRFNERFILSLSSCKSCVVIDDQLNILPISSHATNIKPIPPKKQDDGLSPREQELKDL KESLQDTQPVGVLVDGCKTLDQAKAVLKFIEAISEKTLRSTVALTAARGRGKSAALGLAM AGAVAFGYSNIFVTSPSPDNLHTLFEFVFKGFDALQYQEHLDYEIIQSLNPEFNKAVVRV NIFKEHRQTIQYIHPADAVKLGQAELLVIDEAAAIPLPLVKKLLGPYLVFMASTINGYEG TGRSLSLKLIQQLRQQSSESQQNLSAENRNTSTARLAAARSLHEVTLHESIRYGQGDHVE RWLNDLLCLDCLSVPRI ISGCPLPQTCDLYYVNRDTLFCYHKASEAFLQRLMALYVASHY KNSPNDLQMLSDAPAHHLFCLLPPVPPTQNSLPEVLAVLQVCLEGEISRQSILSSLSRGK KASGDLIPWTVSEQFQDSEFAGLSGGRIVRIAVNPDYQGMGYGTRALQLLQRYYEGQFPL WDEQEQTMTSAITSVSSEAVSLLEEVVSPRKDLPPLLLKLNERRAERLDYLGVSYGLTPQ PLKFWKKAGFVPVYLRQTPNDLTGEHSCIMLKDLNSEDLTDQNQWLSAFWKDFRRRFLSL MSFQFSKFSSTLALNILQNKSAKDDSTAVLTSAELAATFTPYDLKRLEMYSRNMVDYHLI MDMIPVIARMHFLKQFGDISLSVAQSALLLGIGLQHKSVDELEKEIGLPSSQLMGLFNRI IRKVVQFFNTLQERAVEAEMVATKDIGLEPTVQTLNEDLNDAAKEFDEKHKKDIEKIKDM NLSEYMIRGDDEEWDQVLKKAGHTAIVSIKGDKKRKLEGTNQKEQREGKKMKKIKDKRGK FGKKT

$>X \_l a e v i s$

MQRKKIDNRIRVLIENGVAQKHRSFFVVVGDHGKDQVVILHHMLSKATVRARPSVLWCYK KELGFSSHRKKKMRKLQKRIKSGSLNIKEDDPFELFVAATNIRYCYYNETHKILGNTFGM CVLQDFEALTPNLLARTVETVEGGGIVVLLLRTMSSLKQLYTMTMDVHSRYRTEAHQDVV VRFNERFILSLASCKTCMVIDDQLNILPISSHIANIKPIPPKTQDDSLSPEEQELQDVKL SLQDTQPVGVLVDNCKTLDQAKAVLKFIEAVSEKTLRSTVTLTAARGRGKSAALGLAIAG AVAFGYSNIFITSPSPDNLHTLFEFIFKGFDALQYQEHMDYEIIQSLNPEFEKAVVRVNV FKEHRQTIQYIHPADAVKLGQAELVVIDEAAAIPLPLVKKLMGPYLVFMASTINGYEGTG RSLSLKLIQQLRQQSADSQVTLTAENKSTASNKLVSARTLHEVTLQESIRYAPGDHVEKW LNDMLCLDCMNISRIISGCPLPETCELYYINRDTLFCYHKASESFLQRLVALYVASHYKN SPNDLQMMSDAPAHHLFCLLPPVPPTQNSLPEVLAVVQVCLEGEISRQSILSSLSRGKKA SGDLIPWTVSEQFQDPDFGSLSGGRVVRIAVHPDYQGMGYGSRALRLVQMYYEGQFPCLE ENAANKPQEITSISGEAVSLLEESLTPRKNLPPLLLRLSERPAEKLDYLGVSYGLTPKLL KFWKRAGFVPVYLRQTPNDLTGEHSCVMLKTLHEDEESDQEPWLTAFWKDFQKRFLSLLS YQFSTFTPALALNILQNRNIKKQSENLISRSQLEGALTPYDLKRLEMYSRNMVDYHLIMD LIPSLARMYFLQQLGGLSLSAAQSALLLGIGLQHKGVDQLEKEIELPSSQLMGLFNRIIR KVVQLFNRIQERAVEEEMVTTKEVVMEPTLKSLQEDLEEAGKEFQEKHKQEVEKLKGINL SEYVIRGDDEEWNEVLKKTGQNASVISMKSDMKRKLEKPEKEGFKQKKLKKNKEKKQKFA $>P$ marinus

MSRKKVDNRIRVLIENGFSQKYRTLFVVVGDKGKDQVVILHHMLSKAAVKARPSVLWCYK KELGFSSHRKKRMRQLQKKLKTGTLDVKHDDPFELFVAATNIRYCYYNETHKILGNTFGM CVLQDFEAMTPNLLARTIETVEGGGLAVILLRTMNSLKQLYTMTMDVHTRFRTESHQDVV GRFNERFILSLASCKTCMVIDDQLNILPLSTHTLCISPLPPKPQDESLSPSEIELGDLKE SLQDTQPVGVLVSCCKTLDQAKAVLKFIEAISEKTLRSTVALTAARGRGKSAAMGLAIAS AVAFGYSNIFVTSPSPDNLHTLFEFLFKGFDALQYQEHLDYEIIQSTNPEFNKAIVRVNI YKEHRQTIQYIHPGDAAKLGQAELVVIDEAAAIPLPLVKSLLGPYLVFMASTINGYEGTG RSLSLKLIQQLRQQSSQSQVTMKAENKNTAVSKLASARSLHEVTLDESIRYAPGDPVEKW LNDLLCLDCLNIQRVSVGCPVPDTCDLYYVNRDTLFCYHKASEAFLHRLMSLYVASHYKN 
bioRxiv preprint doi: https://doi.org/10.1101/2021.11.30.470322; this version posted November 30, 2021. The copyright holder for this preprint (which was not certified by peer review) is the author/funder. All rights reserved. No reuse allowed without permission.

SPNDLQMLSDAPAHHLFCLLPPVQATQNALPEVLAVIQVCLEGEISQQSIMSSLARGKRA

AGDLIPWTISEQFQDPDFGSLSGGRIVRIAVHPDYQGMGYGSRALRLLQMYYEGKFPCLD ETASQTQGHISSIASDAVGLLEETLTPRRDLPPLLHKLSERPTERLDYLGVSFGLTPQLL KFWKRSGFTPVYLRQTPNDLTGEHSCIMLKVLNEDEQMERPENWLAAYWKDFRRRFVTLL SYQFSGFLPAVSLNILQNRNVKEKPAQALSKSELEATFSPYDLKRLEMYSRNMVDYHLIM DLVPGIARLYFTHRLPDMSLSAAQLALLLGVGLQHKAVEQLEKEIDLPVSQLMGLFNRIM RKAVQVFTAIQETAIEEQMTKVKEVVMEPTVKSLAEDLEDAAKEFAEKHKKDLVQIKDMN LTEYVIRGDDKEWNEALKKVGPSASVVSITGEKRKSAEKDSSHDHKSKKSFQKGKPGKLG KPGKPGKPGGPGKAGKSRGGFKKQK

$>C$ _intestinalis

MRKKVDNRIRVLVENGVATKHRTMFFIVGDKCRNQVVILHHMLSKAQVRARPSVLWCYKK ELGFSTHRKKKMKQIQKRISSGTLDLKENDPFELFVAATNVRYCYYNETHKILGSTYGMC VLQDFEALTPNLLARTIETVEGGGIICFLLRSMTSLKQLYTMTMDVHARYRTESHSDVVG RFNERFLLSVASCNNCLIIDDELNVLPVSTNSQNIEAVPPKPAEDSLTPSEKELVDLKES LQDTQPVGCVINCCKTIDQGKALLKFVDAISEKTLRSTVVMTAARGRGKSATLGLAISAA VAFGYSNIFVTSPSPENLKTLFEFVFKGFDALEYQEHLDYELIQSTNPDFNKAIVRVNVF REHRQTIQYIHPSDSAKLSQAELLVIDEAAAIPLPLVQSLLGPYLVFMSSTINGYEGTGR SLSLKLVKQLRIQSATRNTATTKQTTASPLGRNLSEITLEESVRYAPTDPIESWLNELLC LDCGSSSFSKISTGCPPPSACDLYYVNRDTLFCYHKASEAFLQRLMSLYVASHYKNTPND LQMLSDAPAHHIFCLLPPVSAEQKSLPDVLCVLQICLEGEISKSSVVSSLSRGKRAAGDL IPWTVTEQFQDHDFASLSGARVVRIATHPDYQGMGYGSRALELLEKYYEGKMTSLDENET PAEEVNIVNEEEVEAMDEVLTPRKSLPPLLWKLSERRPEQLNYIGVSYGLTAPLLKFWSK AGYTSVYLRQTANDLTGEHSCIMLKALDTEVDKSWQDAYFDDFRRRFISLLHFQFSSFKS STALQVLRRKSKKTTKSNPLTKSELDFFLTSYDTKRLTLYSRNMADYHLVMDLLPTLARL VFLEKLVVPLSVAQKAI ILAIGLQHKSVETVASEIDLPSSQVLGLFNRAVRKITTHLSEI ESAAAEKELKSTLGDENAADAVNMQPLEESLDSELNAAAYEVVQKQKEELKQTLASMDLK QYEIGGSDEAWQDALSSGKKQNIVSVKSAKRPLPTSEEPDTKDKKKKRKFKKKVKSA $>$ B_floridae

MVLKKVDNRIRVLIENGVVQHHRGLFVIVGDKGKDQVVILHHMLSKAQVKARPSVLWCYK KELGFSTHRKKRMKQLQKKIRNGTLDVKEDDPFELFIAATNVRYCYYAETHKILGNTYGM CVLQDFEALTPNLLCRTIETVEGGGIACLLLRSMNSLRQLYTMAMDVHSRYRTEAHRDVV GRFNERFLLSLSSCETCMVIDDQLNILPISSHILNISAVPSRSKEDSLNPAEVELKGLKE SLQDTQPVGSLVNCCKTLDQAKALLKFIEAISEKTLRSTVAMTAARGRGKSAALGLAIAG AVAFSYSNIFVTSPSPENLNTLFEFVFKGFDALDYQEHMDYELVQSTNPDFHKAVVRVNI YREHRQTIQYLHPSDAQKLGQAELVVIDEAAAIPLPLVKSLMGPYLVFMASTVNGYEGTG RSLSLKLIQQLRQQNATFGGNSEAKAAAAMATKTEGGASATGRTLHELVLTESIRYASED PVEAWLNRLLCLDVSSVPRVHSGCPIPASCDLYYVNRDTLFCYHKASEAFLQRIMSIYVS AHYKNTPNDLQMLSDAPAHHLFVLLPPVDPDQSALPEVLCVVQVCLEGEISKQSIMSGLS RGKRASGDLIPWTVSQQFQDTEFASLSGGRVVRIATHPDYQGMGYGMRALQQLDMYYQGK VPSLSEDTGTAQGLPAVDKETVSLLEEKLGPRKNLPPLLSKLSERPAERLDYLGVSYGLT APLLKFWKKAGYAPVYLRQTPNDLTGEHSCIMLKRIQEDDEGQDTDWLSAFWTDFRRRFL SLLSYQFRTFQPALSLSILQAKKDVKTDTSVLTQGDLYAHLTQYDLKRLDLYSRNMVDYH LIMDLLPALARMYFLQKLDTSLSVAQSAILLGLGLQHKTVDDLERELELPASQVMGLFNR LVRKIVQFLSSIEEQAVESELLAPKEVVMEPTAQSLKTELAEAAKEFEDKQKKELKKLKD MDLEKYVIKGSETEWESALTSGLKSGVVSVASGKKRKEEAAPTPESTKKKSKKFKKDKKF KKT

$>$ S_purpuratus

MVRKKIDNRIRVQIENGVTQGHRSLFVLVGDKGKDQVVILHHMLSKAVVKARPSVLWCYK KELGFSSHRKKRMKQLQKKIKGGMVDVSDDDPFELFIAATNIRYCYYSETHKILGNTYGM CVLQDFEAMTPNLLARTIETVEGGGIVVILLRSVSSLQQLYTMSMDVHSRYRTESHQDVV ARFNERFLLSLASCDSCMVVDDQLQILPLSQHTLNIEAIPPRLRXVEDSQSPAEIELKEL KASLQDTQPVGALINCTKTLDQAKALLKFVESISEKTLRSTVSMTAARGRGKSAALGLAI ATAVAFGYSNIFVTAPSPENIRTLFEFIFKGFDGLDYQEHLDYELVQSTNPEFNKAVIRV NIFREHRQTIQYIHPGDAHKLSQAELVVIDEAAAIPLPLVKELLGPYLVFMSSTINGYEG TGRSLSLKLLQQLRKQNATFGVSSSAANKASTSLSTGSSASGRVLHEVTLEESIRYSEGD SVEKWLNELLCLNVAEVQRISSGCPLPADCDLYYINRDTLFSYHKASEVFLQRLMSIYVA AHYKNTPNDLQLLSDAPAHHIFCLLGPVKASQSGLPEILCVLQVCLEGEISKETIMSSLS RGKRASGDLIPWTISQQFQDNDFPSLSGGRVVRIATHPDYQGMGYGSRAMSLLQKYYQGE FPSMAETPETKTITTVEKEAVGLLEEVIVPRKNLPPLLSKLSERPAEKLDYIGVSFGLTA DLLKFWRKAGYLPVYLRQTANDLTGEHSCIMLKTVQESDTEGANQETWLTAFWTDFRKRF MSLCAYQFRAFKPSLALSVLFCKAYNKMKPQPMTATELELLFSKYDIQRLELYSRNMADY 
bioRxiv preprint doi: https://doi.org/10.1101/2021.11.30.470322; this version posted November 30, 2021. The copyright holder for this preprint (which was not certified by peer review) is the author/funder. All rights reserved. No reuse allowed without permission.

HLITDLLPSVTRLVFLKKINFQLSAVQFSILLGLGLQHKTVDVLEKETEVPVSQLLALFN RTVRKVVTELNSVLEREVEKDMMEEKVVNMEPTRQTMAQELDEAAAVIEAKQKKELAALK GLDLSRYAIRGQESEWDDALKSGKKKNLISIKIANQEKRAAEKSKSTEKRKGDRESLGGG DDDQEMSKKKKKKKKFTPGKGSKN

$>$ A mellifera

MVRKKIDNRIRILIENGVITGYRTMFI I IGEKARDQVVLLHHMLSKSI IKARPSVLWCYK KELGFSSHRKKKMKSLQKKVKSGKLDVNEDDPFELFVVSTNIRYCYYHETHRILGNTYGM CVLQDFEALTPNLLARTIETVEGGGLIIFLLQSVNSLKQLYTMNMDVHQRFRTEAHQNII GRFNERFLLSLASCKRCLVVDDQLNVLPLSSHNLKIEPIQKLSSSETLSELDTLKESLKD TQPISSLVNCCKTLDQAKVLLKFIECISEKTLRSTVSLTAARGRGKSAALGLAVAAAITF GYSNIYISSPSPENLNTLFEFIFKGFDALGYQEHLDYGLVQSTNPEFNKAIIRVNIFRDH RQTVQYIHPTDAHKLNQAELLVIDEAAAIPLPYVKAMLGPYLIFLASTINGYEGTGRSLS LKLLQQLRNQTISSNSHEKQKNEKI I IGRQLHELTLEESIRYKPGDSIEQWLCDLLCLNV IINTPILSGCPSPDICELYYINRDTLFSYHKASELFLQRLVALYVASHYKNSPNDLQMMS DAPAHHLFCLLGPIDSNKKILPEILVVIQICLEGEINKNTINDGLVRGRRAAGDLIPWTI AQQYQDQDFPALAGARIVRIATHPEYQSMGYGSRALELLKKYYEMKIVNINETSLQTCMT EISKVKNEEVNLLEEKIEPRVSLPPLLLKLSERRPENLDYIGVSFGVTEPLLKFYKRANF IPVYLRQTTNDITGENSCIMLYKINSDQDITWLQAYWNDFRKRFISLLSFSFNTYSPSLA LSILFNKSITSEATTLTKNILDIYFTSYDLKRLSMYSNNMADYHLIMDLLPSLARLYFLN MMDDIHLSAVQSAILLGLGLQHKTVDTLAKELDLPTTQLLGLFNRIIRKFIQYLNKITEN FIETTMMKIEGNNETIQLNPINGKSLYDELENAAKELKAKQKAELEKLKKENLEQYAIKG TEADWNVALSEKKSKNLISIKSGEKRELEHNILEENKKQFKKKKKHFSKI

$>$ A_gambiae MVKKKIDNRIRVMIENGVKLGHRTMFVIVGNKARDQVPILYDILTKASVKARPTVLWCYK NKDEAISNHGKKRAKKIQSGKIDINESDLFDAFRVATTIHGRYYKDTHTILGKTYGVCVL QDFEALTPNLMARTVETVEGGGLIILLLKTISSLKQLYTMSMDVHKRYRTEAHQNVTCRF NERLILSLADCSRCLLVNDDLTVLPLSSRTADVKPIDVASIENVQSEQLAELKESLADAP PAGPLVNLCRTYDQAKAVAQFIDALAEKQLKPPTSLTAGRGRGKSAAMGLAIAGSIAFGY VNVYVTSPSPENLITLFEFILKGFDVLEYQEHTDYTIIRSTNPNFNKAIIRINITRNNRQ TIQYISPTDAHLLNAADLLIIDEAAAIPLPMVRAMLGPYLVFMASTINGYEGTGRSLSLK LLSQLQKETNAPLPIKLEESIRYSPGDPVESWLTSLLCLDATIVQNMNSGCPPPDECQLY YVDRDALFSYHKAAETFLQRIVSICVSSHYKNSPNDLQMMSDAPAHHLFCLLGPIVKKNQ LPEILVVIQVCLEGEISSTTLANSLVRGMKASGDLIPWNITEQFGDHEFPKLSGARIVRI ATHPNYQRMGYGKRALNQLKHYYEGRFPIESDGLGDEQGGDDNGIETIDDDEVDLLKEVI TPRKKIPTLLKALTERKPEMLDYLGTSYGLTGELLRFWKSQKFVPVYLSQKENELTGEHS CIMLCPINSTVERVETNEWLNDYFLDFRRRVLKLLGKSFNKFTTSLALSLLENKAVKMEG KALTQTTIDEVFLPHDVQRLEMYIHNQVEYKLIMDLTSDLASLYFQGKMSGAHIETLHKA ILLGCGLQHKTIDKLMEELNMPSNQVLAKFYDCVKKLTNYILRTMESTIESGMAKPSELN MGENLVPLKQSLDDEFAEDVKTLEKQQKKELAKLKKLNLDQYAIKGTDEEWSKVLSSNKS TIVSIKSGEKRLNENADDSMSAETGDVSAFQKKKAKFGKKQKSRNNKG

$>$ D_melanogaster

MVKKKIDNRIRVMIENGVKLGHRTMFIVIGDKARDQVPILYDILTKSTVKARPTVLWCYK NKDEAISNHGKKRAKKIAVGKVDVNEADLFDSFRVATTIHGRYYSETHAVLGRTYGVCVL QDFEALTPNLLARTVETVEGGGLIILLLKTLQSLKQLYTMSMDVHKRFRTEAHQTVTCRF NERLILSLADCKRCLVVNDDLTVLPLSSKTINVEPVNPAGAGRSPNEASLKELKESLLTV QPAGALVNLCKTYDQANAVAQFIEALVDKQLKPPMSLTAARGRGKSAALGLSIAAAVAFG YVNIYVTSPHPENLITLFEFVLKGFDALEYQEHADYTIIRSTNADYKKAIIRINITRSSR QTIQYIAPSDTHLLNAADLLLIDEAAAIPLPLVKKMIGPYLVFMASTINGYEGTGRSLSL KLISQLQKDNNARPPLKLEESIRYQENDDIEKWLINLLCLDASTVPSISSGCPTPDACEL YYVDRDALFSYHKAAEAFLHRLVSIYVSSHYKNTPNDLQMMSDAPAHHLFCLLGPVQRMD ALPEILVVIQVALEGQISAQSISDSLGRGKKAAGDLIPWNVAEQYGDRDFPKLCGVRIVR VATHPNYQRMGYGKRAIQLLKDYYARKHTNLEDGPVASKDAGKGIEEVEEEELSLLKEQI RPRSRIPTLLQRLHERVPEHVDYIGTSYGLTTELLKFWKNAGFVPVYLSQKSNELTAEHS CIMLHTPNATPWLGLYYQDFRRRVLKLMGKTFREFETKLCLALLKNKSVDTEGSALKVLD KPMLDVYFLPHDLQRLESYARQQSEFRLIIDLLTDIAQLYFQGRIDGLQLDLVQQGILLA LGVQGKTVDALGLELNMPGNQLLAKFFDAMKRCNQCFRSVLEEHIEGGMLREADLSKGEE LQPLTLSLDKELDQTAQKLSKQQRKELKRLKAEQLDEFQIKGTEEDWSKALETNGTGGGS GLLSVKSGVKRLDGPIETREDGDLAAPLSKKKKKNNPKQRRSQGKSLI

$>B$ mori

MVKKKIDNRIRVMIENGVKLGHRTMFLLIGDKSRDQVPILYDMLVKSTVKARPTVLWCYK NKDEAISNHGRKRAKKIAAGKLEVSEESLFDAFRVATTIHGRYYSESHAMLGQTYGVCVL 
bioRxiv preprint doi: https://doi.org/10.1101/2021.11.30.470322; this version posted November 30, 2021. The copyright holder for this preprint (which was not certified by peer review) is the author/funder. All rights reserved. No reuse allowed without permission.

QDFEALTPNLMARTIETVEGGGLIIFLLKTMDSLRQLHSITMDVHSRFKTEAYDTVVNRF NERFLLSLADNPRCLVLDDSLTVLPISSKTAQIDPVDATPERVNPKLTELISSLSDSPPA GPLVALTRTYDQATALIALINTLADKQSRPPHCLTAARGRGKSACLGLAVAAAVALGYVN IYVTSPHPENLITLFEFLLRGLDACLYQEHIDYNIVRSTNPDFKKAIVRVNIARNSRQTV QYITPDDHSLLSAADLVLVDEAAAIPLVHVAAAAQKAPLALLSSTVSGYEGTGRALSLKL FAQLQTQHNAPPPIKLDEPIRYRTGDPIESWLNSLLCLEAPPPSMGVGAPPPAACELYRV NRDALFCYHKAAEAFLHRLVSIYVASHYKNSPNDLQLLADAPAHCLFVLLAPPPRAAALP ELLCVLQLCLEGNISDKSVRDSLGRGRKAAGDLIPWNICEQFGDKDFPKLSGARIVRIAT HPAYQRMGYGKRALQQLAAYYSGDIPCLDEDASDAETDRQTDAQSTLQTETIAPRSKPPT LLKRLTEVRPENLDYLGTSFGLTEDLLRFWKSQKYVPVYLSQKANELTGEHSCIMLRALR DAAWLGAYSADFTRRAARLLPRTLRRLPAALALSTLVTDSVKTNKPVLTKDLIEQYLTGH DLSRLEAYSKQQADYRLITDLLQPLAELVFHTKSTIKLDAVQKVIFIAMGFQMKDVDDIA SELGLPGSQILAKFYEASKKINAALNAVLEDSAAKEIGIEDKAASGAAQPVQQSLQDELS KAAKDLERKQRKELSKLMGEDLSQYRIKGSDLDWGQALASTKQKTILSVKSGEKRLGEDM KEIDDLLDLQTNKKKKKKKSFKN

$>$ I_scapularis

MTRKKIDNRLRVLIENGVHTGHRTMFVVVGEKGRDQVVILHHMLSKTTVKARPSVLWCYK KELGFSTHRKKRMRQLQKKIKSGKLDVNEEDPFELFVAATNIRYCYYHETHKILGQTYGM LVLQDFEAMTANVLARTMETVEGGGLVVLLLQSISSLRQLYTLAMDVHARYRTEAHQEVV GRFNERFILSLSSCEACLVVDDRLNILPVSTSAAGIAPVPRAPQDPEASELASLRASLED SQPLGALVAVCSTCDQARALLKCVDAVAEKTFRTTVAVTAARGRGKSAALGLAVAAAVAF GYSNVFVTAPSPENLKTLFEFVLKGFDALKYEEHQDYEVVQSSQPELNKAVVRVTVFHEE HRQVVQWVHPGDSHLLGQAELLVVDEAAAIPLPLVQKLLGPYLVFLSSTVNGYEGTGRSL SLKLIQQLRQQSAAGDAGKSSSGGRVLHEVSLSESIRYRPGDPVEAWLNRLLCLDANVPP ASGCPPPGDCQLFYVNRDTLFSYHRASESFLQQVVSLCVASHYKNSPNDLQMLSDAPAHH LFVLLPPVQPDARALPQVLCVLQVCYEGGISRASVSDNFARGRRAHGDLIPWTVSQQFQD EEFAGLAGARIVRIATHPEYQRMGYGLRALELLTEYHLGHTPSLEEAEAATPGAAGADET GAEEAARERLEPRKRLPPLLLRLSEKRPERLDYLGVSYGLTADLLRFWKKAGYVPVYLRQ TPSELTGEHSTIMLRCLQEGAQWLPQLWADFRRRFLSLLGYQFRVLKPATALSVLRNPLE DSVHPRVPSLAEVEREFTAYDLRRLELYARNLVDHHLVTDLLPALARMHFLKALGDVHLS AVQSAILLGLGLQHRTVDELSRELDLPTSQLLGLFNRTVRKCATFLGALLEKSLDAALPR PSPALLPLQPVVQSLGDELSGAAREIEARQKKELKKLQQMDLSQYSIKGSDSEWKDALQQ PGKQLVSIKSINKRPQETDTPEKPQKKKKKQK

$>$ C_elegans

MRTKLDGRIRTQIENGVASGHRSMFAVVGDKARDQVPILYHILSKSTVSARPNVLWCYKK ELSFSTHRAKKAKKMKKATTTISGSLPDADPFDVFISSTQIRYCYYNETEKILGNTFGVL VLQDFEAMTPNLLARTIETIEGGGMVILLMQSVRSLRQLYTISMDVHNRYRTEAHNEITA RFNERFILSLASCSSVLVLDDQLRVLPISSHIENVEAIPASQKKIQSESDAELASLKEAM KETKPIGPLLSRARTACQAKALLRFLDVITEKQSNVTCSLTAGRGRGKSAAVGLSLAGAI AFGYTNIFVTSPSPENLKTLFEFVVKGFDALDYQEHTDYELIQSANPEFKNCLVRINVFR EHKQTIQYISPTDVQKLGQCELIVIDEAAAIPLPLVKELISGPYISFLSSTINGYEGTGR SLSLKLLQQLRQQSAGGEAKEGKSASNKGKTLHEMHMEESIRYKPGDKIEKWLNRLLCLD ATNCQLKLECGTPPPAACELYIVNRDTLFSFHDASEAFLQQVMAIFVSAHYKNSPNDLQM LSDAPAHNLFVLMAPIDKSRKTIPEVLAVVQVCLEGRLDSDNIQNGLESGKRAAGDLLPW TVSQQFMDKQFGTLCGGRIVRVAVHPDYQSMGYGGRAVQLIEQYYLGLATSLDEEEKAPA PPSKTVIKQVKDGHTVELLEERIEPRADLPPLLQRLDERKPERLDYLGVSFGLTVPLLKF WKRNEFVPVYIRQNSNDITGEHTCIILKGLEHGGSDSDEEPSATWLPVYWREFRRRIVNL LSFDFSSFPAQMALSLLQLKNKHVEKQMKRLVIERSELAIHLSNTDLRRMSQYGRNMVDS HIITDILPIVAKLNFEQRLPQELKLAVTQSAILLARGLQHKHFEDISKELDLPMNQIFAL LTKAIRRIGDWFDEVCETAVRENLDKEAEASAANKPTSSLPKAVPLANLEDELESAAKEI RARHDRDRKALLAELGNELQKYEIIQDEKELAEAYESVNMKYANKLVSVKSKRTAIQAAI PDAKDPANKNAKKKKRFSSGGRR

$>$ S_carpocapsae

MVRTKLDNRIRTLIENGVATGHRSMFAIVGDKGRDQIVILHHILSKAVVAARPSILWCYK KDLGFSSHRKKRMKEVQKKLALGKINVREDDPFELFVSSTEIRYCYYAESHKILGNTYGM LVLQDFEAITPNLLARAIETVEGGGIIVFLLRSVNSLRQLYTMTMDVHSRYRTEAHSEIV ARFNERFILSLASCKNCAVIDDQLNVLPISSHIKKIEAIPSSEKSKLTKEEEELKELKMA MADSKPIGQLLSKCRTNCQGKVLLRLLDVITEKSLKATCSITAARGRGKSAALGLAIAGA IGFGYTNVFVTSPSPENLKTLFEFIVKGFDAMDYEEHSDYELIQSTNPEFNKALVRINIT RDHRQTIQYFHPSDAGMLGQAELVVIDEAAAIPLPLVKELISGPYLVFLASTINGYEGTG RSLSLKLLQQLREQSSGAAKKTEKGGKLATATARMLHELSLEESIRYKPGDQIELWLNRI 
bioRxiv preprint doi: https://doi.org/10.1101/2021.11.30.470322; this version posted November 30, 2021. The copyright holder for this preprint (which was not certified by peer review) is the author/funder. All rights reserved. No reuse allowed without permission.

LCLDATNIHRLISGTPPPKDCELYYVNRDTLFSFHKGSEQFLRNMMAINVSAHYKNTPND

LQMLSDAPAHHLFVLMGPVRDEQTHLPEILAVVQVCIEGSLTKNSVTSSLERGRRAAGDL LPWTISQQFLDNDFPTLSGARIVRIAVHPDYQSMGYGSRALELLMQYYSGEVSSLSEKSL KSSASSKIRSVEDDKIGLLEEQIAPRPREDLPPLLHRLTERPAERLDYVGVSFGLTVNLL KFWKKASFVPVYLRQTTNDLTGEYTCIMLRSLAPQDDESSRDSWLASYWKEFRTRLISLL GFEFSKFSPHLAISLLQLKNPFVEKQMERKPYSRKELELFLSNTDLKRIAQYARNMIDHH LVTDLLPTVSKLYFNEKISDEVKINIVQSAILLGLGLQHKTVDRMVCELEMPANQILALF SKTMRRLSDHFDSMCMNALESQIGKKEQVVEGTPKGNVEMEAMPISLEEDLRDAEEDIKR RQKRDRAKLESELGKSFKEYAIKGTDEQWANALSTVKLDSAKKSAI ITVGSQMEKNSSAI EDLTKEAPRSAKKNLKRKRKSGGKL

$>$ M_galloprovincialis

MVRKKIDNRLRILIENGVAEKHRSMFVVVGDHGRDQVVILHHMLSKATVRARPSVLWCYK KELGFSSHRKKRMKQLQKKIKSGKLDVKEDDPFELFVASTNIRYCYYAETHKILGQTFGM CILQDFEALTPNLLARTIETVEGGGVVVILLKTMASLKQLYTMSMDVHARYRTESHQDVV GRFNERFLLSLTSCKNCMVIDDQFNMLPVSSHILNIQPVPAKSMEDALSPQEQELRDLKD SLQDTQPVGSIVNCCKTLDQAKAVLKFVEAISEKTLRSTAVMTAARGRGKSAALGLAIAT AVSFGYSNIFVTAPSPENLKTVFEFVFKGFDTLEYQEHLDYEIIQSTNPDFNKAIVRANI FREHRQTIQYIHPSDSHKLGQAELVCIDEAAAIPLPLVKNLLGPYLVFMSSTINGYEGTG RSLSLKLVQQLRQQSATFGGSSEVQKAISMATKSDSVSSATGRALHEIVLNDSIRYANGD PVEKWLNDLLCLDATNVSRITSGCPVPESCDLYYVNRDTLFSYHKASEGFLHRIMALYVS SHYKNTPNDLQLLSDAPAHHIFALLGPVDPNQSSLPEVLCILQICLEGDISSSTIMNSLS RGKRASGDLIPWTISQQFQDQDFAGLSGARVVRIATHPDYQSMGYGTRAMNLLQQYYKGE MISLSETKDIEDNVTPVDEQDVSLVDERLTPRKNLPPLLMKLTERKPEKLDYLGVSFGLT AGLLKFWKKSKFTPVYLRQTPNDLTGEHSCIMLKMLHTEDNPEDRSEPWLAAFWKDFRRR FVSLLSYQFRTFKPSMALNILQEKTFKSTGKKKMSLAELEAHFTKYDVKRLELYAQNMVD YHLVIDLLPAISRLFFLGLIDTHLSVVQSGLLLGLGLQHRTVDELEKELDLPSSQLLGLF NRI IRKIVQNLNEILEADVEKDMVARKEITLEPVKQTMDEELV

$>$ A_californica

MVRKKIDNRIRVMIENGVAQKHRSMVVLVGDRGKDQVVILHHMLTKATLKARPSVLWCYK KELGFSSHRKKRMKELQKKINSGKLSAKEDDPFELFISSTSIRYCYYAETHKILGNTYGM CVLQDFEALTPNLLARTVETVEGGGVIVLLLRTMSSLKQLYTMTMDVHLRFRTEAHQDVV GRFNERFLLSLASCETCVVLDDKLNLIPVSTHALALEPLPAKSLEENLTAQEIELEELKT SIQDMDVTTGKIIKLCRTLDQGQGFLKFVDAIVEKTLRSTVVMTAARGRGKSAALGLAMS ASVGFGYSNIFVTAPSPENLKTLFDFIFKGFDALDYQEHIDYEIIQSTNPDFNKAVVRVN IFRDHRQTIQYIHPSDAHKLGQAELVCIDEAAAIPLPLVKNLLGPYLVFMSSTVNGYEGT GRSLSLKLVQQLRQQVSTYGGSVKEARLAANMAKQGVDSSASGRVLHEVELKESIRYASG DPVEAWLNRLLCLDASSVPRSITGCPLPASCNLYYVNRDTLFSYHPASEKFLHRIMALYV SSHYKNSPNDLQLLSDAPAHHIFVLLGPVQSANTELPEVLCVLQVSLEGEISKSTIMNSL QRGQRGSGDLIPWTLSQQFQDQDFPGLSGARVVRIATHPDYQGMGYGSRALTLMQEYYEG KFPNLKESNPDDAGQESSNDGGDRGDEELKKVDDEEVTLLNEHLAPRKNLPPLLVKLTDR RPEPLQYLGVSYGLTSDLFRFWKRSGYVPVYLRQTANELTGEHSCIMVKTISVDEKEDKQ SWLPSFWKDFRKRLVALLAYQFRSFKPALALNILQNKNFKTEEKAVSLRRSELLQFVTTY DIQRLELYSSNMVDYHLIVDLLSDLARLFFTNHVPIHLSVVQSSILLALGLQHKTVEDVE KDIELPVSQILALLNRTIRKFVQHFNEILEKEVEATMAEKKEIVMEPTLTSVDQELKEAG QQFKQEHKQNVATLVGADLSHFAIKGSDKDWQEAIKGNSKSVISVKSHLEKKRKSPAEAD TDQMSNKKQKKRKKQREKS

$>$ A_californica

MVRKKIDNRIRVMIENGVAQKHRSMVVLVGDRGKDQVVILHHMLTKATLKARPSVLWCYK KELGFSSHRKKRMKELQKKINSGKLSAKEDDPFELFISSTSIRYCYYAETHKILGNTYGM CVLQDFEALTPNLLARTVETVEGGGVIVLLLRTMSSLKQLYTMTMDVHLRFRTEAHQDVV GRFNERFLLSLASCETCVVLDDKLNLIPVSTHALALEPLPAKSLEENLTAQEIELEELKT SIQDMDVTTGKI IKLCRTLDQGQGFLKFVDAIVEKTLRSTVVMTAARGRGKSAALGLAMS ASVGFGYSNIFVTAPSPENLKTLFDFIFKGFDALDYQEHIDYEIIQSTNPDFNKAVVRVN IFRDHRQTIQYIHPSDAHKLGQAELVCIDEAAAIPLPLVKNLLGPYLVFMSSTVNGYEGT GRSLSLKLVQQLRQQVSTYGGSVKEARLAANMAKQGVDSSASGRVLHEVELKESIRYASG DPVEAWLNRLLCLDASSVPRSITGCPLPASCNLYYVNRDTLFSYHPASEKFLHRIMALYV SSHYKNSPNDLQLLSDAPAHHIFVLLGPVQSANTELPEVLCVLQVSLEGEISKSTIMNSL QRGQRGSGDLIPWTLSQQFQDQDFPGLSGARVVRIATHPDYQGMGYGSRALTLMQEYYEG KFPNLKESNPDDAGQESSNDGGDRGDEELKKVDDEEVTLLNEHLAPRKNLPPLLVKLTDR RPEPLQYLGVSYGLTSDLFRFWKRSGYVPVYLRQTANELTGEHSCIMVKTISVDEKEDKQ SWLPSFWKDFRKRLVALLAYQFRSFKPALALNILQNKNFKTEEKAVSLRRSELLQFVTTY 
bioRxiv preprint doi: https://doi.org/10.1101/2021.11.30.470322; this version posted November 30, 2021. The copyright holder for this preprint (which was not certified by peer review) is the author/funder. All rights reserved. No reuse allowed without permission.

DIQRLELYSSNMVDYHLIVDLLSDLARLFFTNHVPIHLSVVQSSILLALGLQHKTVEDVE

KDIELPVSQILALLNRTIRKFVQHFNEILEKEVEATMAEKKEIVMEPTLTSVDQELKEAG QQFKQEHKQNVATLVGADLSHFAIKGSDKDWQEAIKGNSKSVISVKSHLEKKRKSPAEAD TDQMSNKKQKKRKKQREKS

$>0$ bimaculoides

MTRKKIDNRIKTLIENGVAKKHRTMFVVVADQGHDQIVVLHHMLTKAEVKARPSVLWCYK KELGFSSHGHKKRMRKMQKKIKAGRIATMSEKDADAFELFLASTNIRYCYYKETHKILGN TYGMCVLQDFEALTPNLLARTVETVEGGGLIVILLRTMNSLKQLYTMTMDVHSRFRTESH QDVTGRFNERFLLSLTTCSQCLVIDNQYNILPLSSLTSTVTPLPKLSKEESLTQSERELV ALKESLQDTQPVGAI INCARTLDQAKGLLKFIDSVSEKTLRNTVVMTAARGRGKSAALGL AMATAVGFGYSNIFVTSPSPENLKTLFEFIFKGFDVLEYQEHIDYEIIQSSNPDFNKAIV RVNIFRDHRQTIQYIHPTDAHKLGQAELVCIDEAAAIPLPLVKELIGPYLVFMSSTVNGY EGTGRSLSLKLIQQLRQQAATFSGTEVKKVTTVESTTNSLSGRALYEVQLNESIRYADGD PVEKWLNDLLCLDVTNVRRITSGCPLPQDCDLYYVSRDTLFSFHKGSEAFLHRIMSLYVS SHYKNTPNDLQMLSDAPAHHIFVLLGPMESTKQALPEVLCVVQMCLEGDISKAAVMSGLR QGKRASGDLIPWTISQQFQDNDFASLSGVRVVRIATHPDYQKMGYGSRALQLLSQYYKGE IVGLNEDVEEVPKDIEPVDEEELDLIVEKPVPRKNLPPLLLKLTERPPEQLDYMGVSFGL TADLLKFWKRNGFVPVYLRQTANDLTGEHSCIMLKTLHDKEDESNTLYDKKWLLSFWKDF RRRFISLCSYQFRFFSCSTALSILQQKHCKEEAVALAKQELDFKLTVFDLKRLELYSQNM IDYHLIMDLMPTIAELYFLQRLPELHLSAVQQALLLGIGLQHKTVDDLEKNLGLAASQLL GLYNRI IRKVVQHLNSIVEKSVEQTLVKEHKITMEPVPQSLDDELDAAAVEIKEEMRKKI APILEESELSQFAVKGDEDQWNLALSTGKKNFKSISIKSIRQKRQLKEVEENTSQKKSKK FKKSKKHK

$>$ L_anatina

MVRKKIDNRIRIMIENGVAERHRSLFVIVGDHGRDQVVILHHMLSKAAIKARPSVLWCYK KELGFSSHRKKRMRQLQKKIKSGKLDVKEDDPFELFVAATNIRYTYYSETHKILGNTFGM CVLQDFEALTPNLLARTVETVEGGGLVVLLLRTMTSLRQLYTMTMDVHARFRTESHQDVV GRFNERFLLSLASCKRCMVIDDQLNVLPVSSHTLQIQPVPPKTSDDPLPPSEQELKELKE SLQDTQPVGVLVDSCKTLDQAKAVLKFVEAISEKTLRSTVSLTAARGRGKSAALGLAIAA AVGFGYSNIFVTSPSPENLKTVFEFVFKGFDSLEYQEHLDYELVQSTNPEFNNAIVRVNI FREHRQTIQYIHPGDAHKLGQAELVCIDEAAAIPLPLVKNLLGPYLVFMASTVNGYEGTG RSLSLKLLQQLRQQNVTFGKQATDQQKGAPGGLASATGRILHEISLTESIRYGDGDAVEQ WLNEVLCLDASNVQRISSGCPVPENCDLYYVNRDTLFSYHKASEAFLQRIMGLYVASHYK NTPNDLQMLSDAPAHHIFVLLGPVDPTQNTLPEVLCVLQVCLEGEISKSNIMNSLSRGKR ASGDLIPWTISQQFQDHDFPQLSGGRVVRIATHPDYQGMGYGTRALEILQHYYEGKIPSL VEDAVVEEAVPTVSKDAVGLLEERIAPRKNLPPLLLKLSERPAEKLDYLGVSYGLTAGLL KFWKRSGFIPVYLRQTPNDLTGEHSCIMLKVLNEGEKTEQEEESWLMAFWKDFRRRFLSL LSYQFRTFHPSLSLSILQQKQCKEKESVISAEELDNYFTKYDLKRLELYSQNMVDYHLIM DLLPSIARLHFLNKTGVHISAVQAAILVSMGLQHKSVEDVEKDMEVPSSQLLGLFNRVIR KMVQQFNQIAEEAVEKDMVARKEVIMEPVKNSLDQELGEAAAEVAKKQKKDLELLKDADL SQYTIKGSEGDWENALTKGTKGLLSVKSGVQEKRKSVDSTSFEQQTPGKHKKHKKQKHHK $\mathrm{H}$

$>$ N_vectensis

MVLKKVDNRLRILIENGVQTRHRSLFVVVGDKGRDQVVILHHMLSKAAVKARPSVLWCYK KELGFSSHRKKRMKQLQKKIKSGTLDMNKDDPFELFVSSTNIRYCYYKETHKILGNTYGM CVLQDFEALTPNLLARTVETVEGGGIVVILLRTMSSLKQLYTMSMDVHARYRTESHQDVV GRFNERFILSLSSCKSCMVIDDQLNILPLTSHSLNIKAVPPKPKEESLTPSEVELKELKD SLQDTQPVGSLINCCRTLDQAKALLKFIEAISEKTLRTTVSLTAARGRGKSAAIGLALAS AVAFGYSNMFVTSPSPENLHTLFEFVFKGFDALDYQEHIDYELVQSTNPEFNKAVVRINI FRDHRQTIQYIHPSDAHKLGQAELLCIDEAAAIPLPLVKNLLGPYLVFMSSTINGYEGTG RSLSLKLLQQLRQQSVPQGVTSTKTAEAGGATTAVMGRMLSEVTLEESIRYAPGDAVEKW LNKLLCLDATVAQNITGGCPLPESCELYYVNRDTLFSYHKASEVFLQRLMALYVASHYKN TPDDLQLLSDAPAHHIFCLLGPVDPSSPALPEVYCVLQVCLEGDISKSTIMSSLSRGKRA SGDLIPWTVSQQFNDHEFASLSGGRVVRIATHPDVQGMGYGRKALSSLVQYYEGKIPNLS ENGQTETSHLGIPTESEADNLLAESITPRANLPPLLLKLGERPAERLDYLGVSYGLTAQL LRFWKRSCFVPVYLRQTANDLTGEHSCIMLRILGEIDAEQSDDWLKAYTVDFRRRFLSLL SFQFRSFPASLGLSILQDKSLKDHNQVLSRAELDFTLTNYDMKRLDMYSRNMVDHHLIMD LVPAVARFYFLRKLDISLSAIQSAILLGVGLQHKTFDQLEKELELPVGQLLGLFNRITRK VVQHLNSVVEAAIEGEIVKQKDVHMEPLAKSLNQDLEEAAKEMEVKQKKELEKLKLVDFS EYAISGKEDDWQEALSGKGTHNLVSIPSSKRKSLPTSDSGQQKKHKKSKKHRHSTK $>$ D_gigantea 
bioRxiv preprint doi: https://doi.org/10.1101/2021.11.30.470322; this version posted November 30, 2021. The copyright holder for this preprint (which was not certified by peer review) is the author/funder. All rights reserved. No reuse allowed without permission.

MVLKKIDNRIRVLIENGVQLRHRSLVFIVGDKGRDQVVILHHMLSKATIKARPTVLWCYK KELGFSSHRKKRMKQLQKKIKSGTLDLNKDDPFELFISSTNIRYCYYNETHKILGNTYGM CILQDFEALTPNLLARTVETVEGGGIIVVLLRTMTSLKQLYTMTMDVHARYRTESHQDVV GRFNERFILSLSSCKQCMVIDDRLNVLPISSQTLKITPLTPVTKEESVSPNDVELRELKS SLEDTPPVGVIVNCCKTLDQAKAVLKFIEAISEKTLRTTVALTAARGRGKSAALGLAMAA AIAFGYSNIFVTSPSPENLHTLFEFVFKGFDALDYQEHLDYELVQSTNPEFNKAVVRVNV FREHRQTIQYIHPGDSQKLGQAELVVVDEAAAIPLPLVKSLLGPYLVFLSSTINGYEGTG RSLSLKLISQLRVQSSGLGTRTTSESKNSNDTNTGSSASRILSEVSLNESIRYSPGDAVE DWLNHLLCLDATIVPKFSSGCPLPDACDLYYVNRDTLFSYHKASEVFLQRIMSLYVSSHY KNTPNDLQLLSDAPAHHIFCLLGPVDPAKKTLPEVFCVIQVCLEGEISKSSIMNSLARGK RASGDLIPWTISQQFNDYEFPSLSGGRIVRIATHPDYQGMGYGKRALQLLIQYYEGKIAD LSESTVQPTDQEIQPTDLNESGDLLKETIGPRNNLPPLLLKLNERCAERLDYLGVSYGLT NDLLRFWKKSSFVPVYIRQTANELTGEHSCIMLRDLHHVDENGTSGWLLAFWTDFRRRFL SLLSFQFGLFSATLALSVLQNKVFVGSNQGLTQSELYSVLTKYDIKRLDMYSKNLVDYHL ITDLLPAVSRLFFLQKTNFHLSGVQSAILMGIGLQHKTMEDLEKELQLPVSQLLGLFSRV IRKVVKNFNEILESAVESEMTKEKEI IMEPLAKDLNEELEEIAEEIKEKQKEKTEMLKSM DLSQYAIAGKDNDWNEALKGKGPSQMISIASDKPEKRKSLALEESTSSKNKRKKQKKVRT G

$>$ E_muelleri

MSRKKVDNRIRVLIENSVALRHRSMFVVVGDKAKDQVSSQPRGSFVATANTIAFNSSSSH RKKRMRQLQKKLKSGTLDTKDDPFELFVAATTIRYCYYSETHKILGSTYGMCVLQDFEAL TPNLLARTIETVEGGGMVVFLLKSMASLKQLYTLTMDIHARYRTEAHNDVVGRFNERFLL SLGDCSHCMVIDDQLNLLPVSSHMLKLEPVRAKTKEESMTPEQLDLQALKHSLADTQPVG VLVECAKTLDQAKALLQFVEAISEKTLRTTVALTASRGRGKSAALGMAIAAAVAFGYSNI FVTSPSPENLKTVFEFVLKGFDALHYVEHEDYEVIQSTNPEFNEAVVRVNIFHEHRQTIQ YIHPADSHKLEQAELVCIDEAAAIPLPLVKNLLGPYLVFMASTINGYEGTGRSLSLKLID QLRQQSSVTGVSASSSGGASFAGRTLREVTLEEPIRYSAGDPVERWLHGLLCLDASSIRR VGVGCPPPQDCELYHVNRDTLFSYHKASEEFLHRLMALYVASHYKNTPNDLQLLSDAPRP PRLCPPWARGPFHVLPARDPVCAAAQFVYVDVSSCDLTRQFADNNFASLSGARVVRIATH PDFQSMGYGSRALSLLSDYYEGRFPSLSEEADRDQSGVVNVALDNDEEGEDGGLLMEGGG ATGQAAPAVDELDGAEAQRGWTKPGRGIWNDGKLFKVAPHGSCGFWKKAGYLPVYLRQTE NELTGEHSAIMLKALASSSSGSSDWLKAYTIDFRGRFASLLSYQFRQFKPVVALSVLHTA SCFDTRSACAYEELTRVLSQQDLKRLDLYSRNMADHHLITDLLPTLAKLYFWSKVPFNLS AVQASILLGMGLQHKTVEVLEKELGLPSSQIMGLFNRSVRKFSSHFTEVVEGVVGRGIPL PTDAGMAPLEHTVDQELSEEVKAFKKKQLDLKEQLETADLEQYSVSGKEVDWAGVTTDKP GIISMARTGHKRLPTKPSHKEKEGKAKRKKQK

$>$ U_maydis

MRKQLDPRIPTLIRNNVALNHRSFFVIVGDKGKDQIVNLHFLLSQSRVQSRPNVLWCYKK DLGFTTHRKKREQKIKNDIKRGIREKGQGDPFELFVSLTDIRYCYYKDTPKILGQTYGML ILQDFEAVTPNMLARTIETVEGGGVVILLLKTMSSLRQLYSLGMDVHRNYRSNASDDDPV ARFNERFLLSLGANPDTLLLDDELNVLPLSKAKDIQPLPETSTGAGTGVGSTVRKGKERV EADEELAELKDQVRETKVVGQVVRHAKTLDQAKAVLTILDILASSSLSTTVALTAARGRG KSAALGLCIAAAVAHGYSNIFVTSPSPENLKTLFEFVFKGLDALGYDEVADWDLQRGTGE WKDVVVRVNIFRGHRQTIQYIQPQDHQVLGQAELVVIDEAAAIPLPLVRNLMGPYLVFLS STINGYEGTGRSLSLKLIQQLRDSARGIADATDADDSTASSSKAVRKQAKGGLNTGRAAG AALAARSLKEVELKEPIRYSRGDKIESWLHQLLCLDASLTRLSSAALKAKGCPHPSSCDL YMVNRDALFSYHPASEVFLQRMMALYVASHYKNSPNDLQLMSDAPGHRLFVLLAPLKGNE GGLPEPLCVVQVALEGNISRGVVLNSLSRGTREAGDLIPWLVAQQFQDADFASLSGARVV RIAVHPDYARMGYGARALQALEAFYSGQLLDVDNVRDDLDDGETFAAVRDRKISKDANLL QGDEIRVRDAARMPALLQRLSERRPEQLDWLGVSYGLTPQLFKFWKKAGYTPLWVRQIAN DLTGEYTTVQLKSLDTSISTTGSAWLGSLAADFRKRFISLLSYRFREFSTITALTVLEAA TQGARLAADDDSLPASSTSLSLGAAELRTLLTPFDMKRLDSYSNNMVEMSVVLDLLPTLG ALYFNNRLRAVREDEATASAIDAQDDEEELRLSGLQSSLLLAIGLQRKTPDEISAELRLP LQQAMALFVKTVRLLVKSLRKVEKRDIVRSMPELSSGLEARAPLRKKANSARGTDQSDDW TALKGDLQSELRDAGREFLAGNKESEAYGKLDDDQEEDDHESKDGADQQEDKEEEGEEQD DVDEDDDDDDDDDELLTAKQKLIDSMDLSKYAIKDGADGANWSQAEAEVANMLRKNGGND LKGFNTTISVKGTKRAADDKVEASPKKAKASNKSGERKKQKRR $>$ S_pombe MPKKALDSRIPTLIKNGCQEKQRSFFVVVGDRARDQVVNLHWLLSQSKVAARPNVLWMYK KDLLGFTSHRKKRENKIKKEIKRGIRDPNSEDPFELFCSITNIRYCYYKESEKILGQTYG MLVLQDFEALTPNLLARTIETVEGGGIVVLLLHKLNSLKQLYTMSMDIHSRYRTEAHSDV 
bioRxiv preprint doi: https://doi.org/10.1101/2021.11.30.470322; this version posted November 30, 2021. The copyright holder for this preprint (which was not certified by peer review) is the author/funder. All rights reserved. No reuse allowed without permission.

TARFNERFILSLGNCENCLVIDDELNVLPISGGKNVKALPPTLEEDNSTQNSIKELQESL GEDHPAGALVGVTKTLDQARAVLTFVESIVEKSLKGTVSLTAGRGRGKSAALGLAIAAAI AHGYSNIFITSPSPENLKTLFEFIFKGFDALNYEEHVDYDIIQSTNPAYHNAIVRVNIFR DHRQTIQYISPEDSNVLGQAELVVIDEAAAIPLPLVRKLIGPYLVFMASTINGYEGTGRS LSLKLLQQLREQSRIYSGSGNNKSDSQSHISGRTLKEISLDEPIRYAMGDRIELWLNKLL CLDAASYVSRMATQGFPHPSECSLYRVSRDTLFSYHPISEAFLQRMMSLYVASHYKNSPN DLQLMSDAPAHQLFVLLPPVDLKNPKLPDPICVIQLALEGSISRESIMNSLSRGQRAGGD LIPWLISQQFQDENFAALGGARIVRIAVSPEHVKMGYGTRAMQLLHEYFEGKFISASEEF KAVKHSLKRIGDEEIENTALQTEKIHVRDAKTMPPLLLKLSELQPEPLHYVGVSYGLTPS LQKFWKREGYCPLYLRQTANDLTGEHTCVMLRVLEGRDSEWLGAFAQNFYRRFLSLLGYQ FREFAAITALSVLDACNNGTKYVVNSTSKLTNEEINNVFESYDLKRLESYSNNLLDYHVI VDLLPKLAHLYFSGKFPDSVKLSPVQQSVLLALGLQYKTIDTLEKEFNLPSNQLLAMLVK LSKKIMKCIDEIETKDIEEELGSNKKTESSNSKLPEFTPLQQSLEEELQEGADEAMLALR EKQRELINAIDLEKYAIRGNEEDWKAAENQIQKTNGKGARVVSIKGEKRKNNSLDASDKK TKEKPSSKKKFRK

$>$ A_bisporus

MRKQLDPRIPILINNGVKKNHRSFIVLVGDKGRDQIVNLHYLLSQARVSARPSVLWCYKK ELGFTSHRKKREAKIKRDVKRGIREPNEQNPFEIFVTVTDIRYTYYKESHKILGNTYGMC VLQDFEAITPNLLARTVETVEGGGLIVLLLKTMSSLKQLYTMTMDVHSRYRTSSHDSVIA RFNERFILSLGSCDDCLFLDDELNVLPISRGKDITPLSDTPGKEKEISSELKDLKDSLAD TKPAGDLVKLSKTIDQAKAILTFIDAIAEKTLSSTVTLTAGRGRGKSAALGLAMAAALAH GYSNIFVTSPSPENLKTLFEFVFKGLDALGYEEHLDYDIAQSTNPDFNKAIVRVNVFRGH RQTIQYIQPEDAHVLGQAELVVIDEAAAIPLPLVRNLIGPYLVFMASTINGYEGTGRSLS LKLIQQLRESTRPSLTKDLAAGTSKDDDSTAATTIASSSKKPLSKAPPKSRTLREIKLEI PIRYANGDKIEKWLNGLLCLDAAIVPKSIGQGCPHPSKCELYYVNRDTLFSYHPASELFL QRMMALYVASHYKNQPNDLQLMSDAPAHHLFVLLPPLKDDNDGGSGGVGNETYLPEPLVV IQVALEGSISKNVILEGLGRGLRAGGDMIPWLISQQFQETRFGMLSGARVVRIACHPDYA NMGYGSRALQTLNSFYSGELFNLDESHTVQSELPYPDALVNIDPSDSLLTEKPSIRAVNS MPPLLQRLTERKPENLDYLGVSYGLTSPLLRFWKRAGYVPLYLRQTQSDLTGEHTCVMVR GLNSSTEPELEWLSEFTKDFRKRFVSLLSYKFREFGSVTALSVLEAAHSGIRAKEGESKD EIQANELSLLLSPFDSKRLESYANNMLDYHVILDLMPIVAGLYFLKRLGEEVKLTAVQSA ILLAVGLQRKSVEDVETELQLPVSQALALFAKLIKKISKRLVDIQKAAISAELPLQSTGV VGISRADDGTKEIKEKKATEGWKSVGEVLEDELNEAGDEATKRLREKQREMIDALDLQKY TIDDVEMDWSSAEAQIRGGGTNKGYSTVVSIKSGAEGGKSSGQKRKAVEEAEDDSKSGKE AKKTRRGKNRSTSLRLGK

$>$ N_crassa

MSQKVIDSRIPALIRNGMQEKKRSFFVVVGDHSKEAIVHLYYILSNANMKHNKSVLWAYK SKLLGFTSHRKKRENKIKKEIKRGVREANSEDPFELFISLNDIRYVYYKETEKILGNTYG MCILQDFEAMTPNILARTIETVEGGGLVILLLKGMNSLKQLYTLSMDVHSRYRTEAHDDV VARFNERFILSLGSCESCLVVDDELNVLPISGGKAVKALPRPTDDEEVNPAKKELEKIKE SLEDTQPIGSLVQLARTTDQAKALLTFVDAIAEKTLRNTVTLTAARGRGKSAAMGVAIAA AVAYGYSNIFITSPSPENLKTLFEFVFKGFDALDYKDHADYSIIQSTNPEFNKAIVRVNI HRNHRQTIQYIRPQDAHVLGQAELLVIDEAAAIPLPLVKKLMGPYLVFMASTISGYEGTG RSLSLKLIKQLREQSRTGAGVKDAVEVDRTSGKAAKDTETVFTGGRSLKEITLSEPIRYA QGDPVEKWLNTLLCLDATLPRSKLNTQGCPDPSQCELLHVNRDTLFSFHPVSEKFLQQMV ALYVASHYKNSPNDLQLMSDAPAHELFVLTAPIVEGRLPEPLCVIQVSLEGKISKQSILN SLSRGSQPAGDLIPWLVSQQFQDDEFASLSGARVVRIATNPDYVSMGYGSRALQLLVDYY EGKFANLSEEAGTVVPKSAPRVTDAELANASLFDDIKVRDMNELPPLFAKLAERRPEPLE YIGVSYGLTQPLHKFWKRASFAPVYLRQTPNDLTGEHTCVMLRPLGDGTDPSWLGAFAND FHRRFLSLLSYKFREFPAILALTIEESASVGAKLDPSNEPTPLEKAELDKLLTPFDHKRL ESYANGLLDYHVVLDLIPSIAQLFFEGRIRSNLQLSGLQQAILLAIGLQRKEIDAIAGEL TLPSSQLLAIFIKIMRKISTHLASLVEGAIAKEMPQQIGVSAENASAAHDDEIIENNFVP LDQNLDDELAEGGDQAMKAIRAKQRELIDSLPLDQYEIDGGDAEWKEAEKRVAKATKEGK SNPLVSVKTKKRKAEPVYEEEKSEKRDRGDKKGKKVRHDKRK

$>P \_$anserina

MFSYLLLAYIAHLDQYQLTFLPHRDSRIPTLIRNGLQEKKRSFFVVVGDHAKDAIVHLYY IMSSMEVRHNKQVLWAYKNKLLGFTSHRKKRENKIKKEIKRGIREANSEDPFELFISLND IRYVYYKETEKILGNTYGMLILQDFEAMTPNILARTIETVEGGGLVVLLLKGMNSLKQLY TMSMDVHSRYRTEAHDDVVARFNERFILSLGSCDSCLVIDDELNVLPISGGKGVKALPPP EEDEPLSKTKLELEKIKDSLQDTQPIGSLVKLAKTTDQAKALLTFVDAIAEKTLRNTVTL TAARGRGKSAAMGVAIAAAVAYGYSNIFITSPSPENLKTLFEFVFKGFDALDYKDHADYS 
bioRxiv preprint doi: https://doi.org/10.1101/2021.11.30.470322; this version posted November 30, 2021. The copyright holder for this preprint (which was not certified by peer review) is the author/funder. All rights reserved. No reuse allowed without permission.

IIQSTNPDFNKAIVRVNIHRNHRQTIQYFRPQDAHVLGQAELVVIDEAAAIPLPLVKKLM GPYLVFMASTVSGYEGTGRSLSLKLIKQLREQSMTGGNPNGSGTAEVDRASGKTAKETAG IGGRSLKEITLSEPIRYAQGDSVEKWLNTLLCLDATLPRSKLSTQGCPDPSQCELLHVNR DTLFSFHPVSERFLQQMVALYVASHYKNSPNDLQLMSDAPAHELFVLTGPISEGRLPEPL CVIQVSLEGKISRQSILNSLARGSQPAGDLIPWLVSQQFQDDEFASLSGARVVRIATNPD YVSMGYGSKALELLVSYYEGKFANLSEDDDVTMAQAAPRVTRAELERGSLFDDIKVRDMS ELPPLLPNWQRG

$>$ A_niger

MPRKAIDSRIPALIRNGVQEKKRSFFVVVGDRAKDVIVHLHYIMSSVDVKQNKSVLWAYK KDLLGFTSHRKKREAKIKKEVKRGIREPNQEDPFELFITLNQIRYVYYKETEKILGNTYG MCVLQDFEAMTPNLLARTVETVEGGGIVLLLLKGMNSLKQLYTLSMDIHSRYRTEAHDDV TARFNERFILSLGSCDSCLVVDDELNVLPISGGKSVKPLPPPESIDDTNIGTKKELKEIK ESLAESQPIGSLVNLARTVDQAKALLTFADAIAEKTLRSTVALTAARGRGKSAALGVAIA AAVAHGYSNIFITSPSPENLKTLFEFVFKGFDALGYLDHVDYTILQSTNPDFNKAIVRVN IHRQHRQTIQYIQPQDAHVLGQAELLVIDEAAAIPLPLVRKLMGPYLVFMASTINGYEGT GRSLSLKLIQQLREQSRGGVKVSGEDDIDVADRATGKAAKNIDKSLAGRSLREITLSEPI RYAPGDSVEKWLNKVLCLDATLPKSRMNTQGTPHPSQCQLLQVNRDTLFSFHPVSEKFLQ QMMALYVASHYKNTPNDLQLMSDAPAHQLFVLVPPIDEEATKLPEPLCVIQVALEGRISR QSVLNSLSRGQRAGGDLIPWLVSQQYQDEDFAGLSGARIVRIATNPEYLSMGYGSRALEL LIDFYEGKFTSLSEDIPEPQEEMVRVTDEELTNSNLLDDNVKVRDIHTMPPLFGKLSERR PDALDYVGVSFGLTPSLHKFWKRSSFSPVYLRQTPNDLTGEHSCVMLRTLTSGSNDAAWL GAFSRDFHKRFSSLLSYQFREFPSVLSLSICESANSGAKLDPSISQSPLTKAELDAAFSP FDLKRLDSYANNLLDYHVILDMVPTIAEYYFSGRLSGKVSLSGVQQSILLAIGLQRKNLD TIEKELTLPSSQLLAMFLKIIRKVSTYFRNLIEGAVAETMPAEKAPSARVTTEAHDELPD ERFKPLETSLDDELREGGQEVNEELREKQRALIDALPLDKYEINHGSAAWEEAEKQIRSG GASTVSVKTSKQTKRKKGESAREIYDQEIDSKRQKI IKKGTEGKKKKH

$>$ S_cerevisiae

MAKKAIDSRIPSLIRNGVQTKQRSIFVIVGDRARNQLPNLHYLMMSADLKMNKFVLWAYK KKLLGFTSHRKKRENKIKKEIKRGTREVNEMDPFESFISNQNIRYVYYKESEKILGNTYG MCILQDFEALTPNLLARTIETVEGGGIVVILLKSMSSLKQLYTMTMDVHARYRTEAHGDV VARFNERFILSLGSNPNCLVVDDELNVLPLSGAKNVKPLPPKEDDELPPKQLELQELKES LEDVQPAGSLVSLSKTVNQAHAILSFIDAISEKTLNFTVALTAGRGRGKSAALGISIAAA VSHGYSNIFVTSPSPENLKTLFEFIFKGFDALGYQEHIDYDIIQSTNPDFNKAIVRVDIK RDHRQTIQYIVPQDHQVLGQAELVVIDEAAAIPLPIVKNLLGPYLVFMASTINGYEGTGR SLSLKLIQQLRNQNNTSGRESTQTAVVSRDNKEKDSHLHSQSRQLREISLDEPIRYAPGD PIEKWLNKLLCLDVTLIKNPRFATRGTPHPSQCNLFVVNRDTLFSYHPVSENFLEKMMAL YVSSHYKNSPNDLQLMSDAPAHKLFVLLPPIDPKDGGRIPDPLCVIQIALEGEISKESVR NSLSRGQRAGGDLIPWLISQQFQDEEFASLSGARIVRIATNPEYASMGYGSRAIELLRDY FEGKFTDMSEDVRPKDYSIKRVSDKELAKTNLLKDDVKLRDAKTLPPLLLKLSEQPPHYL HYLGVSYGLTQSLHKFWKNNSFVPVYLRQTANDLTGEHTCVMLNVLEGRESNWLVEFAKD FRKRFLSLLSYDFHKFTAVQALSVIESSKKAQDLSDDEKHDNKELTRTHLDDIFSPFDLK RLDSYSNNLLDYHVIVDMIPMLALLYFGDKMGDSVKLSSVQSAILLAIGLQRKNIDTIAK ELNLPSNQTIAMFAKIMRKMSQYFRQLLSQSIEETLPNIKDDAIAEMDGEEIKNYNAAEA LDQMEEDLEEAGSEAVQAMREKQKELINSLNLDKYAINDNSEEWAESQKSLEIAAKAKGV VSLKTGKKRTTEKAEDIYRQEMKAMKKPRKSKKAAN

$>D$ discoideum

MVRKKVDTRIRTLIENGVATNHRSFFVIVGDNGKDQVPNLHYILSKSIVKARPSVLWCYK EDLGFSKHKKKKMKLKEKSKSSGIDSVNQEDPFDVFISTTNIRYSYYSESHKILGNTFGM LVLQDFEAITPNLLARTIETVEGGGLIVLLLKTMDSLKQLYTMTMDVHTRFRSENSKGEV VCRFNERFLLSLGKSEQCLVMDDELNILPISSQSRSIEAKQQILETPEQVELREFKQQVK DTDIAGALIENTKTMDQATALLTFIDSISEKTLRSTVTLTAGRGRGKSAALGLAISAAVA FGYSNIFVSSPTPENLNTLFQFVFKGFDSMEYVEHVDYELVKSTNPEFHDAI IRVNIFRS HRQTIQYIQPQDYQKLGQAELVVIDEAAAIPLPLVKNLLGPYLVFMSSTINGYEGTGRSL SLKLIKQLREQSTVVSNSSNKALNSITGRMLREIELNEPIRYSARDPIERWLNELLCLDS TIAKSSPTGCPHPSACQLYYVNRDTLFSYHKASEAFLQKMVGLFVSSHYKNSPNDLLLMS DAPDHHLFVLLGPIDENNTTGLPEILCAVQVSLEGEIAKESILNSIKRGYQASGDLIPWT LTQQYQDEDFPRLSGVRIVRIATHPDYQKMGYGSKALELLTQYYQGEITNLDEVNQEEEE EDEEKVDQNKGSSKISTVKKDNASLLTEVIRPKSNIPPLLFKLSERKPEKLHYMGVSYGL TQQLYQFWSKSKYLPVYLRLTSNDITGEHTCIMLRELNNEQNNTICKDGWLQSFHQDFKK RFINLLGYEFRNFNSSIALNILYEKKVNNTVAVASSTTKNELTQNEMELLFSSYDLKRLE SYSNNIVDYHVVIDLLPSLSKLYFTNKLKIEDISLIQSAILLALGLQHKTVDNLIGELNL 
bioRxiv preprint doi: https://doi.org/10.1101/2021.11.30.470322; this version posted November 30, 2021. The copyright holder for this preprint (which was not certified by peer review) is the author/funder. All rights reserved. No reuse allowed without permission.

ASNQVLSLFNQTMRKINTELKQKQEKFIQDSMPKLSIVAPRTGQFKSFKGDIKETDMIPL

AEDMESELEKGAEEVVNKLKHQLENDSSLSKYLVKGNDEDWSKALKPGSIPNSITIKRKS DENEETDKKENNKKSKTKNNNNNNNNNKKVNNQKSKK

$>$ P_polycephalum

MVKKKVDSRLRTLIENGVKTGHRSMFVIVGDNARDQVVNLHYLVSKASVQARPSVLWCYK KDLGFSTNREKRMKQRKAMFRKGQADPDMNDPFDLFISSTSIRYTYYSESHKVLGNTYGM MVLQDFEAITPNLLARTIETVAGGGVIVVILKTMTSLKQLYTMTMDVHNRFRQDESEDVV GRFNERFLLSISVCPTCLVIDDELNILPVSSFART IKPIEGRMVGDETIAQRELRELKNS LKDVHPVSELVGVAKTVDQAKAIMTFVESITEKTLRSTVVLTAARGRGKSAALGVAMAAA IAFGYSNIFVTSPSPENLATLFEFVFKGFDALEYKEHIDYELLRSTNEQFNKAIVRVNIF KSHRQTIQYIQPQDYQKLGQAELVVIDEAAAIPLPFVKNLLGPYLVFLSSTINGYEGTGR SLSLKLIKQLREGSAAGNSGGRVLREMKLTEPIRYTPGDEVEKWLNGLLCLDATVAPPIA TGYPDPAVCQLYYVNRDTLFSFHKQAETFLQRMVALYVSSHYKNSPNDLLLMSDAPGHHL FVLLGPVDVTKNALPEIMCVIQVALEGRIVKDAVIAGLARGVRSAGDVIPWTISQQFQDY EFGRLSGARIVRIATHPDYHRMGYGTRALELLASYYQGDIISLNEEDEKEEEEEEEEEEE QGTLLTEAIKPRKKLPPLLTQLSERPPEKLHYMGVSYGVTLQLYRFWSKLSFLPVYMRLT INETTGENTCVMLRELQNAGLDINAGWLDAFANDFKRRFITLLGFEFRRFPASLALAILK KEHYVELESNPNEEVSNELARKDIKEFFTDYDMKRLHAYAQELVDYHVITDLLPALARFY FLDRFGSAKVAPTQAQILIAMGLQYKNVDETSKEMGIPANQVLALFNKSIRRISRFIRGM QEEEAEEELEGKKEETTENGTLKEEAKEEAKEEGNEEEEDDENKKQVRDSMLESLKLGAY SIKGSTDDWKSALKGKTNLSSITLSTKAKGDAQDNKFAKRPNKGKEKPGKQKRSKH

$>$ A_thaliana

MRKKVDERIRTLIENGVKLRHRSMFVIIGDKSRDQIVNLHHMLSKAVIKCNPSVLWCYKD KLDISSHKQKRSKQLKRLRERGQLDPEKLDAFSRLLDVGRVTHCLYKDSERILGNTFGMC ILQDFEALTPNLLARTIETVEGGGLVVLILRSLTSLTSLCTMVMDVHDRFRTESHSEAAG RFNERFLLSLASCKACVVMDDELNILPLSSHIRSITQVPTEKDSEGLSEAERDLKSLKED LSDDFPVGPLIKKCCTLDQGKAVVTFFDAILDKALRSIVALIASRGRGKSAALGLAVAGA VAAGYSNIYITAPSPDNLKTFFEFVCKGFDALEYKEHLDYDVVKSANPDFKKAVVRINIF KQHRQTIQYIQPHEHEKLSQVELLVIDEAAAIPLPVVKSLLGPYLVFLSSTVSGYEGTGR SLSLKLLQQLDEQSRAPATGLEGSGCLFKKIELTESIRYGSGDPIESWLNGLLCLDVATC LPNPACHPSPSQCDLYYVNRDTLFSYHKDSELFLQRMMALCVSSHYKNSPNDLQLLADAP AHHLFVLLGPVDESQNKIPDILCVIQVCLEGKISENSALQSLRDGHSPYGDQIPWKFCEQ FRDTEFPGFSGARIVRIAVHPNAMKMGYGSAAVELLTRYFEGQIAPISEAEDKVDVEHAP IKVTEAAEKVSMLEEQVKPRTNLPPLLVPLHDRRPEKLHYIGVSFGLTLDLFRFWRKHNF APFYSAVTGEHTCMLLKPLKNDELEVNESDELGFFTPFYKDFKIRFSKLLSDKFKKMDYK LAMSVLNPKINFAEVDSSGSSSGGFLKTLNGILSPYDMERLRAYTENLTDFNLVYDICKT LAHQYFEEKLPVSLSYVQASILLCLGLQETDFSSIERQMQLERGQIHSLLLKVARELYKY LNGVAGKEIKSALPRLKERELTAHNVSVDDDIREGAKQVEEQMKKEKIEGLMDSELQQYV IGDKEAEALQHSKISSSGI ISVKSTKSENENGFDKSTKKRSSDKRSSSSSKSKSSKKRKS LKE

$>0$ sativa

MRKKVDERIRTLIENGVRERQRSMFVIVGDKSRDQIVNLNYMLAKSRVKSRPSVLWCYRD KLEISSHKKKRAKQIKKLMQRGLMDPEKADPFSLFLETSDITYCLYKDSERVLGNTFGMC ILQDFEALTPNLLARTIETVEGGGLIILLLRSLSSLTSLYTMVMDVHERFRTESHTQAAA RFNERFLLSIASCKSCVVMDDELNILPISSHMKFIQPVTNNEDSEGLSERERELKDLKDQ FREDFPVGPLIGKCFTMDQGKAVINFLDSILDKSLRSTVALLAARGRGKSAALGLAIAGA IAAGYSNIFVTAPSPENLKTLFDFVCKGMNALEYKEHLHYDVVKSADPELKKATIQINVY KQHRQTIQYLKPHDHGKLSQVELLVIDEAAAIPLPIVKSLLGPYLVFLSSTVNGYEGTGR SLSLKLLQQLESQSQPSAPNNGPNSSRLFKKIELNESIRYASGDPIESWLNDLLCLDLAN SIPNISRLPHPKECDLYYVNRDTLFSYHKESEIFLQRMMALYVASHYKNSPNDLQLMADA PAHHLFVLLGPVDESKNQLPDILCVVQVCLEGQISRKSAMKSLSEGRSPSGDQIPWKFCE QFQDNVFPSLSGARIVRIAVHPSAVRLGYGSAAVDLLTRYYEGQMTLFAEDEEENEEPEV RITEAAEKASLLEETVKPRANLPPLLVHLRERRPEKLHYLGVSFGLTQELFRFWRKHNFY PFYVGQIPSAVTGEHTCMVLRPLNSDDIEVNESSKCGFLDPFYQDFRQRFRRLLGTSFRH LNFKLAMSVLASKIDFSDHEPSDYYTNITSKILGDMLSPHDMKRLEAYSNNLVDYHLILD LVPILAHQYFSEKLPVTLHGAQAAVLFCMGLQDKDIGATKEELGIEREQVLSNFIKTMKK LYGYLHNIAGKEIEATLPRLKEIDTAPLKSLDEDLDEAAREVKEQRRAIDEDDVDPKFLQ KYAIDADDDEIEKALNGGKISASGVISVKSNKTKADKQEKRKEMKKSKRKGNDGEKSESK KKRS

$>$ A_trichopoda

MRKKVDERIRTLIENGVKSRHRSMCVIVGDKSRDQIVNLYHILAKSVVKSRPKVLWCYKK 
bioRxiv preprint doi: https://doi.org/10.1101/2021.11.30.470322; this version posted November 30, 2021. The copyright holder for this preprint (which was not certified by peer review) is the author/funder. All rights reserved. No reuse allowed without permission.

ELFLSSHKKKRMKQLKKMMQRGLLDPEKDDPFSLFVTSTEIRYCYYSETENILGNTFGMC ILQDFEALTPNLLARTIETVEGGGL IVLLLSSLKSLSSLYTMVMDVHERFRTESHSEATA RFNERFLLSLASCKACVVMDDELNILPVSSHIRSINPVSVKEGSEGLSEGDLDLKNLKEQ LHDDFPVGPLVGKCRTLDQGKAVITFLDAILEKTLRSTVALTAARGRGKSAALGLAIAGA IAAGYSNIFVTAPSPENLKTLFQFVCNGFDALDYKEHLHYDLVQSTNPDFKKAIVRVNIY KQHRQTIQYIQPQDHEKLSQVELLVIDEAAAIPLPVVKSLLGPYLVFLSSTVNGYEGTGR SLSLKLFQQLQEQSQSAVNNESGSVSGRAFRKIELSESIRYSSGDPIEAWLHGLLCLDAT NFIPNITGRLPHPNECELYYVNRDTLFSYHKDSETFLQRMMALYVASHYKNSPNDLQLMA DAPAHHLFVLLGPVDESKNVLPDILCVIQVSLEGQISRASVMKSLSEGYQPSGDQIPWKV SEQFQDKVFPSLSGARIVRIAVHPSVLRAGYGSAAVELLIRYYEGQMADMAEIDSEQVEE KTPVKITEAAEKVSLLEENIKPRANLPPLLMRLDERRSEKLHYISVSFGLTQQLHNFWSK HKFVPFYIGQIPSAITGEHTCMVLRPLNNDEVELTGSGKAGFFDPFYQDFRRRFTRLLGS SFRTLNSALALSILDPKINFGEQAPSEEPSISGILGMKAMLTPYDMERLDAYANNLADYH LILDLVPILARLYSEERIPVSLSYSQAAVLLCMGLQHQDISYLQGQLKQLGKQQILSLFN KVIKKIHKYLHNAAAKEIESTLPRLKEVVMTPHSKSLDEDIDEAAREVIEKMKGESELGG MLKPELLQQYAIVDREADFEDALLKGERKISSSGLLSVKSNRKNVDKKSKLKEKEKGENR GVSAHSSRPHKKSKA

$>$ N_colorata

MRKKVDERIRTLIENGVKKCHRSMFVIVGDKHHYQIVNLHHMMRQATVKPMTSVLWCYKK NLQLSSHQKKRMKQIKKMRQRGLLDVEKEDSYLLFLDSEKIRYCYYSETENILGGTYGMC VLQDFEGLTPNILARTIETVEGGGLVLLLLSSLTSLTSLYTMVMDVHKRFRTEAHPCAAS RFNERFLLSLALCEACIVMDDELNILPISSHMWSITPVPALEGPGGLSESELELKNLKEQ LHDDFPVGPLIGKCRTLDQGKAVISFLDAILEKSLRMTVALTAARGRGKSAALGLAIAGA VAGGYSNIFVTAPSPENLKTLFEFISKGLEALDYKEHTHYELIRNPDYKNATTRVNVFKQ HRQTIQYIQPQDHEKLSQVELLVIDEAAAIPLPVVTSLLGPYMIFLSSTVNGYEGTGRSL SLKLFSKLEAENHLLAKKDDKSLSGSLFRKIELNESIRYAVKDPIESWLHGLLCLDATNQ IPRLNSSGLPPPSECDLYYVNRDTLFSYHKESEIFLQRMMALYVSSHYKNSPNDLQLMAD APAHHLFVMLGKVDESKNQLPDILCVIQVCLEGQISRESVIKSLSHGSQPSGDQIPWKFS EQFQDNAFPSLSGARIVRIAVHPDIVGSRYGSAAVDLVTRYYEGQLTNISEAECDDPKYD EFENASLKVTEAAEKVSLLDESIKRRANLPPLLVHVQERRPEKLHYLGVSFGLTWGLYGF WAKNKFAPFYISQIPNAVTGEHTCMMLRPLNTDDVELNGANEWGFLDPFYQDFRQRFIRL LGSSFRSLHPALALRILEPRVGAKKDIRAEAKEPTSTSNGVLGLEATTLTPYDMKRLESY TDNRMDFHLILDLVPVLSHLFFGSKLPVELDYIHKI ILLCIGLQNQDFTYVKGVRIPLPC REGHRFAKEVQLSGEQITASIFKVMTRIYRYLYGLATKEVESTLPRLREVVMLPDSRSVE DDLNDAAKQVVEKMNKEEQDYLLNPDMLKQYVIVGQENEIEAALLKGQGKISSSGVVSVK SSRRKEENGKVKFKNGKKRENDRGKFNPKKRKV

$>$ S moellendorffii

MRKKVDSRIRTLIENGVKSRHRSLFVVVGDKNRDQVVNLHYMLSKAVVKSRPSVLWCYKK DLFLSSHKKKRMKQIKKMMKRGLLDAEKDDPFSLFVASTSIRYCYYAETEKILGNTFGMC VLQDFEALTPNLLARTIETVEGGGI ILLLLSSLSSLTSLYTMMMDVHSRFRTESHEDVVA RFNERFILSLASCKACIFMDDELNILQVSSHVRSISSLPVLEGEEGLTESEKDLKELKDS LVDTQPAGPLVSKCKTLDQGKAVVTFLDAISEKTLRSTVALTASRGRGKSAALGLAIAGA VAFGYSNIFVTAPSPENLKTLFEFVCKGFDALEYKEHIDYDLVQSTNPAFNKAVVRVNIF RQHRQTIQYIQPQDHAKLAQAELLIIDEAAAIPLPMVKALLGPYLVFMCSTVNGYEGTGR SLSLKLIQQLRSQGKSESAPSGRVFREVELAEPIRYGAGDPIEGWLHELLCLNAADHIPS IEGRLPPPSECRLFYVNRDTLFSYHKASELFLQRMMALYVASHYKNTPNDLQLLSDAPAH HLFVLLGPVDETQNTFPDILCVIQVCLEGKISRKSALKSLSDGSSPSGDQIPWKVSQQFQ DSEFPSLSGARIVRIAVHPNLIKAGYGSAAVNLLVRYYEGQFTDLEEEDDEDDVVHKHPV KVAEAAAEISLLEEQIKPRANLPPLLVGLHERNAEKVHYVGVSYGLTQQLHNFWKKLKFV PVYVSQIPSAITGEHSCVVLRPTEIGEVDTATAEQGSWVRPFNEDFSRRFISLLGSTFRS FSPGLALSVLKPQIDFDEESQETISTEGILGGKNALLTPYDMKRLESYANNLADYHLIID LVPTISRLYFMRTIPVTLSACQAAILLCIGLQQHDISFVESALDLPRNQVLALFNKSVRK LHGQLHKAAAKEIEETLPRLREVVMNPHAVSVDEDLEEAAAKVKADQKEKVSSMLNPESL QHFAIGGNDVDFEAALGGKVPKSGLVSVKSSENKGEAKKRPPQNKQLKNKSSKKSR $>$ P_Patens MRKKVDPRIRMMIENGVKSKQRTMFVVVGDKGRDQVVNLHYMLSKAVVKARPSVLWCYKK DLFLSSHKKKRMHQIKKMMARGLLDPEKDDPFALFMASTNIRYCYYADTHKILGNTFGMC VLQDFEALQPNLLARTIETVEGGGIIILLLSSLTSLSKLYTMTMDVHARFRTESHGDVVG RFNERFILSLAACKSCLVMDDELNPLPLSSHTKSITPLPNLEGEEMLSEADKDLKELKDS LRDTQPAGLLVQKCRTLDQAKAVITFLDAISEKTLRSTVALTAARGRGKSAALGVAIAGA VAFGYSNIFVTAPSPENLKTLFEFIFKGFDAMEYKEHIDYDLVESTNSAFNKAIVRVNIF 
bioRxiv preprint doi: https://doi.org/10.1101/2021.11.30.470322; this version posted November 30, 2021. The copyright holder for this preprint (which was not certified by peer review) is the author/funder. All rights reserved. No reuse allowed without permission.

RQHRQTIQYIQPKDHEKLAQAELLVIDEAAAIPLPIVKALLGPYLVFLCSTVNGYEGTGR

SLSLKLIQQLREQSNKLQSNGSTDGGSVPGRTFRELELAEPIRYKAGDPIEAWLNELLCL DAANHIPNIEGRLPHPQECQLYYVNRDALFSYHKESEMFLQRMMALYVASHYKNTPNDLQ LMSDAPAHHLFVLLGPVDDTQNRMPDILCVLQVCLEGEISKQSALRSLSEGNLPNGDMIP WTISQQFQDPEFPSLSGARIVRIAVHPSLIRAGYGSHAVDILSRYYEGQLSDLTELEDDA AEPKDSQKLVTEAASEVSLMEETVKPREDLPSILTLVSERKPEKLHYLGVSFGITQALFN FWQKRKFVPVYVRQTPNEITGEHTCIVLKPLQSDDVEGVAGVSGGWLSPFNQDFRRRFMS LLGFQFRMFAPALALSILDPKITFTEQETMVGASTENI IRGLTPLLTPYDLKRLESYASN LVDYHLILDLVPTVARLYFLERLPTSISYGQAAILLCLGLQYHQLEHVEAQLKLPGSQIL ALFNKTIRRLYNQLHKAASKDIEAALPRLREVTMMPHETTVDEDL ISGAKKVEADIRSKM EENVLDPEKLQRFAITGADADFDEALGSGKIPKSGYVSVKSSKRPDGKDQQKVGKAEKAK RKKGADFKRPGGKKSKH

$>$ M_polymorpha

MAGLPSQQPQGPAAGRGRGMTVPAWMKVTPAGPSPPAASPTSGPPQAHIPGNTPPSGPLP PPRMPGLGPSGAPPNYGVPQMQFRPQGPPQPGQQFMPSGGQQYRPAGPPPGMGPPQHGMP PNMQGMGPQQGMPPSSQPSQYSPSPMQQMLPRPPQQPQPPQSSQGPPMSYPQQARPMAGG PPPQQQPQPHGMPPPSGPGNPGMQPPSSFGFNSSLPYPQAPNSYGMTPPHYQSPMQPQSI QPMQQSSQPWPMIPGQSMPPALPQGLQAAAQPPPAGASSLPGATALSAQSSVPGPSDWQE HTAPDGRRYYHNKRTKLSSWEKPVELMTPTERADASTVWKEFTTADGRKYYYNKNTKQSK WTMPDEMKLAREQVDKSTATTTSGSSVAEVVPPSVTLGTTPTGTNPVSFLPPAASPKPSV IVNPSPVFLTPPVALPIAPPVTTVPAFTSSTPSGIPPSVAALPAESVKEDSVETSAKEEA EETSAQDLEEAKKAMPLTGKINITPFVEEKPSPVAEEPLTYANKTEAKNAFKELLESVHV ESDWTWDQAMRVIINDKRYGALKTLGERKQAFNEYLAQRKKLETEEKRLKQKKAREDFLA MLEESKELTSSMRWSKALTLFEEDPRLQAVEKDREREELFEDYLVDLERKEREKAREERK KNLAEYRSFLESCDYIKANTQWRKVQDKLEDDERCSRLDKIDRLETFQEYIRDLEKEEEE EKKLQKEQLRRKERKNRDDFRNLMEEHKVAGTLNARTQWRDYLVKIKDRVEYQAVAANTS GSTPKELFEDFAEELDKQYHEDKVRAKEAMKAGKISVTSTWNFDKFKAAIAEAGDLEAIA EPNLKLVFDDLLERAKEKDEKEAKKKRRMLDDLTDVLRTMKQVTASSKWEECRPLLEETP EFRAFTEESSRKKVFEDYVTHLQQRAKEKDRKREKEEKVGPEVAQNGAKKEKEKEKEKER ERRKEKDKDKDKDKDKDKDKDKEREREKDKDKEKSKREKEVVHDVSETGNNHVHKEEKKK DKEREKDRDRERDKDKKYRKRHRSSSEDAGSDREDKDKEDSKRSRRHGIDHKKSSRKV $>$ C_braunii

MRKKVDSRIRMLVENGVKTRQRTMFVIVGDKGRDQVVNLHYMLSKAVVKARPTVLWCYKK ELYLSSHRKKRLRQIRKMMQRGLIDPEKEDPFSLFMASTNIRYSYYSETHKILGNTFGMC VLQDFEALTPNLLARTIETVEGGGAIVLLLSALSSLSRLYTLAMDVHARFRTESHSDVTG RFNERFIMSLASCRTCVVMDDELNILPLSSHIRELQPVHQLEGEEGMSEAERELKELKES LADTQPIGSLVAKCRTLDQAKAVITFLDAASEKTLRSTVALTAARGRGKSAALGVAIAGA IALGYSNIFVTSPSPENLKTLFEFVFKGFDAIEYKEHIDYDLVESTNPAFNKAIVRVNVF RQHRQTIQYVQPQDNQKLSQAELVVIDEAAAIPLPVVKALLGPYLVFLSSTVNGYEGTGR SLSLKLIQQLREQSAKAGDPASATNGGSEAVVAGSGRVFKEVELSEPIRYAQGDPIEAWL HELLCLDAANHIPRISGRLPHPNECELYYVNRDTLFSYHKASEVFLQRMMALYVASHYKN TPNDLLLMSDAPAHHLFVLLGPVDESKNALPDILCVLQVCLEGEISKKSAMKSLAEGNQP TGDLIPWTISQQYQDSEFPSLSGVRIVRIATHPDLVRAGYGSRAIELLARYYEAQMTDLG EQEEDEEEEEKEDLPTDKAKITEKAEEAVSLLTEQVKPRTGLPPLLVSLAERKPEKLHYM GVSFGLTQPLLNFWRKMKHVPVYVRQTPNDITGEYTCIMIRPLQSDELQQSDGEAWGSAM WLSPFLEDFKKRFICLLGVSFQKFPPALALSILDPKIVFSEQESSEGVRAPGVVGSGMAL LSPYDMKRLDSYSRNLVDYHLILDLVPTIAQLYFLEKIPATVSYGQAAILLSIGLQRQEL SEVEKSFATEFPALQQRPSTIRW

$>$ C_reinhardtii

MRKKVDARIRTLIENCAKTRQRALLVLVGDKGRDQVVNLHYMLSKASVKARPSVLWCYKK ELHLSSHKKKRMKQIKKLAQRGLLDPEKEDPFALFVASTNIRYCYYHETQNILGNTYGMA VLQDFEALTPNLLARSIETVEGGGLVVLLLSNLNSLTQLYSMTMDAHSRFRTESHQDVVG RFNERMVLSLASCANCLLLDDELNVLPTSSLIRYIEPLPTNPDGTVVDDPARSARQELGE LSGSLADTQPAGALVGRCRTLDQARAVVTFLDAASEKTLRSTVALTASRGRGKSAALGLA IAGALALGYSNIFVTAPSPENLRTLFEFVFKGLDSLDYKEHIDYDLVESTNPSFGKAVVR VNVFRNHRQTVQYIQPQHHAKLAQAELLVIDEAAAIPLPVVKQLLGPYLVFLCSTVNGYE GTGRSLSLKLIQQLREQGAKISAGDNKAGSKAAGGADGTAAPIANGTSGRTFREVHLGEP IRYAPGDPIESWLHELLCLDAGSHMPKPPPRLPHPSDCELFFVERDTLFSYHKASEKFLQ QMVALFVASHYKNTPNDLLLMSDAPAHQLFVLLAPVDQTANTMPNILAVAQVALEGSISK RSAVNSLAKGELPQGDLIPWTVSQQFQDADFPALSGGRVVRIAVHPELGRAGYGSRVLEQ LRRYYQGELTSLDEEDDAGGRGGAGAGGSGSGEEDGDEARRRSGADAAANADGDAPLLSE 
bioRxiv preprint doi: https://doi.org/10.1101/2021.11.30.470322; this version posted November 30, 2021. The copyright holder for this preprint (which was not certified by peer review) is the author/funder. All rights reserved. No reuse allowed without permission.

RLAPRKGLPPLLVPLSDRKPDRLHYLGVSYGLTQQLFTFWRKAGYQPLYLRQSASDTTGE HTCVMVRPLEHPEVEGTAWLSPFVSDFKGRFTSLLGGAFREMAPALALSILDPQLNWGEE EAQRATQAGVVVHRGDGSGLDPYDLKRLQAYCSSLVDYHLVLDLVPPLAAAYLRGRVPVS LSYGQAAIMLVLGLQLREVSALEESLGLPSNQVLALFNKAMRKV

$>$ G_Sulphuraria

MKKKIDARLKTLLEVSCQNNHRALLVIIGDRGKNQVVNLHYMLSKISNIPKPSVLWCYKK ELGFSSHRKKRMKQIKKLAQRGLLDTSRDDPFEIFVSNTHIRYCYYAETHKILGNTFGMC VLQDFEALTPNILARTVETVQGGGLVVLLLGTIQSLRQLYTMAMDVHDRFRTQAHKDVVA RFNERFMLSLSRCQSCLIVDDELNVLPLSSPMKEGTFALSDKQKEQIKKQQQEWSELKSN LEETPILGELISRTKTLDQAKALLTFIEAITEKTLRSTIALTAARGRGKSAALGLAVASA IGHQYSNIFVTCPSPENLKSFFDFVLIGFDALQFKEHVDYEVLQSTDASLQHCVVRINVF RSHRQTIQYIDPRDASKALHQAELLVIDEAAAIPLPIVKSMLGPYLIFMSSTITGYEGTG RSLSLKLFDQLRKGAHSDNRPDSFHLAEGGRTFREITLEAPIRYAIGDPIESWLYDVLCL EAGKGPQPLIYGCPHPHDCQLYRVNRDALFSGHPLAESFLQTIMALFVSSHYKNSPNDLQ LLSDAPAHRLFVLIAPTKKQLDSNGLPQVLCAIQVCLEGQISKEYAQASMSRGYRGAGDL IPWTISQQFQDSSFASLSGVRVVRIATHPDVQRMGYGSRALTLLNDYYSGKMISLTEDGW RGTLSDEPSTITNVDNSNDTLNHERLEPRKELPPLLEPLNVPDRVDYLGVSYGLTYELFC FWKKQGMVPLYIRLVANEYTGEHTCIMLKQLPEQSKMSWLSNFFHDFIRRFLALLPFAWS SWNPALTLQIIDYGKEYYASHQVTMLNEHNMHLLLSTFDIQRLEAYAKNLIDYHMIVDLL PTIAKLYFSGQLDKEIHLSAAQSAILVSVGLQYMDLDRLEKVLNVPAQQLLALLNKMIRK FAHYFKELEWSLMESHDASTIITETKTSMKDTNETLEPKQAPLVKKPKKNMEHKDKKEHR KHRLE

$>$ C_crispus

MKKKVDPRIRQCLQEGVNNNHRSLIVLVGDKGRDQVVNLHYMLSKMTLQSRPSVLWCYKH ELGFSSNKKKRMKIIKKRIKQGLVDPNRDDPFELFISSTSIRFAYYSESHKILGNTYGMC VLQDFEALTPNLLARTLETVQGGGLAVILINNMSSLKKLYSITMDVHARLRTEAHRKVVP RFNERFILSLADCPAAIVADDELNVLPISSHVRAMSGEVTSTANRPTTENEPELKELKES LADTPPIGPLIAKAKTLDQAKALLRFEEAISEKTLRSTVALTASRGRGKSAALGLAVAAA VAHDYANIFVTSPSPENLRTFFAFLFVGFDAMDYQEHTDYEIIQSTDSGLNNAIVRVNVF RSHRQTIQYIDPIDAPRSLSQAELLVIDEAAAIPLPVIESMLGPYLVFMCSTITGYEGTG RSLSLKLMTKLREGSARVPSQYKGVNGNSNRQSDRDAAAVSSRTGSGRVFREVQMEIPIR YGQKDPVEAWLYDLLCLEAGGVRQVLTGGAPHPEECELYYVERDALFSRHSASEAFLHRI MALFVSSHYKNSPNDLQMLSDAPAHGLFVLLAPTRPDTERLPDVLCAIQVCLEGKISSKS SKTQLSRGQRASGDLIPWTISQQFQEPDFATLSGARVVRVATHPDVQKMGYGTRALKLLL EYYKGEHNISLENNAVERLSEQNPPAGQENGYTDSEQGDADQQKKTLLNEIIGPRKNIPP LLRRLHERSAENLDYLGVSYGVTQGLYSFWSRLGFMPLYLRLTPNDLTGEHTCIMATTSV CSSEETKPSDWLSKFNQDFRRRFVHLLGYDFRSLPPGLALAILNTGAEFRESGDAEERSS IDEIKGLFTPYDRRRLESYSRSLIDYHVILDMIPKLAELYGTGSLGLRDGLRLSPAQNAV LMGIGLQRCSVDDLEKRLGIQASQVLALLNKVIRKMSTFLRDMEETDIASRIGQTQGARG MIPGVTIELPNSNKAEFAALSTDDPSDEKGTPGKSLKAEEDGAGATEKGTRMSNFKVGGS KDEWKAALPKNALADGIPSTVSIKSGKKKSKRKTISESKDVGTPRKRKKKQVK

$>$ E_siliculosus

MVRKKVDARVRSLIENGVKSNHRSTFVLVGDHGKDQVVNLHYILSKARVASRPSVLWCYK KELGFSTHRKKRMRQLKRQKKSGLASAGGASEDPFELFVNSTNIRWCYYKDTERVLGQTF GVLVLQDFEALTPNLLARTIETVEGGGLVILLLKTVTSLKQLYAMSMDVHSRFRTESSGD VVGRFNERFMLTLGDCKSCLVCDDELNVLPLSSHARDIVPLPPVDEDAETTEAKELRELK ESLEGTPPVGPLVAGAKTLDQARALLTFMEAISEKTLRSTVALTASRGRGKSAAIGLCLA GAIAYGYSNIFVTAPSPENLSTVFEFVLKGFDLLHFSEHLDYEVIQSTNPAFNRAVVRIN VFKDHRQTIQYIQPHDSEKLSHCELLAVDEAAAIPLPLVKSLLGPYLVFLSSTVNGYEGT GRALSLKLIDQLRKQQGTAAAQAARIAASEIHGTGRKKGKEKVHENRWKVAADAAANASK HGGGGGGRTLREINLETPIRYGANDPVEAWLYRLLCLDAKVGHHRLVCGTPAPKDCELYY VNKDALFSYHTLSEGFLQRVMSLYTSAHYKNQPNDLQMLSDAPAHHLFVLLGPKAMEQGA KGNLPDVLVVVQVALEGQISRASLEASLSRGHRASGDLIPWSLSQQYQDGQFAGLSGARI VRIATHTDVQKMGYGSRAIDLLTKYYEGGLFSGTGGEEGSEEGSEESSEEESEEEEEDDD DDDDESSDEEEEGNGTANKKKKKASAKGGGLSSERLAPRKKLPPLLVSAGERPPERLHWL GSSFGVTGQLLRLWKRAGFRLVYLRQTTSDLTGEHTGVMLRALDTEGMKEKPEDGWLASF AADCRCRIVSLLGHEFQGFDCTTALSLVEAAPGGAAVEGGEAPAAGGARGAVGARELGYY CGPHDLKRLGLYSRNLVDYHLITDLLPMVSKLYFLGRMPSTRMSHLQEAILLAMGLQHRS VTALSKELQLPDNQVLALFNKAVRKLCNCLTEVAEKEAEGEMETHLAKKKRLQTVKSAGE MSMLPASMDEDLEGGAKEAMKKMEGKQFAVMLY

$>$ P_tricornutum 
bioRxiv preprint doi: https://doi.org/10.1101/2021.11.30.470322; this version posted November 30, 2021. The copyright holder for this preprint (which was not certified by peer review) is the author/funder. All rights reserved. No reuse allowed without permission.

MVRKRLDDRVRALLERSVVTGQRSMLVLVGDHGKDQVPNLHQILTKCSVQARPKVLWCYK KELGFSTHRKKRMKKLKRDKSRGLVGGEADQADNFELFVSQTDITWCYYKDSHRVLGTTV GVLVLQDFEALTPNLMARTIETVAGGGLVIFLLRTVKSLKQLYAMSMDVHARYRTESAGD LVPRFNERFILSLGKCPNCLVCDDELNVLPVSRKALNDLSPNAGWSKGDAGEVIVQDTPE QRDLKEIQEALLDTPHVGVLVELTKTLDQAKALLVFLEACSEKTLKSTVAMTAARGRGKS AAMGLCLAGAISLGYSTICVTAPEPENLVSVFDFLCRGLKALKYQEHMDYSVTYNSASGR EQTKCITAINVHRSHRQVIQYVDPAETDKFTSAEIVAIDEAAAIPLPVVRALMSHPDRLT FLSSTINGYEGTGRALRGRHAEMQAASSAANSIVGAKSKKGEAKVHEQRWAAAAAAILEA SEEIELLTPIRYAHGDSVEAWLNKLLCLDCGSASNLKLNGGAPAPGDCELYSVDRDALFS FHKLSEAFLQKVMGLYTSAHYKNSPNDLQMLSDAPAHSLFVLLSPSAEQDANSLPDVLTV VQVALEGRISRKAVEAQLARGHRSAGDLIPWTISQQFGDSKFAQLSGARIVRVAVHPSVQ GMGYGSRAIELLYRFYNEEMVSLVNDEGNDDADSDAERNGEEESDNDEPTTSGIGILGEN LRPRKELPPLLLPLTEVDMPRLDWVGTSFGLTLQLHKFWSRSGMRMLYLRQTKNELTGEH SSIMVRALPRRSGVDDSWLYAYLSDARRRFTTLFSGPFRHLDVRLALSVFDNMDVPSNTT EAKQRAGALAGTLTFKELDYFLTPYDLKRLELYGRNLCDHHLVMDLLPIIGRLYFTGRFG SDFNLSSVQAALFCGIGLQNKSVDILTRELGLPTNQVLAMFNKAVRKMSIALNSVVEEKE KESLLTGEKRSRIEESAEQMRHVSRQTLDEDAEQAGQEAIATLRANEMANHLPELAHDTE MLKYVVKGSDKQWEKVLQDKDKREKLLTMTT

$>$ T_thermophila

MRKKKVDQRIQILMENCIKLRQRGFFIIVGDRGKEQVVNIHYLLNKMNFKSKPNVLWCYKK ELGFSTNKQKRMKEIKKLQKKGLYDNNVDDPFQLFVTSTQIRYCYYKETHKILGQTYQML ILQDFEAITPNVLCRTIETVEGGGFVIMLFKTMSSLKQLYTLTMDVHNRYRTDQFQDVEP RFNERFILSLSKSENTIVMDDELNILPISNLIENIKSVSADDSLDSKMTKELEELKESLK QNVLVGPFINIAKTLDQARAIMTFIDAITEKNTRATISLTAGRGRGKSAAVGVAIAAAIV CGFSNIYVTAPSPENLKTLFEFIFNGLEQNNYKENMHYDIIQSTNPDFNNAVIRVNVFKT HKQIIQYVSPTDFASIGHADLVVIDEAAAIPLVYVKQFLGPHLVFLCSTVNGYEGTGRSL SLKLIQQLKSQSKMNSSSNSEEKQTAVSSSGSRILRELTLNDPIRYSPNDPIERWLNDLL CLEASQAVPLKSGLPHPNDCELFMVNRDTLFSYKKSTEQFLFNLMSLFISSHYKNSPNDL QLLSDAPSHAVFVLMGKIKEGEKKSEMPDILCAVQVALEGKISKETFNQQNENRVRPAGD LIPWI ISEQFQDREFPSLSGVRVVRIATHPAAQKMGYGSKSLELLQKFFEGELIDLSNET LNRENYFLKAQRTADGEKKLKPLLKRLTEIEPPQLHYLGVSFGLSKELFKFWKKSGYYPL YLRQAKNDITAEHSCIMLKSLKKQNDMENENVQEENKYNDQWLKYYSDDFKKRFLQLLSY EFKDVDVQLSLGVVDPTFVTDDNDSRLTNDLGLGDKDKNQLGNYIMAPISNKEQLENYIN TYDLKRLEAYSRNLADFYLIIDLLPEISKMLFLNRLQSIKMSYSQCAIFLGLGLQHKTIE KLARELGIPVSQVMAIFNKGIRKVTQLFRSLFEQEIMKELNFGNSKEALEKMNPIKDNEN EIQDAGKEYLKSLLEKDNKKDEKEDKKSDKLNKKRHARYNFGTEDIDVGNASINQNTGTI TIARDSKVALNQDFTNYKSASAAQAAAVKNAKKKLKKN

$>$ P_tetraurelia

MKKKLDSQIQALVQSGINEHIRSIFVMIGDRSKYQVATMHQLLSKLSMIQKPKVLWCYKK DLGFTTHKKKRETEIKNQQMKEETQLNEPFNLFLSSNEIRYCYYAETQKVLGNTYNMLIL QDFEALTPNILCRTVETIAGGGIILFLIKTMSSLKQLYSLTMDIHSRLRTPSFKEVQPRF NERFIISLSRCQRCLFVDDELNLLPVSSTALEKIEQGTPINSNQVADFAQTVPNEIAQKL IKNCKTLDQAKIILGLIDSLQEKVFRYTISISAARGRGKSAAIGMAAAAALKLGFSNIFV TAPSPENLVTFFEFLISSLETLDFRQNQHFDVIQSTNPDFNNAVIRVNAYKTHRQFVQYI QPQDFVKVANNADLVFCDEAAAIPIQFVKSMFGSHTLVLSSTINGYEGTGRSLSLKLLQK LRETKQTGQARLLKELQMNDPIRYSNKDIVEKWLYELLLLDASDAPKLQKGLPHPNDCEL YLCNRDTLFSSQKTSEKFLFNLMSLFISSHYKNSPNDLQLLSDAPAHAVFVLLGPLPTDK TMPDILCAVQVCFEGAIPKDLVDQVTNQQKPSGDLIPWTLSKYYLDNQFPQYSGVRVIRI ATHPNAQKMGYGSKAMELLNRFCKGEILDPSAQLIHIDFREQATAEDEKDQLIQPKKKLP PLLKKLSEVYPPQIDYLGVSFGLTQELYKFWSKNQFSPAYLSLRKNDTTGEYTCIVLRPV KDEGSGIDNLCKAFSNDFRNRFINLLGYDFKDLEPYLCIDILKANVTTANVDEDIEIAKD DNI ITKEELRQQLSLSDYARLENYCKSMADFYLILDILPSICKIFFAQKCGKAIRMSRTQ AALLLGMGLQYKNIELASEQVDNAVNQSLPLFNKAMRKMRNFIKMVLDDEQHQRTKPKQQ QQQPSKQSVIQQKRGKVNYQIDGQIDQEEIQQSLQTGDVVTLGRSKDQAYINYKKKVKLG $>$ P_falciparum

MKKKKVDNRIKILIENNIAVGQRSMFFVVGDEGKNVVVNFYFLLNRLINRTHNILWCYKKK LDFSTSKKKRYREMKKKIKKGTFDQTIDNNFDMFLKNADIRFCFYKESKKVLGKTYSICI LQDFSYITPNILCRCLETVIGGGLI IFLINKQNELKDLYNLTLNYHKKYTMNGICNVYNN YIHRFFLSLNKCENAMFIDDEMNILPLNENYLKIKKITSKGSDGNGIGNNVTKLKHMEVQ KEGEQSGSNINNNDDDDNDDNDNYSNSNNYSNSNSNNYSHTKSATLGGFLCPDKNLLQEK LKFLESICEENEKKKEEEKIFLYSSKFSRNDTCTKINKNGDIHSSDNKYTRDTYETYDSV 
bioRxiv preprint doi: https://doi.org/10.1101/2021.11.30.470322; this version posted November 30, 2021. The copyright holder for this preprint (which was not certified by peer review) is the author/funder. All rights reserved. No reuse allowed without permission.

TNTSTTANTYNNYISKKTKKKDVYSFLDRNIMNLLNICLSLDQLEVLLNMCKILRNDEEK KKNIKEVLFNLLANRGRGKSATLGLLLSLSIYFNYSNI I ICSGNNEGVQTIYDFLDKGLH ILGFNEFKDYEKIYDMGKIKEIVIFKNMKYLKQRIRFFDIIEEDILNSELMIIDEAACIP IDILKKKIKGEITILSTTLNGYEGTGKTFTFKLLKQLKKKFITQLSYEEFKNMNFLYFDK AYIDLTLTNPIRYSYNDQVEKWLNDFLCLNCNEPTNIKDNICSPSNCELYFVNKNIFKNY NRTSEILLKKIMTLFITSHYKNTPNDIIMILDSQQHHLFILINKNNNHYNNNNILEDQID ELDIYGVLHCAIDGIINNGNVKKTVKLEDI IHLVKNNNTQKENPTLDTKEINDSNDQKLE KKENINQINSDNHKELLINEYEGNLIPYIISDYFNYYFYNYIGIRIVRISIHPSMQNLNY GSEFLKKVCNYYNEYNSKQRHDNNTKQKIKINNNDSNNCGDSNNCGDSNNCDDSNNCDDN VKYSENI ILFNCNGKNIYFDKKLKQIDYIGTCFGLTKGLLIFWQKNKFIPVFLKQQKNEI TGEFSVLMLKDLNEDLKHIIANFYTDFVRSVTKLLPYSFKYMESFIVFNLLHNNNYSIPL KQDDAPTIVKDHNNTDHTYNHNGHIKNNLHNNHFYYDNYEDQHLSFYNNNLINQQTIFYF FHTNDICRLKRFVLEGRNFYEILYLLSTIANLILFKKVKIDLSFLEYTILYAVAFQKKTP KEISDEIKINVNQTNAIFRKI IHRFYNYIKDIMEKQIEKEVNAELNEKLKVKKKRSLNIE LPSEEYKDELERNSLIVKKKLKKEKLSLMKEFNLEGDIKRKEINIKTNDNLEIKKKKKKK KKKKKQETN

$>T$ _gondii

MRKRVDPRVRTLVENCIQLGQRSFFVIIGDRGSEQVVNLHYLLHRYFPAQKPSVLWCYKK DLGFSSHRRKRAKQVKKKIQRGLYDPNIDDPFELFMSSTNVRFCYYKDSHTVLGMTTGMC VLQDFEAITPNILCRTVETVQGGGIICLLLRSFASLQQLYDLSMDIHGRFKTERFSQVKP RFNERFILSLARCRNCLMVDDELNVLPLTSFSQTLRSLKPGMQSEAAEGASAGLPPGLPP DAAEALAEKKKRTEELEALKRDFQETPPAGPLVNLCLSVDQAKAVLAFVDCLAAQASGKK DAANCAAADTRGNGLSALTHLGQVAHQTFALTAARGRGKSAALGLAVAAAVAYDYTNIFV SAPSPENVKTLFEFVVKGLTALGLKEHLDFSVSREDPGLAAAAHASHSSGVSSSENNSSR GGASRLITGVEVHRSHKQKVTFVSPHEREGLGHADLLVIDEAASIPLPIVKSLFGPYVVL LSSTIHGYEGTGRALSLKLIQDLKQNKLSSLSNNPKGGFLARTLKQLTLTTPIRYSVGDG VEAWLHDLLCLDATDPPKLSDAALPPASKCDLYLVDRDALFSFHEASEAFLHNLLSLFVS SHYKNSPNDLMLMADAPAHLLFALLPPVDERTATVPDIYCAVQVSIEGHLSKQAIQQALG RGLRPSGDLIPWTLAQQFADEDFGALTGARIVRIACHPSLQRLGYGTAAMQQLIDFFEMK GVSLKTEGKNALSNSLLSSSQGGDEEDDEDEEDDEDEEDEEDGEGGTPAKGKKKGDKQAE EEQVRDASSAAASASHASSPEAAKVWTKKAFKPRPAPPLLSPCRSTRPDFSIDYFGVAFG LSSSLFRFWTRRNFVPVYVRQTVTDVTGEFSCILLRPAEVEGSLWPGESDKTGAADDRGG DEDLRRKNRWQGIETGRAANELVGRRGGIWLSAFASDFRARFLQLLGGAFRRLPASLALC LVTCSSKPRDNSEAPGSKETSIFPPLSLANLPNFFSSHDLSRLRKYAQQLADLPLILDLL PTLAALFFNDRFPPLAASSVTLSHLQQVVLLAVGLQRKTPDDVSTEFNIPPNQTLALLNK TVHKFVGFFQKLQEAQVEAEVDSQWGVRSAASRRLVVEGGGALLGELPSQSFTEELADEA KKVKRQQKVELKNLLEALGGEEAMKEHSMDAYTEEDFNEAVGAARPGSLQASTASRVFSL KRKNPNKGQKNGGTGEKTGDGGEKKKQKRDFGSFNKNKNKHKVYANR

$>G$ _intestinalis

MAQSVRVNSRGESLKAIDKRLTTLLNSGYESFQRSLIIIVGDGGLYQVPNLFSILKMTRT SQLSIMWCYDEMPKLSSNKRKRNKEFRQLKNRGELQEEELSVEAQFYANTNIRYLHYRDT ELALGTTVDMLILQDFKALTPNIIGQTVECVRGGGLILFLLNNMHDLSQLHAVVMNFHSR FSNINNREVSNDLHIKARFNSRFIALLEYLPRFLLVNDTLEVIQITEGIQNLMTNLSAQD GSVEKRVAAFTAYKNEHKSSSLLAKTFISLCATLEQAKLVIQVLEMLDNVDRIRKLADKN AMLEKEPVERDGKGPREPSRKRYFVREKETYTREDIAESSVDQDVDASLEEQNAGHELLQ TLTQNANALATATRFPICSLIAARGRGKSASLGLIVALMIFQGYNHVLISAPNP INVQTV FEFCIIGLKALDYQEARDYTVRRAGPKATDPIIELRITRKVKQVIAYIMPSTLATYCQAN ERASSSILNFIDAILLDECAAIPLKLVRQIVDNVEANMLCFISSTCYGYEASGRALSLKL LHDLERSGSKRGQGLVKLSLNEPIRYAPNDSIEKWVNQLLCLDQVNGLVTSLEEGPEIAA SFPQPQSCTLYRVNKDALFSGLPQSELFLQTIWSIFSASHYKNSPNDLILLSDNPLHEIF VLCPPITDSKRIPTVLCACQICYEGKLKQNIVKEALSQQKRLDGDMIAWLLSQQFQDVSF SSLSGARVVRIATNASYMRGGYGSHTLELLSSFFKGELLNPTEIKRLEAKKKERIEKLAK QKGISINDAKQEIEALTPALLQNCGTVYPSDLTWLGVGFGITLDLLNFWRKNQYLPVYIR QTRNETTGEHTCLMVRSLKENPFINNFATSFRSRFLRLLRYDFAELPIEIAFKVSWPTNT VALAHDADQVAIRAALTKQDVVRLRGFVRHQLDFMAVRDLVPILAELIFTEKLSPVSLSH ASARIFLGIGLMNMRQIDVAQQLGLVSDVAFSQVESIFTKTVEVLLRILEPLVGLGTELG AGASK

$>$ T_vaginalis

MATTRIKKQIPKNLLDLLSAGISSNHRSFLIIVGPHGTDRIPTINTLLQKKMSTNSKVLWC YHKELHISSNREKRNRQLKQKTQNGTLNEDLATELDLFFANSKQVTFAKYSESHRVLGQT FNILVLQDFSSLTPNILARTVETVSGGGLIVLLLDDMQSLNDLYTITMDYQRGMNRDAFG 
bioRxiv preprint doi: https://doi.org/10.1101/2021.11.30.470322; this version posted November 30, 2021. The copyright holder for this preprint (which was not certified by peer review) is the author/funder. All rights reserved. No reuse allowed without permission.

KVASRFNARFHNSLKSCKNCIAMTDEFDILDSVTPNFEPDSTISEDADLKEILAQYSKDI

ESVNERLEEITDEKQKKQVEHERDEKQQILSIVEKALTADQAKTVGKVIDSIKDRQLGKV IGITAARGRGKSAAIGLSLAAALALGYSNIFVTAPSIANLTALFEFVIVGLTALGFREGE SEDFEVVKSNPTSSKERPYVTRVNVHLSHGRRQTVQYVRPNMTQYLGQCEILAIDEAAAI PLPIVRQLMGPYTTLFSSTVDGYEGTGRALSLKLFKEFQRTMKSRFTQTTLTKPIRYAEG DPIEAWLHNLLLLDVHPQECSLIPALSDTELFLINRDALFNGSRGSEELLRALVSLSVES HYKNTPNDLTLMADSPNHRIFALINMKTVTGKGIPEIIAFVQVALEGKIGDEQFSNAENG KGRRDGDLIPWNIARAFEEPEFAQLTGVRIVRIAVNPEMQKKGYGSVAIEKLVQYYSGHE VVDTKIKPNAKTLMYPLTAIAPEPVDYMGVSFGLTRDLAHFWRKGGFVPLYVSRETNEVT GEHSVIVIRPIGETEWLPKFTTEFKSNVYRLLPTIFRDVTPFVIEEIMFPPPPSEKDSDG RARLTDIDVKKLSRWSGDGIGAVKHLLESVCDFIFVDNPPIKLTRTEMFALLIMGKQMAD EEECAKRLEIQVNQVVALLKSAIGKISRYLNKQRGETDSSMNSKIIEKD

$>$ T_brucei

MPPPTSVASTARSSSGATIKRKVDDRIRKVIENAVHKRHRALLLLVGDRGKDQIVNLHQM VSHANHNAKVNVLWCMKKEPDFGSTSLKQQEKKARLEVKGGMSTDATKEAFQTFLSQTII RFCQYKETHKILGQTFGMAVLQDFEAMTPNTLARTIETVKGGGIIVIMFRAMKSLKQLYT IAMDVHSRYRTEALQDVVPRFNERFLLSLVDCDTSLCVDDDLNVLPITAKMCAVESARTT SYDETLVLQGRQKHEADLAALKERLRASNEVGPLISLCQTLDQGKTILSLMQTVVEKSLN TTCVVTAGRGRGKSAALGLATAGAVAQGYSNIFCTAPSPENLQTFFEFVVRGLQELGYKE RTDFEAMQSTNVEFSKCIIRINVFREHRQTVQYISPNDASKFAQAELAIIDEAAALPLPI VKQMLGPYLVFLSSTISGYEGTGRSLSMKLVADMRKHSGSTNASVAGDDRRLLRELSMSD PIRYGPNDPVEFWLNKLLCLDATVRPIAVKSSPHPNSCELYYVNRDALFSYHPLAEQLLQ AIVAMLVAAHYKNQPNDLQLMSDAPGHHLFILCPSTVDAKSGIPDVFCVIHACEEGHVSS EAIKSNLSRGLRPSGDLIPYTLSQYYLEEGFAKLAGLRIVRIATNPELQRAGYGSRALEL LQQYYTGSVSLRPAASAAATENNTELKAVDDADEVSSTISHTIKPRKRIPSLLVPLIERP YEEIDYLGVSFGVTTPLFNFWKRGGFEPLYLRHAANELTGEHSCVMVRSFGFDLGLLRAE FRRRFIPLLAMPFRQLPTELALSILKDVDVNDPQKLAAATDLSHVAEHNVRVAGQPQCTW KELQEIFSISDLKRLKLNATTFVEGGHVLDLVPALAKLYFESRLFHLPDGAEGVVLSHAQ AAVLLGVGLQCQTIEEVGQQAVFSGVPTQQLRAFLQKAISRIVDHFTRLQKLKLTGSKEE ETVKRNGGNDDADENTHEIYDKEGRVVGLSVEKRVRSVINVDSTLLRDAKSATVGARNDA GGTTGLQRAFKRSKKTRRG

$>$ L_Major

MVKRKVDDRIKTLIDDIARHKHRGLILLVGDRAKDQVVNLHLMISRANHNAKVNVLWCMR EDPDFGSTGKKQQEKRARLEVKGGLSTEASKEAFQTFLAQTNIRFCKYKETHKILGQTFG MAVLQDFEAINPNTLARTMETVKGGGLIVITFRAMRSLRQLYTIAMDVHARYRTETQRDV VPRFNERFLLSLADCDTAMCVDDDLNVLPITHKMKSYGKSCKSDAYNADLAVQGRLQHEV DLANVKETLRPSEDVGPLMQLCQTMDQAKTVLSLMQTVMEKRLDSTCVVTAGRGRGKSAA LGMTIAGAIAQGYSNIMCTAPTPENVQTLFEFAIRGLKELGYRERTDFEALQGVSEEFAK CFIRINVFRGHRQTVQFVSATDTAKFAQAELCVIDEAAALPLTLVKRILGPYLVILSSTV SGYEGTGRSLSMKLVADMRRGSSSGAADARHLKELSMSDPIRYGPGDPVEKWLNKLLSLD ATMENTPLTTSPHPSACELFYVNRDALFSYHPLAEELLQRIQSLLVAAHYKNQPNDLQLL SDAPGHHLFVLCADSVESSAASAPTSSTSGAARQQVPDIFCVIHACEEGQVSAQSIKSHL SHGLRPSGDLIPYTLSQYYLEEGFAKLAGLRIVRIATNPALPRAGYGSRALSLLHQYYSG SISLTAAEPKTAIATHRRGDSEADAREQAGKEHEGDDDESSAPTAMLAPRSHITNLLTPL VDRPYELVDYLGVSFGLTTELLNFWKKASYIPLYVRQAPNELTGEHSCVMVRPMGFDLRP LQREFQHRLLPLLAMPFRHLPTELALSLVGDFEAHDPHKLSAATDLSEVDHHVVRVDGVV QATTDDIAATFTAGDVQRLRLVSTTFIEGGNVLDLVPTLAKLYFGKRLFRCPDGTDGVVL SHAQAAVLLAVGLQCQTTEELAAQAAFTGVSTQQLRGLFLKALSRLSEHLVALQKIPQRA QRDGHNAGVVAGKRARSLSDGAVGDSAADEDVEDAEEVYDSHGNLKGLKVARKVKVQVC 
bioRxiv preprint doi: https://doi.org/10.1101/2021.11.30.470322; this version posted November 30, 2021. The copyright holder for this preprint (which was not certified by peer review) is the author/funder. All rights reserved. No reuse allowed without permission.

Supplementary data $\mathbf{S 3}$

\begin{tabular}{|c|c|c|c|}
\hline Figure 2A & sgRNA_U13_5-Fwd & CACCGAGGGAGGTGGGTCGTCGGGT & sgRNA_1 \\
\hline & sgRNA_U13_5-Rev & AAACACCCGACGACCCACCTCCCTC & sgRNA_1 \\
\hline & sgRNA_U13_7-Fwd & CACCGCCCGTCTGACCTGAACTTCA & sgRNA_2 \\
\hline & sgRNA_U13_7-Rev & AAACTGAAGTTCAGGTCAGACGGGC & sgRNA_2 \\
\hline & Del_U13_UP1 & GGATGTATCGCATTACGGTAGTCACC & Genotyping \\
\hline & Del_U13_Down1 & GCAAACACCACTGGTTCCTACACC & Genotyping \\
\hline \multirow[t]{2}{*}{ Fig 2B } & SNORD13-Rev & GGTCAGACGGGTAATGTGC & Northern \\
\hline & SNORD3-Rev & GCCGGCTTCACGCTCA & Northern \\
\hline Fig2C & & $\begin{array}{l}\text { TAATGATCCTTCCGCAGGTTCACCTACGGAAACCTTGTTACGACTTTTA } \\
\text { C }\end{array}$ & $\begin{array}{l}\text { Mung bean } \\
\text { nuclease } \\
\text { protection } \\
\text { assay }\end{array}$ \\
\hline \multirow[t]{2}{*}{$\begin{array}{l}\text { Fig 2D, } \\
\text { Fig3C, } \\
\text { Fig5G, } \\
\text { Fig6C; Supp } \\
\text { Fig5 } \\
\end{array}$} & h45 rev primer & TAATGATCCTTCCGCAGGTTCACCTAC & $\begin{array}{l}\text { Targeted ac4C } \\
\text { sequencing }\end{array}$ \\
\hline & h45 fwd primer & TGTACACACCGCCCGTCGC & $\begin{array}{l}\text { Targeted ac4C } \\
\text { sequencing }\end{array}$ \\
\hline \multirow[t]{9}{*}{ Fig 3A } & sgRNA-zU13-6F & CACCGAATGTAACCACTGCGCAGAA & sgRNA_1 \\
\hline & sgRNA-zU13-6R & AAACTTCTGCGCAGTGGTTACATTC & sgRNA_1 \\
\hline & sgRNA-T7zU13-6F & GCGTAATACGACTCACTATAGAATGTAACCACTGCGCAGAA & $\begin{array}{l}\text { In vitro } \\
\text { transcription }\end{array}$ \\
\hline & sgRNA-zU13-7F & CACCGGTAGGACGAGAAATAATAAG & sgRNA_2 \\
\hline & sgRNA-zU13-7R & AAACCTTATTATTTCTCGTCCTACC & sgRNA_2 \\
\hline & sgRNA-T7zU13-7F & GCGTAATACGACTCACTATAGGTAGGACGAGAAATAATAAG & $\begin{array}{l}\text { In vitro } \\
\text { transcription }\end{array}$ \\
\hline & sgRNA-T7_Rev & AAAAGCACCGACTCGGTGCC & $\begin{array}{l}\text { In vitro } \\
\text { transcription }\end{array}$ \\
\hline & Del zU13-up2 & CATTTTATCTATGAAAGAAAATCG & Genotyping \\
\hline & Del zU13-down2 & GCCACAGAACTCAGACATAG & Genotyping \\
\hline \multirow[t]{2}{*}{ Fig 3B } & zSNORD118-Rev & GTCAGACAGGATTCATAAAGGTG & Northern \\
\hline & zSNORD13-Rev & CAGACGGTCAATGTGCCACG & Northern \\
\hline \multirow[t]{4}{*}{$\begin{array}{l}\text { Fig 5D; } \\
\text { Fig5F }\end{array}$} & O_NB_CD1-droso & GTCGTAACATATTGGTTGGG & $\begin{array}{l}\text { Northern, } \\
\text { primer } \\
\text { extension }\end{array}$ \\
\hline & ORT_Lh3p & TCAGAGGAGTTTAACCTGCTG & Northern \\
\hline & ORT_Lhumile & CGAGTTAAACCCGTTCATC & Northern \\
\hline & ORT_Amellifera & GAGAACACGATATTCATC & Northern \\
\hline \multirow[t]{2}{*}{ Fig 6C } & C.elegans h34 fwd & CACCATTAGGACTGACAGATTG & $\begin{array}{l}\text { Targeted ac4C } \\
\text { sequencing }\end{array}$ \\
\hline & C.elegans h34 rev & CTAGAGTCTCGCTCGTTATCG & $\begin{array}{l}\text { Targeted ac } 4 \mathrm{C} \\
\text { sequencing }\end{array}$ \\
\hline \multirow[t]{2}{*}{$\begin{array}{l}\text { Sup Fig } 2 \\
\text { A/B }\end{array}$} & 5'ITS1-hs & CCTCGCCCTCCGGGCTCCGTTAATGATC & $\begin{array}{l}\text { probe1; } \\
\text { Norhern }\end{array}$ \\
\hline & ITS2b & CTGCGAGGGAACCCCCAGCCGCGCA & $\begin{array}{l}\text { probe } 2 \text { (mix } \\
\text { of ITS2b + } \\
\text { ITS2d/e); } \\
\text { Northern }\end{array}$ \\
\hline
\end{tabular}


bioRxiv preprint doi: https://doi.org/10.1101/2021.11.30.470322; this version posted November 30, 2021. The copyright holder for this preprint (which was not certified by peer review) is the author/funder. All rights reserved. No reuse allowed without permission.

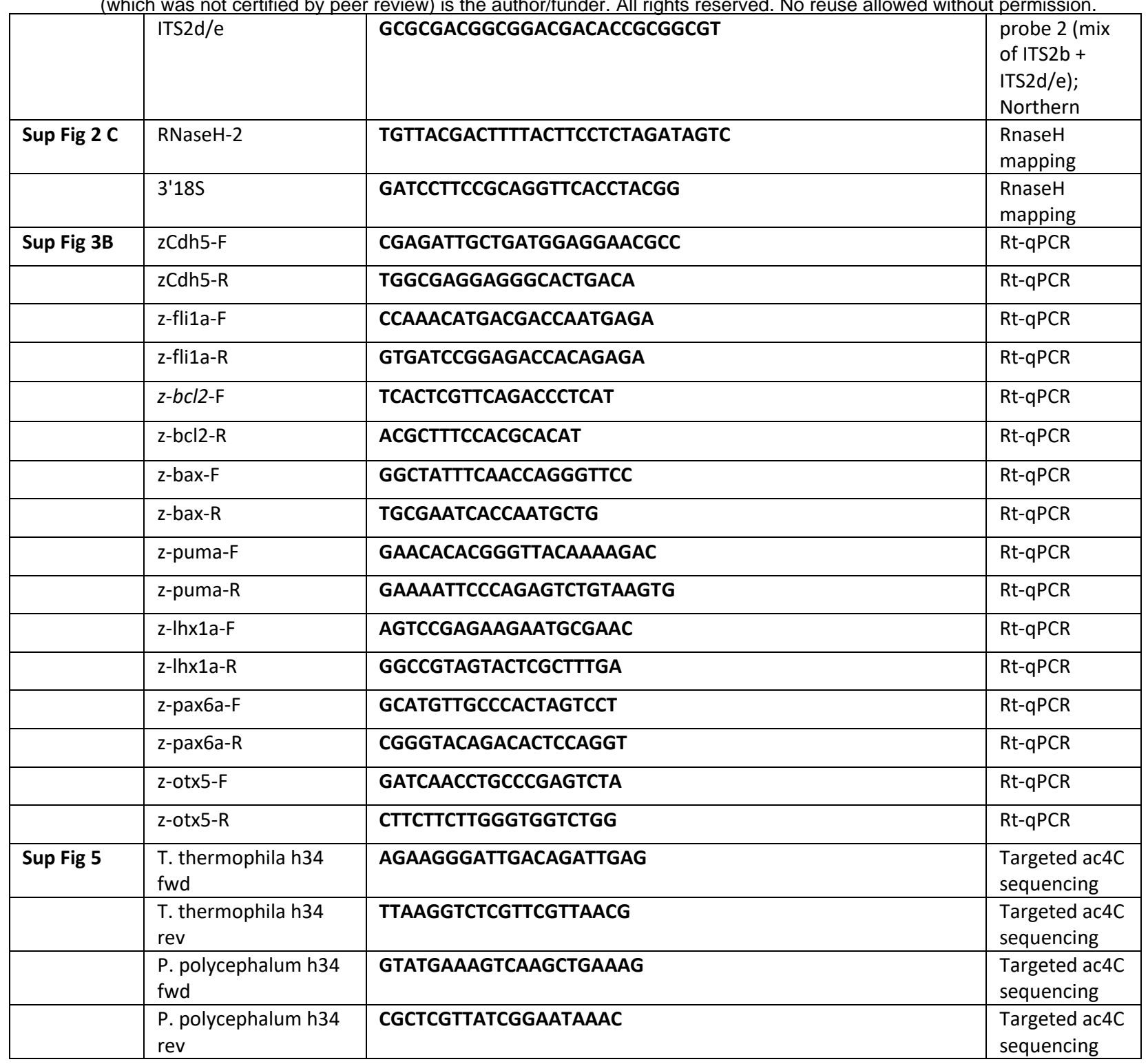

